\title{
TOTAL POSITIVITY, ABSORPTION PROBABILITIES AND APPLICATIONS(1)
}

\author{
BY \\ SAMUEL KARLIN
}

A function $K(x, y)$ of two real variables ranging over linearly ordered sets $X$ and $Y$ respectively is said to be totally positive of order $r\left(\mathrm{TP}_{r}\right)$ if for all $1 \leqq m \leqq r, x_{1}<x_{2}<\cdots<x_{m}, y_{1}<y_{2}<\cdots<y_{m}\left({ }^{2}\right)\left(x_{i} \in X, y_{j} \in Y\right)$, we have the inequalities

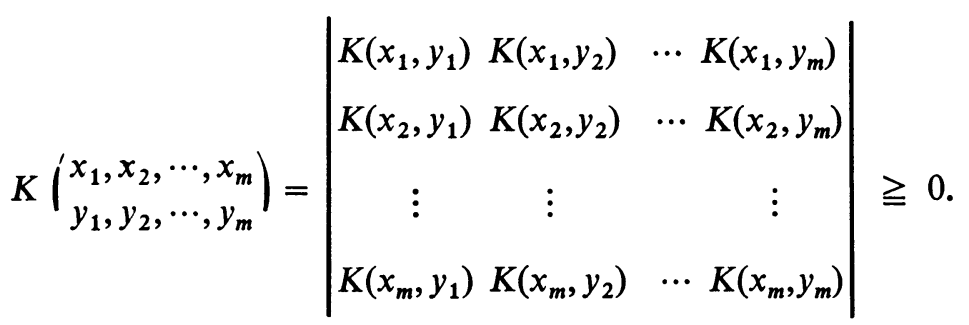

Typically, $X$ is an interval of the real line, or a countable set of discrete values on the real line such as the set of all integers or the set of non-negative integers; similarly for $Y$. When $X$ or $Y$ is a set of integers, we may use the term "sequence", rather than "function."

A related concept is that of sign regularity. A function $K(x, y)$ is sign regular of order $r$ if for every $x_{1}<x_{2}<\cdots<x_{m}, y_{1}<y_{2}<\cdots<y_{m}\left(x_{i} \in X ; y_{j} \in Y\right)$ and $1 \leqq m \leqq r$

$$
\operatorname{sign} K\left(\begin{array}{l}
x_{1}, x_{2}, \cdots, x_{m} \\
y_{1}, y_{2}, \cdots, y_{m}
\end{array}\right)=\varepsilon_{m}
$$

depends on $m$ alone. In the case where $\varepsilon_{m}=(-1)^{m(m-1) / 2}$ we then say that $K$ is " $R R_{r}$ ", where the letters stand for the abbreviation of sign reverse rule and suggest that $(0.2)$ would be positive except that the rows have been interchanged and appear in reverse order.

If a $\operatorname{TP}_{r}$ function $K(x, y)$ is a probability density in one of the variables, say $x$, with respect to a $\sigma$-finite measure $\mu(x)$, for each fixed value of $y$, then

Received by the editors October 30, 1962.

(1) This work was supported in part by the Office of Naval Research, Contract Nonr 225-28 at Stanford University and reproduction in whole or in part is permitted for any purpose of the United States Government.

(2) Unless stated explicitly to the the contrary, the determinants (0.1) always have the rows and columns ordered so that $x_{t}$ and $y_{j}$ are arranged by increasing values. 
$K(x, y)$ is said to be Pólya type of order $r\left(\mathrm{PT}_{r}\right)$. Every density is $\mathrm{PT}_{1}$, while the $\mathrm{PT}_{2}$ densities are those possessing a monotone likelihood ratio [25].

An important specialization occurs if a $\mathrm{PT}_{r}$ kernel may be written as a function $K(x-y)$ of the difference of $x$ and $y$ where $x$ and $y$ traverse the real line; $K(u)$ is then said to be a Pólya frequency density of order $r\left(\mathrm{PF}_{r}\right)$. If $x$ and $y$ range through the set of integers, $K(u)$ is said to be a Pólya frequency sequence of order $r$ which we abbreviate in the same way $\left(\mathrm{PF}_{r}\right)$. Finally, if the subscript $\infty$ is written in any of the definitions, then the property in question will be understood to hold for all values of $r$.

New totally positive functions are generated by means of the following composition law which can be viewed as a continuous version of the multiplication rule that evaluates subdeterminants in terms of those of the determinants of the same order of each factor.

LEMMA 0.1 . Let $X, Y$ and $Z$ represent linear sets on the real line. If

$$
M(x, z)=\int K(x, y) L(y, z) d \sigma(y)
$$

where $\sigma$ is a sigma-finite regular measure, then

$$
M\left(\begin{array}{l}
x_{1}, x_{2}, \cdots, x_{m} \\
z_{1}, z_{2}, \cdots, z_{m}
\end{array}\right)
$$

$$
=\int_{y_{1}<y_{2}<\ldots<y_{m}} \ldots \int K\left(\begin{array}{l}
x_{1}, x_{2}, \cdots, x_{m} \\
y_{1}, y_{2}, \cdots, y_{m}
\end{array}\right) L\left(\begin{array}{l}
y_{1}, y_{2}, \cdots, y_{m} \\
z_{1}, z_{2}, \cdots, z_{m}
\end{array}\right) d \sigma\left(y_{1}\right) d \sigma\left(y_{2}\right) \cdots d \sigma\left(y_{m}\right) .
$$

The proof appears in [30, p. 48, problem 68]. We shall exploit this formula rather extensively throughout the paper. For example, we immediately infer that if $K$ is $\mathrm{TP}_{r}$ and $L$ is $\mathrm{TP}_{s}$ then $M$ is $\mathrm{TP}_{\min (r, s)}$.

An important feature of totally positive functions is their variation diminishing property (abbreviated V.D.P.): Let $K(x, y)$ be $\mathrm{TP}_{r}$ and suppose $f(y)$ changes $\operatorname{sign}\left({ }^{3}\right) j \leqq r-1$ times, let $g(x)=\int K(x, y) f(y) d \mu(y)(d \mu(y)$ denotes a sigmafinite measure) be well defined such that the integral converges absolutely, then $g(x)$ changes sign at most $j$ times; moreover, if $g(x)$ actually changes sign $j$ times, then $g(x)$ must have the same arrangement of signs as the function $f(y)$ as $x$ and $y$ traverse their respective domains from left to right.

This property underlies many of the applications associated with totally positive kernels. The variation diminishing property is essentially equivalent to the inequalities (0.1), [9, Chapter 5], [33], [14].

(3) The number of sign changes, $V(f)$, of a real-valued function $f$ is $\sup V\left(f\left(x_{i}\right)\right)$, where $V\left(f\left(x_{i}\right)\right)$ denotes the number of sign changes of the sequence $f\left(x_{1}\right), f\left(x_{2}\right), \cdots, f\left(x_{m}\right)$ (zero values are discarded), with $x_{i}$ chosen arbitrarily from the domain of definition of $f$ and arranged so that $x_{1}<x_{2}<\cdots<x_{m} ; m$ is any positive integer. 
The theory of totally positive kernels has been extensively applied in several domains of mathematics, statistics, and mechanics. We indicate briefly some of the relevant background and literature of the subject. Pólya, Schur and Laguerre investigated in various forms the class of functions which can be approximated uniformly in every finite interval by polynomials having only real zeros. They proved that the only functions $K(u)$ which admit such approximations possess the property that $K(x-y)$ is $\mathrm{TP}_{\infty}$ : i.e., the function $K(u)$ is $\mathrm{PF}_{\infty}$.

Schoenberg in a series of remarkable papers has made an exhaustive study of the variation diminishing properties of totally positive kernels, where the variables $x$ and $y$ occur in translation form. (The paper [32] contains an exposition of some of his results up to the year 1953.) The theory of variation diminishing transformations developed for convolution kernels by Schoenberg was extended by this author to the general totally positive kernels in [13], [14], [25] and applied in characterizing best statistical procedures for a variety of decision problems.

Recently, Hirschman [11] has developed a class of variation diminishing transformations and representation formula for certain Bessel kernels and other kernels based on ultraspherical polynomials. His results comprise a nonhomogeneous extension of the theory of Schoenberg for certain classical functions which generalize the translation operation.

Hirschman and Widder [12] were interested in developing methods of inverting, by differential polynomial operators, certain integral transformations characterized by convolution kernels. This class of kernels is intimately related to the class of the Pólya frequency functions.

The theory of vibrations of certain types of mechanical systems (primarily coupled systems) depends on various aspects of the theory of totally positive functions [9].

Recently J. L. McGregor and this author [20] showed that the transition probability function $P(t, x, E)$ of a temporally homogeneous strong Markoff process $X(t)$ whose state space is the real line and which possesses a realization with almost all sample paths continuous is totally positive in the variables $x$ and $E$. Thus, if $x_{1}<x_{2}<\cdots<x_{m}$ and $E_{1}<E_{2}<\cdots<E_{m} \quad(E<F$ means that the points in $E$ all lie to the left of the points in $F$ ) then

$$
\operatorname{det} P\left(t, x_{i}, E_{j}\right) \geqq 0 \quad \text { for every } t>0 \text { and integer } m .
$$

The converse is also true; under suitable mild smoothness conditions the inequalities $(0.5)$ imply the existence of a realization of the process in which almost all sample paths are continuous. It follows from $(0.5)$ that whenever $P(t, x, E)$ has a continuous density function $p(t, x, y)$ then this is $\mathrm{TP}_{\infty}$ in the variables $x$ and $y$ for every $t>0$.

In this discussion continuity of path functions must be interpreted appropriately relative to the natural state space of the process, roughly speaking, continuity 
with respect to a suitable order topology. In particular, if the state space of the process is countable discrete then continuity of the path functions means that in every transition of the process the "position" of the particle (the state variable) changes to one of its neighboring states. Thus, discrete-state continuous path processes coincide with the so-called birth-and-death processes [17]. Consequently, for a birth-and-death stochastic process, the transition probability function $P_{i j}(t)$ (here, the state space is customarily identified with the non-negative integers) is totally positive in the variables $i$ and $j$ for every $t>0$.

In this paper we characterize new classes of totally positive and sign-regular kernels. These appear as absorption probabilities for stochastic processes whose one-step transition probability function is totally positive in the initial and final state variables. The totally positive kernels derived from these stochastic processes now involve variables one of which corresponds to time while the other is associated with the state of the process. The initial inspiration of this paper came from a result of F. Proschan [31] who was studying certain models of reliability. (See also [24].)

We now summarize partially the content and organization of this paper. In $\S 1$ we review some of the basic facts concerning $\mathrm{TP}_{r}$ functions to be used in our later developments. The material of this section is based largely on part of a forthcoming monograph by this author and consists of a survey of several alternative formulations of the concept of total positivity (see [15]). We offer only sketchy proofs here; a complete elaboration of the theory will appear in the above mentioned book. The results are set forth from the viewpoint of their pertinence to the discussions of the paper. Three successively stronger notions of total positivity are introduced and their relationships are described. Each of these definitions carries with it a corresponding strengthened version of the variation diminishing property.

In $\$ 2$ we commence the main developments of this paper, concentrating first on the case of discrete-time Markoff chains. The transition matrix is postulated to be totally positive in the state variables. The following two statements are typical of the results of this section. (Theorem 2.6) Let $\mathscr{P}$ be a temporally homogeneous $\mathrm{TP}_{r}$ Markoff chain (the transition probability matrix $P_{i j}$ is $\mathrm{TP}_{r}$ ) whose state space is the non-negative integers. Then the $n$-step transition function $P_{0 j}^{n}$ is $\mathrm{TP}_{r}$ in the variables $0 \leqq j<\infty$ and $n \geqq 0$. (Theorem 2.1) Let $\mathscr{P}$ be a $\mathrm{TP}_{r}$ Markoff chain. Let $F_{i, j_{0}}^{n}$ denote the probability that first passage into the set of states $\leqq j_{0}$ occurs at the $n$th transition when the initial state of the process is $i>j_{0}$. Then $F_{t, j_{0}}^{n}$ is $\mathrm{TP}_{r}$ in the variables $n \geqq 1$ and $i>j_{0}$.

These results are generalized in several different ways. By imposing further restrictions on the nature of the process, such as spatial homogeneity, non-negative drift, etc., we can display other sign-regular kernels constructed from various probability expressions natural to the process.

The results of $\$ 2$ are extended in $\$ 3$ to the case of discrete-time continuous- 
state Markoff processes on the real line. $\$ 4$ is devoted to the investigation of the analogs of the preceding results for the case of continuous-time temporally homogeneous Markoff chains whose transition matrix is denoted by $P_{i j}(t)$. Underlying the theorems of $\S 2$ was the basic stipulation that the transition matrix itself be $\mathrm{TP}_{r}$. In dealing with continuous time, we must require that $P_{i j}(t)$ is $\operatorname{TP}_{r}$ in the variables $i$ and $j$ for each $t>0$. Under this condition the process is necessarily of the birth-and-death type [22]. As a typical result (Theorem 4.1) we prove that the density function $q\left(t ; i, j_{0}\right)$ of the first passage time from state $i$ to state $j_{0}<i$ is $\mathrm{TP}_{r}$ in the variables $t>0$ and $i>j_{0}$.

In $\$ 5$ the theory is extended to the case of linear diffusion processes. These processes are characterized by the Strong-Markoff property and continuity of sample paths.

The analysis followed in $\S \S 4$ and 5 proceeds by discretizing the time scale. In this way we induce a discrete-time process of the type studied in $\S \S 2$ and 3 . The required first-passage probability functions are constructed which are sign regula $\mathrm{r}$ by virtue of the developments of these sections. A limiting procedure then leads to the corresponding conclusions for the continuous-time process.

In $\S 6$ we examine the relationship of the preceding results to the variation diminishing property. For example, we prove that if $\mathscr{P}$ is a symmetric temporally homogeneous $\mathrm{TP}_{r}$ Markoff chain (symmetry means that the transition matrix satisfies $P_{i j}=P_{-i-j}$ ) then

$$
V\left(\left\{\sum_{k=1}^{v} c_{k} P_{0 i}^{n_{k}}\right\}\right) \leqq 2 V\left(\left\{c_{k}\right\}\right), \quad v \leqq r
$$

where $V$ denotes the number of sign changes of the indicated sequence. The factor 2 in this inequality is essential. Some analogs and extensions are also given for the case of continuous time processes.

In $\$ 7$ a variety of composition laws are described from which we derive new totally positive kernels. The concept of subordination of stochastic processes plays a key role. An application to branching processes is indicated.

In $\S 8$ we set forth a series of illustrations and applications of the previous theorems. We start by treating some examples of generalized coin tossing where the probability of success may vary from trial to trial. Subsequently, some examples of random walks are discussed. Another example involves the distribution of the noncentral chi square. It is shown that this density is related to the first passage density function for the case of coin tossing where the waiting time between trials is random following an exponential distribution. It follows that this density is appropriately sign regular. As a consequence of our theory we also deduce the fact that this distribution is unimodal.

Some examples related to Brownian motion and general linear diffusion processes are discussed. 
In the midst of our studies of these examples we also present some applications to the theory of Tchebycheffian systems of functions. A sequence of functions $\phi_{0}(x), \phi_{1}(x), \phi_{2}(x), \cdots$ is said to constitute a Tchebycheff system abbreviated (T.S.) on $a<x<b$ (sometimes called Haar systems) if for any set of real constants $\left\{c_{k}\right\}$ not all zero, the function $\sum_{k=1}^{n} c_{k} \phi_{k}(x)=\phi(x)$ does not change sign more than $n-1$ times as $x$ traverses the interval $(a, b)$.

Let $\lambda_{0}, \lambda_{1}, \lambda_{2}, \cdots$ be arbitrary positive numbers and consider

$$
\phi_{k}(x)=\frac{1}{\left(\lambda_{0}+x\right)\left(\lambda_{1}+x\right) \cdots\left(\lambda_{k}+x\right)}, \quad k=0,1, \cdots \text {. }
$$

Then $\phi_{k}(x)$ constitutes a T.S. This result generalizes an example of Pólya-Szegö [30, problem 84$\rfloor$ and was deduced by invoking the total positivity property established for the first-passage probability function of an associated pure birth process.

Let $Q_{k}(x), k=0,1, \cdots$ represent a system of polynomials orthogonal with respect to a weight function concentrating on the non-negative axis. A consequence of Theorem 4.1 for birth-and-death processes is that $Q_{k}(-s), s>0$, constitutes a T.S. These examples are typical; other applications of the same kind are given. The examples of $\S 8$ were motivated by probability considerations. In $\$ 9$ we discuss some examples where the emphasis is on analytical methods. In this connection the extent of total positivity for various positive stable laws is investigated.

In $\$ 10$ we point out numerous qualitative properties of totally positive functions. We describe some typical results of this section. Consider a $\mathrm{TP}_{3}$ process on the non-negative real line. It is proved that the $n$ step transition density $P^{n}(0, x)$ is unimodal as a function of $n$ for fixed $x>0$. In the same vein, it is established that the density function of the first-passage time from position 0 to the set $[x, \infty)$ is a unimodal function. Similar conclusions are valid in the case of birth-anddeath and diffusion processes.

The interesting fact that the ergodic limits are approached monotonely emerges as a consequence of total positivity. In the case of $\mathrm{PF}_{r}$ functions $f(u)$ we can even estimate the rate at which $f^{\prime}(u)$ tends to zero at $\pm \infty$; moreover, $\mathrm{PF}_{\infty}$ functions may be characterized by moment inequalities and so on; for a discussion of these ideas, see [32].

Also in $\$ 10$ a strong ratio theorem is indicated for null recurrent $\mathrm{TP}_{2}$ Markoff chains whose state space is the non-negative integers. (Theorem 10.1) Let $\mathscr{P}$ be a $\mathrm{TP}_{2}$ temporally homogeneous irreducible recurrent Markoff chain whose state space is the non-negative integers. Then for any four prescribed states, $i, j, k, l$, $\lim _{n \rightarrow \infty}\left(P_{i j}^{n} / P_{k l}^{n}\right)$ exists, is finite and positive.

In the final section we study the so-called absolute value process associated with a symmetric Pólya frequency function. In closing this introduction, I wish 
to express my indebtedness to C. Stone and W. Studden for many valuable comments. Theorems 11.1 and 11.2 are due jointly to C. Stone and this writer.

1. Definitions and preliminaries. The monotonic character of a function $h(t)$ of a real variable can be expressed in various forms. We have the standard concept that $h(t)$ is increasing if $h\left(t_{1}\right) \leqq h\left(t_{2}\right)$ whenever $t_{1}<t_{2}$. A second notion concerns strict monotonicity and entails strict inequalities in the previous definition. It follows that if $h(t)$ is differentiable everywhere on an open interval $I$ of the real line, then $h^{\prime}(t) \geqq 0$ for $t \in I$ and equality may hold at certain values of $t$ even when $h(t)$ is strictly increasing. This suggests a third stronger concept. We say that $h(t)$ is strongly increasing if $h^{\prime}(t)>0$ for all $t$ in $I$.

Analogous to the case of monotonicity we formulate various definitions of total positivity with obvious containing relations amongst them. The first definition is that expressed in relation (0.1). Second, and analogous to the concept of strict monotonicity we say that $K(x, y)$ is strictly $\mathrm{TP}_{r}$ if strict inequality holds in $(0.1)$ (abbreviated STP $_{r}$ ). In order to state the analog of the derivative condition of the preceding paragraph we develop some preliminaries. We shall now assume that $X$ and $Y$ are open intervals of the real line and $K(x, y)$ belongs to $C^{r}$ (i.e., $K$ is $r$-fold continuously differentiable).

It follows by performing suitable manipulations on the rows and columns of (0.1) with reference to the mean value theorem, that for $x \in X, y \in Y$

$$
\left|\begin{array}{cccc}
K(x, y) & \frac{\partial K(x, y)}{\partial y} & \cdots & \frac{\partial^{m-1} K(x, y)}{\partial y^{m-1}} \\
\frac{\partial K(x, y)}{\partial x} & \frac{\partial^{2} K(x, y)}{\partial x \partial y} & \cdots & \frac{\partial^{m} K(x, y)}{\partial x \partial y^{m-1}} \\
\vdots & \\
\frac{\partial^{m-1} K(x, y)}{\partial x^{m-1}} & \frac{\partial^{m} K(x, y)}{\partial^{m-1} x \partial y} & \cdots & \frac{\partial^{2 m-2} K(x, y)}{\partial^{m-1} x \partial^{m-1} y}
\end{array}\right| \geqq 0
$$

(see [14] for a formal proof).

It is important to note that even if $K$ is $\mathrm{STP}_{r}$, the determinant (1.1) for special choices may be zero.

We now generalize the inequality (1.1). To this end, it is necessary to provide a special interpretation for the determinant

$$
K^{*}\left(\begin{array}{l}
x_{1}, x_{2}, \cdots, x_{m} \\
y_{1}, y_{2}, \cdots, y_{m}
\end{array}\right)
$$

where $x_{1} \leqq x_{2} \leqq \cdots \leqq x_{m}$ and $y_{1} \leqq y_{2} \leqq \cdots \leqq y_{m}$ and distinguished in that some 
of the $x$ 's or $y$ 's can be equal. (The asterisk sign on the $K$ shall frequently occur for purposes of emphasis when one or more of the $x$ 's or $y$ 's are equal and indicates that the special evaluation as described below is to be made.) Explicitly when there occurs a block of $r$ equal $x$ values, in forming the determinant $K(:::)$ the corresponding rows are determined by the successive derivatives, i.e., $K, \partial K / \partial x$, $\partial^{r-1} K / \partial x^{r-1}$. For examples, in the case of two equal $x$ values the first row associated with these values is formed in the standard way and consists of the elements $K\left(x, y_{1}\right), K\left(x, y_{2}\right), \cdots, K\left(x, y_{m}\right)$; the following row consists of the elements

$$
\frac{\partial K}{\partial x}\left(x, y_{1}\right), \frac{\partial K}{\partial x}\left(x, y_{2}\right), \cdots, \frac{\partial K}{\partial x}\left(x, y_{m}\right) \text {. }
$$

Blocks of equal $y$ values are treated analogously. In this case partial derivatives with respect to the $y$ variable occur wherever necessary. As a special case, we obtain that $K^{*}\left(\begin{array}{l}x, x, \ldots, x \\ y, y, \ldots, y\end{array}\right)$ denotes the determinant (1.1).

A slight modification of the method that leads from (0.1) to (1.1) also yields the result that if $K$ is $\mathrm{TP}_{r}$ then

$$
K^{*}\left(\begin{array}{l}
x_{1}, x_{2}, \cdots, x_{m} \\
y_{1}, y_{2}, \cdots, y_{m}
\end{array}\right) \geqq 0 \text { for }\left(\begin{array}{c}
x_{1} \leqq x_{2} \leqq \cdots \leqq x_{m} \\
y_{1} \leqq y_{2} \leqq \cdots \leqq y_{m}
\end{array}\right), 1 \leqq m \leqq r,
$$

$x_{i} \in X$ and $y_{j} \in Y$.

We are now ready to propose a definition of total positivity in terms of derivatives of $K$ which can be regarded as the counterpart of the concept of strong monotonicity. The function $K$ is said to be extended totally positive of order $r$ in the $x$ variable (abbreviated $\operatorname{ETP}_{r}(x)$ ) if

$$
K^{*}\left(\begin{array}{l}
x, x, \cdots, x \\
y_{1}, y_{2}, \cdots, y_{m}
\end{array}\right)>0 \text { for all } \quad \begin{aligned}
& x \in X \\
& y_{1}<y_{2}<\cdots<y_{m} ; \quad y_{j} \in Y .
\end{aligned}
$$

The emphasis is on strict inequality since the case of inequality with equalities always holds for $\operatorname{TP}_{r}$ functions. Similarly we may define the concept of $\operatorname{ETP}_{r}(y)$. Finally we say that $K$ is extended totally positive of order $r$ in both variables (written ETP, with no reference to either the $x$ or $y$ variable) if

$$
K^{*}\left(\begin{array}{l}
x, x, \cdots, x \\
y, y, \cdots, y
\end{array}\right)>0 \text { for } \quad \text { all } x \in X \text { and } y \in Y,
$$

the determinants being of size $m(1 \leqq m \leqq r)$. The definition of extended total positivity is meaningful only if $K$ is sufficiently continuously differentiable. This we will always assume is the case unless stated explicitly to the contrary.

An adapation of the arguments in [14, Theorem 1] proves that if $K$ is $\operatorname{ETP}_{r}(x)$ or $\operatorname{ETP}_{r}(y)$ then $K$ is $\operatorname{STP}_{r}$. By the same technique we can prove that if $K$ is $\operatorname{ETP}_{r}$ then 


$$
K^{*}\left(\begin{array}{l}
x_{1}, x_{2}, \cdots, x_{m} \\
y_{1}, y_{2}, \cdots, y_{m}
\end{array}\right)>0
$$

for arbitrary $x_{i}, y_{j}$ satisfying the conditions stated in (1.2), $m \leqq r$.

We have introduced four definitions of total positivity; namely $\mathrm{TP}_{r}, \mathrm{STP}_{r}$ $\operatorname{ETP}_{r}(x)$ or $\operatorname{ETP}_{r}(y)$ and $\operatorname{ETP}_{r}$, each of which implies the preceding property. All four definitions are useful in different contexts. In the general case these four levels of total positivity are inequivalent. However, in some special important cases the properties of $\mathrm{STP}_{r}$ and $\mathrm{ETP}_{r}$ are equivalent. This is so if $K(x, y)=f(x-y)$ where $f$ is $r$-fold continuously differentiable; the proof can be found in [15].

For later purposes we briefly indicate the extent of the variation diminishing property of the transformation

$$
g(x)=\int K(x, y) h(y) d \sigma(y)
$$

where $K(x, y)$ is assumed to be appropriately totally positive in one of the senses described above; $\sigma$ represents a sigma-finite measure. For ease of exposition we postulate that $h, g$ and $K$ occurring in (1.6) are sufficiently continuous so that all operations executed on the integral are justified.

The variation diminishing property (V.D.P.) in the case of $\mathrm{TP}_{r}$ functions was already stated in the introduction. If now we assume that $K$ is $\operatorname{ETP}(x)$ and that enough differentiations under the integral sign are permissible then the statement of the V.P.D. can be strengthened as follows. Let $V(h)$, as previously, denote the number of sign changes of the function $h$. Also, let $Z(g)$ designate the number of zeros, counting multiplicities, of the function $g$. Then if $K$ is $\operatorname{ETP}(x)$, and $g$ and $h$ are related according to (1.6), we have

$$
Z(g) \leqq V(h) .
$$

The concept of STP being intermediate between TP and $\operatorname{ETP}(x)$ possesses a variation diminishing property which is likewise intermediate between that stated in the introduction and (1.7). The proof of these assertions and further refinements will appear in [15]; they are not required for our present purposes.

It is much easier to operate with $\mathrm{STP}_{r}$ functions rather than with functions which are merely $\mathrm{TP}_{r}$. In this connection, we cite the fact that $\mathrm{TP}_{r}$ functions, under slight conditions, can be approximated by STP $r$ functions. We describe precisely the nature of this approximation (cf. [13]).

We say that a continuous function $K(x, y)$ dominated by exponential growth in $x$ and $y$ is regular $\mathrm{TP}_{r}$ for $-\infty<x, y<\infty$ if (i) $K(x, y)$ is $\mathrm{TP}_{r}$ on the real line, (ii) for any $m \leqq r$ and any prescribed set $x_{1}<x_{2}<\cdots<x_{m}$ there exists a set $y_{1}<y_{2}<\cdots<y_{m}$ which may depend on $\left(x_{1}, x_{2}, \cdots, x_{m}\right)$ such that the determinant $(0.1)$ formed from $x_{i}$ and $y_{j}$ is strictly positive or (ii') a symmetrical condition with $\left(y_{j}\right)$ prescribed holds. Define now 


$$
K_{\sigma}(x, y)=\frac{1}{\sqrt{ }(2 \pi) \sigma} \int_{-}^{\infty} \exp \left[-(x-u)^{2} / 2 \sigma^{2}\right] K(u, y) d u
$$

then, by virtue of $(0.4)$ and the regularity hypothesis we deduce that $K_{\sigma}(x, y)$ is $\mathrm{STP}_{r}$. Moreover, $K_{\sigma}(x, y)$ converges pointwise to $K(x, y)$ for every pair $-\infty<x, y<\infty$ as $\sigma \rightarrow 0$ and the approximations are established.

This same device can be frequently used in verifying the total positivity property (e.g., see §9, Lemma 9.1).

2. Total positivity and discrete-time Markoff chains. In this section we postulate total positivity of the underlying transition probability matrix and deduce total positivity for various other probability functions of the process. The most relevant are first passage probabilities into a specified set of states.

Consider a discrete-time Markoff process $\mathscr{P}$ whose state space is identified with the integers of the real line. The one-step transition probability matrix at the time $n$ is denoted by $P_{n}(i, j)$ where $-\infty<i, j<\infty$ and $n=1,2, \cdots$. Let the random variable $X(n)$ represent the position at time $n$ of a particle undergoing the process $\mathscr{P}$. The Markoff chain $\mathscr{P}$ is said to be temporally homogeneous (homogeneous in time) if $P_{n}(i, j)=P(i, j)$ is independent of $n$, and to be spatially homogeneous (homogeneous in space) if $P_{n}(i, j)=P_{n}(0, j-i)$ for all $n \geqq 1$ and $-\infty<i, j<\infty$. In the latter case $X(n)$, where $X(0)=0$, represents a sum of independent, not necessarily identically distributed random variables $\xi_{m}(m=1,2, \cdots, n)$ with density functions $\operatorname{Pr}\left\{\xi_{m}=i\right\}=P_{m}(0, i)$. The process $\mathscr{P}$ is said to have non-negative (positive) drift if $P_{n}(i, j)=0$ for $j<i(j \leqq i)$. The process $\mathscr{P}$ is called symmetric if $P_{n}(i, j)=P_{n}(-i,-j)$ for all $-\infty<i, j<\infty$ and $n \geqq 1$.

The process $\mathscr{P}$ is said to be totally positive of order $r$ (abbreviated $\mathrm{TP}_{r}$ ) if for each $n$ the matrix $P_{n}(i, j)$ is $\mathrm{TP}_{r}$ with respect to the discrete variables $i$ and $j$. A matrix $A$ is said to have the property of the sign reverse rule (abbreviated $\left.\mathrm{RR}_{r}\right)$ if all $v \times v$ sub-determinants of $A$ have the sign $(-1)^{v(v-1) / 2}$, where $1 \leqq v \leqq r$. Clearly, $P(i, j)$ is $\mathrm{RR}_{r}$ if and only if $P(-i, j)$ is $\mathrm{TP}_{r}$. Finally, if $A$ and $B$ are sets of integers, we write $A \leqq B$ if $i \in A, j \in B$ implies $i \leqq j$ and $A<B$ if $i \in A$ $j \in B$ implies $i<j$.

We can now proceed to the main theorems of this section.

THEOREM 2.1. Let $\mathscr{P}$ be a $\mathrm{TP}_{r}$ Markoff chain (not necessarily temporally homogeneous), and let $B$ and $C$ be nonvoid sets of states. Define

$$
Q_{B}(n, i, C)=Q(n, i)=\operatorname{Pr}\{X(v) \in B \text { for } 1 \leqq v \leqq n-1, X(n) \in C \mid X(0)=i\}
$$

for $-\infty<i<\infty$ and $n \geqq 1$. (We suppress the dependence on $B$ and $C$ when no ambiguities can arise $\left({ }^{4}\right)$.)

(4) Henceforth such simplifications of notation will be done without further explanations. 
(i) If $B \leqq C$ then $Q(n, i)$ is $\mathrm{RR}_{r}$.

(ii) If $B \geqq C$, then $Q(n, i)$ is $\mathrm{TP}_{r}$.

(iii) If $C$ is a set consisting of one element $\{c\}$ and $\{c\} \geqq B$, then (i) holds for $n \geqq 0$ and $-\infty<i<c$, where we define $Q(0, i)=\delta_{i c}$ ( $\delta$ denotes the standard Kronecker delta function). Similarly, if $C=\{c\}$ and $\{c\} \leqq B$, then (ii) holds for $n \geqq 0$ and $c \leqq i<\infty\left({ }^{5}\right)$.

RemarK. If we had defined $Q(0, i) \equiv 0$, (i) would trivially extend to include $n=0$. However, such a prescription is not convenient for later applications.

Proof. (a) Let

$$
Q^{(2)}(n, i)=\operatorname{Pr}\{X(v) \in B \text { for } 2 \leqq v \leqq n, X(n+1) \in C \mid X(1)=i\} .
$$

Observe that $Q^{(2)}$ is defined like $Q$ in (2.1) except that time is advanced one unit. A simple enumeration of events gives the renewal relation

$$
Q(n, i)=\sum_{k \in B} P_{1}(i, k) Q^{(2)}(n-1, k)
$$

It follows from (2.2) by virtue of (0.4) that

$$
\begin{aligned}
& Q\left(\begin{array}{c}
n_{1}, \cdots, n_{v} \\
i_{1}, \cdots, i_{v}
\end{array}\right) \\
& \quad=\sum_{\substack{k_{1}<k_{2}<\ldots<k_{v} \\
k_{1}, k_{2}, \ldots \\
k_{v} \in B}} P_{1}\left(\begin{array}{l}
i_{1}, i_{2}, \cdots, i_{v} \\
k_{1}, k_{2}, \cdots, k_{v}
\end{array}\right) Q^{(2)}\left(\begin{array}{c}
n_{1}-1, n_{2}-1, \cdots, n_{v}-1 \\
k_{1}, \quad k_{2}, \cdots, \quad k_{v}
\end{array}\right),
\end{aligned}
$$

provided $2 \leqq n_{1}<n_{2}<\cdots<n_{v}$ and $i_{1}<i_{2}<\cdots<i_{v}$ and $v \geqq 1$. We now establish the formula

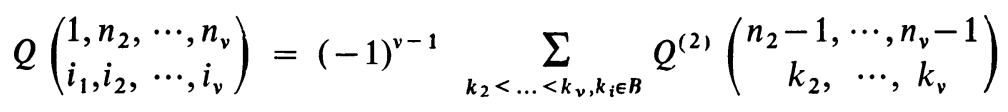

$$
\times \sum_{l \in C} P_{1}\left(\begin{array}{l}
i_{1}, \cdots, i_{v-1}, i_{v} \\
k_{2}, \cdots, k_{v}, l
\end{array}\right)
$$

(if $v=1$, the first summation is missing). To this end, we expand the left-hand side of (2.4) along the first row, obtaining

$$
Q\left(\begin{array}{l}
1, n_{2}, \cdots, n_{v} \\
i_{1}, i_{2}, \cdots, i_{v}
\end{array}\right)=\sum_{s=1}^{v}(-1)^{s+1} Q\left(1, i_{s}\right) Q\left(\begin{array}{l}
n_{2}, \cdots, \cdots, \cdots, \cdots, n_{v} \\
i_{1}, \cdots, i_{s-1}, i_{s+1}, \cdots, i_{v}
\end{array}\right) .
$$

(5) We concentrate throughout on the ordinary sign regularity property leaving the case of strict sign regularity to the reader. 
Using the fact

$$
Q\left(1, i_{v}\right)=\sum_{l \in C} P_{1}\left(i_{v}, l\right)
$$

and substituting (2.3) into the right-hand side of (2.5), we obtain

$$
\begin{array}{r}
\sum_{s=1}^{v}(-1)^{s+1} \sum_{l \in C} P_{1}\left(i_{s}, l\right) \sum_{k_{2}<\ldots<k_{v} ; k_{i} \in B} P_{1}\left(\begin{array}{c}
i_{1}, \cdots, i_{s-1}, i_{s+1}, \cdots, i_{v} \\
k_{2}, \cdots, \cdots, \cdots, \cdots, k_{v}
\end{array}\right) \\
\times Q^{(2)}\left(\begin{array}{c}
n_{2}-1, \cdots, n_{v}-1 \\
k_{2}, \cdots, k_{v}
\end{array}\right) .
\end{array}
$$

This simplifies to

$$
\begin{gathered}
\sum_{k_{2}<\ldots<k_{v}: k_{i} \in B} Q^{(2)}\left(\begin{array}{c}
n_{2}-1, \cdots, n_{v}-1 \\
k_{2}, \cdots, k_{v}
\end{array}\right) \\
\quad \times \sum_{1 \leqq s \leqq v ; l \in C}(-1)^{s+1} P_{1}\left(i_{s}, l\right) P_{1}\left(\begin{array}{c}
i_{1}, \cdots, i_{s-1}, i_{s+1}, \cdots, i_{v} \\
k_{2}, \cdots, \cdots, \cdots, \cdots, k_{v}
\end{array}\right) \\
=\sum_{k_{2}<\ldots<k_{v} ; k_{i} \in B} Q^{(2)}\left(\begin{array}{c}
n_{2}-1, \cdots, n_{v}-1 \\
k_{2}, \cdots, k_{v}
\end{array}\right) \sum_{l \in C} P_{1}\left(\begin{array}{c}
i_{1} \cdots, i_{v} \\
l, k_{2}, \cdots, k_{v}
\end{array}\right) .
\end{gathered}
$$

The identity (2.4) is derived by interchanging the columns in

$$
P_{1}\left(\begin{array}{l}
i_{1}, \cdots, i_{v-1}, i_{v} \\
l, k_{2}, \cdots, k_{v}
\end{array}\right)
$$

With the aid of (2.3) and (2.4) we finish the proof of the theorem by employing mathematical induction twice, first with respect to $v$ and then with respect to $n_{1}$. To begin with, note that since $Q(n, i)$ is a probability, it is trivially $\mathbf{R R}_{1}$; indeed, non-negative. Next assume that the theorem holds for all Markoff processes $\mathscr{P}$ which are $\mathrm{TP}_{r}$, that is, the determinants

$$
Q\left(\begin{array}{l}
n_{1}, n_{2}, \cdots, n_{v-1} \\
i_{1}, i_{2}, \cdots, i_{v-1}
\end{array}\right)
$$

have a fixed sign depending only on the size of the determinant. We now prove that the theorem holds for determinants of size $v \times v$ for all Markoff chains $\mathscr{P}$ which are $\mathrm{TP}_{r}$. In fact, it follows from (2.4), the induction assumption on $v$, and the hypothesis of the theorem that for $n_{1}=1$

$$
\operatorname{sign} Q\left(\begin{array}{l}
1, n_{2}, \cdots, n_{v} \\
i_{1}, i_{2}, \cdots, i_{v}
\end{array}\right)=(-1)^{v-1}(-1)^{(v-1)(v-2) / 2}=(-1)^{v(v-1) / 2} .
$$


From (2.3) and (2.6), coupled with another induction procedure on $n_{1}$, we obtain

$$
\operatorname{sign} Q\left(\begin{array}{c}
n_{1}, n_{2}, \cdots, n_{v} \\
i_{1}, i_{2}, \cdots, i_{v}
\end{array}\right)=(-1)^{v(v-1) / 2} .
$$

Hence $Q(n, i)$ is $\mathrm{SRR}_{v}$, and the induction step is complete.

(ii) The proof is similar to that of (i).

(iii) Here,

$$
Q\left(\begin{array}{l}
0, n_{2}, \cdots, n_{v} \\
i_{1}, i_{2}, \cdots, i_{v}
\end{array}\right)=0 \quad\left(0<n_{2}<n_{3}<\cdots<n_{v} ; i_{1}<i_{2}<\cdots<i_{v}\right)
$$

unless $i_{v}=c$, in which case

$$
Q\left(\begin{array}{l}
0, n_{2}, \cdots, n_{v} \\
i_{1}, i_{2}, \cdots, i_{v}
\end{array}\right)=(-1)^{v-1} Q\left(\begin{array}{c}
n_{2}, \cdots, n_{v} \\
i_{2}, \cdots, i_{v}
\end{array}\right)
$$

and the sign of the second factor is determined as in part (i). It follows that

$$
(-1)^{v(v-1) ! 2} Q\left(\begin{array}{l}
0, n_{2}, \cdots, n_{v} \\
i_{1}, i_{2}, \cdots, i_{v}
\end{array}\right) \geqq 0,
$$

as it should be. The rest of the proof is unchanged

EXAMPLE. We identify $B$ with the set of states to the left of $b$ and $C$ with all the states to the right of $b$ and including $b$. Under the conditions of the theorem, we conclude that

$$
Q(n, i)=\operatorname{Pr}\{X(v)<b \text { for } 1 \leqq v \leqq n-1, X(n) \geqq b \mid X(0)=i\}
$$

is $\mathrm{RR}_{r}$ for $-\infty<i<\infty$ and $n \geqq 1$.

The next theorem shows that for Markoff chains that are temporally homogeneous and $\mathrm{TP}_{r}$, the first-passage probability function defined in (2.8) below is $\mathrm{TP}_{r}$ in the variables consisting of the final state and time. The reader should contrast this statement with that of Theorem 2.1, in which we are concerned with the initial-state variable rather than the final-state variable.

THEOREM 2.2. Let $\mathscr{P}$ be a temporally homogeneous $\mathrm{TP}_{r}$ Markoff chain, and let $B$ be a nonvoid set of states and a fixed state $a$ such that $a \leqq B$. Then

$$
Q(n, j)=\operatorname{Pr}\{X(v) \in B \text { for } 1 \leqq v \leqq n-1, X(n)=j \mid X(0)=a\}\left({ }^{6}\right)
$$

is $\mathrm{TP}_{r}$ for $-\infty<j<\infty$ and $n \geqq 1$. If we define $Q(0, j)=\delta_{a j}$, then (2.8) is TP, for $n \geqq 0$ and $a \leqq j<\infty$.

(6) We use frequently the common symbol $Q(n, j)$ to represent different probability expressions for which we investigate the property of sign regularity. This should cause no difficulty since the particular quantity at hand is always amply defined. 
The proof parallels that of Theorem 2.1. We use the renewal relation

$$
Q(n, j)=\sum_{k \in B} Q(n-1, k) P(k, j)
$$

in place of (2.2); the other details are the same.

Note that the factor $P(k, j)$ in (2.9) is independent of $n$, by virtue of the hypothesis of the theorem. This is decisive, since Theorem 2.2 is generally not valid if $\mathscr{P}$ is not assumed to be temporally homogeneous; a counterexample is given following Theorem 2.6.

Our next theorem interprets Theorem 2.2 for spatially homogeneous Markoff chains.

THEOREM 2.3. Let $\mathscr{P}$ be a spatially homogeneous $\mathrm{TP}_{r}$ Markoff chain. Suppose $B$ and $C$ are nonvoid sets.

(i) If $B \leqq C$, then

is $\mathrm{TP}_{r}$ for $-\infty<j<\infty$ and $n \geqq 1$. If $C$ consists of a single element $\{c\} \geqq B$, then (2.10) is $\mathrm{TP}_{r}$ for $j \geqq a-c$ and $n \geqq 0$, where $Q(0, j)=\delta_{a-c, j}$.

(ii) If $B \geqq C$, then (2.10) is $\mathrm{RR}_{r}$ for $-\infty<j<\infty$ and $n \geqq 1$; and if $C$ consists of a single element $\{c\},(2.10)$ is $\mathrm{R}_{r}$ for $j \leqq a-c$ and $n \geqq 0$.

Proof. Since $\mathscr{P}$ is homogeneous in space, it follows that

$$
\begin{aligned}
R(n,-j) & =\operatorname{Pr}\{X(v) \in B-j \text { for } 1 \leqq v \leqq n-1, X(n) \in C-j \mid X(0)=a\} \\
& =\operatorname{Pr}\{X(v) \in B-a \text { for } 1 \leqq v \leqq n-1, X(n) \in C-a \mid X(0)=j\} .
\end{aligned}
$$

The conditions of the theorem imply that $B-a \leqq C-a$, and we may apply Theorem 2.1. In particular, we infer that $R(n,-j)$ is $\operatorname{SRR}_{r}$ for $-\infty<j<\infty$ and $n \geqq 1$. Hence $R(n, j)=Q(n, j)$ is $\mathrm{TP}_{r}$, as was to be shown. The final statement follows as in Theorem 2.1.

EXAMPLE. Under the conditions of Theorem 2.3 we deduce that

$$
Q(n, j)=\operatorname{Pr}\{X(v) \leqq j-1 \text { for } 1 \leqq v \leqq n-1, X(n) \geqq j \mid X(0)=a\}
$$

is $\mathrm{TP}_{r}$ for $-\infty<j<\infty$ and $n \geqq 1$.

Following Theorem 2.6 we give an example to show that (2.11) does not generally hold when $\mathscr{P}$ is not spatially homogeneous.

We now list results of the above sort for the case in which $\mathscr{P}$ is a $\mathrm{TP}_{r}$ Markoff chain with non-negative drift. The reader may derive the corresponding results for the case in which $\mathscr{P}$ is a $\mathrm{TP}_{\boldsymbol{r}}$ Markoff chain of nonpositive drift. for $C+j$. 
THEOREM 2.4. Let $\mathscr{P}$ be a $\mathrm{TP}_{r}$ Markoff chain (not necessarily temporally homogeneous) with non-negative drift. Then

$$
Q(n, i)=\operatorname{Pr}\{X(n)=b \mid X(0)=i\} \text { is } \mathrm{R}_{r} \text { for } n \geqq 0 \text { and }-\infty<i<\infty
$$

and

$$
\begin{aligned}
Q(n, i)=\operatorname{Pr}\{X(v)<b \text { for } 1 \leqq v \leqq & n-1, X(n)=b \mid X(0)=i\} \\
& \text { is } \mathbf{R}_{r} \text { for } n \geqq 0 \text { and }-\infty<i<\infty
\end{aligned}
$$

(the usual delta function convention holds for $n=0$ ).

Proof. This theorem follows from Theorem 2.1 by suitable specialization. In order to deduce (2.12) we take $B$ as the set of states lying to the left of $b$ including $b$ and $C$ as the set consisting of the single state $\{b\}$. Since the process has nonnegative drift, the quantity in (2.12) reduces to the probability function defined in (2.1), and the desired conclusion is obtained by applying Theorem 2.1. In a similar manner we obtain (2.13).

THEOREM 2.5. Let $\mathscr{P}$ be a spatially homogeneous $\mathrm{TP}_{r}$ Markoff chain (not necessarily temporally homogeneous) with non-negative drift. Then

$$
\begin{array}{r}
Q(n, j)=\operatorname{Pr}\{X(n)=j \mid X(0)=a\} \text { is } \operatorname{TP}_{r} \text { for } a \leqq j<\infty \text { and } n \geqq 0, \\
Q(n, j)=\operatorname{Pr}\{x(v)<j \text { for } 1 \leqq v \leqq n-1, X(n)=j \mid X(0)=a\} \\
\quad \text { is } \operatorname{TP}_{r} \text { for } a \leqq j \leqq \infty \text { and } n \geqq 0 .
\end{array}
$$

In each case, $Q(0, j)=\delta_{a j}$.

Proof. In Theorem 2.3 we take $B$ as the set of all nonpositive states and $C$ as the set consisting of the single state $\{0\}$. Invoking Theorem 2.3, we deduce the validity of (2.14). The validity of (2.15) is deduced in a similar manner by identifying $B$ with the set of all negative states and $C$ with $\{0\}$.

Our next two propositions are in the same vein as the preceding theorems.

THEOREM 2.6. Let $\mathscr{P}$ be a temporally homogeneous $\mathrm{TP}_{r}$ Markoff chain whose state space is the non-negative integers. Then

$$
\begin{aligned}
& Q(n, j)=\operatorname{Pr}\{X(n)=j \mid X(0)=0\}=P^{n}(0, j) \text { is } \mathrm{TP}_{r} \text { for } 0 \leqq j<\infty \text { and } n \geqq 0 . \\
& Q(n, j)=\operatorname{Pr}\{X(n)>j \mid X(0)=0\} \text { is } \mathrm{TP}_{r} \text { for } 0 \leqq j<\infty \text { and } n \geqq 0, \\
& Q(n, j)=\operatorname{Pr}\{X(n) \leqq j \mid X(0)=0\} \text { is } \operatorname{TP}_{r} \text { for } 0 \leqq j<\infty \text { and } n \geqq 0 .
\end{aligned}
$$




$$
\begin{array}{r}
Q(n, j)=\operatorname{Pr}\{X(n)=0 \mid X(0)=j\}=P^{n}(j, 0) \text { then } Q(n, j) \text { is } \operatorname{TP}_{r} \text { for } \\
0 \leqq j<\infty \text { and } n \geqq 0 .
\end{array}
$$

In (2.16), (2.17) and (2.18), we define $Q(0, j) \equiv \delta_{0, j}$.

Proof. The proof of (2.16) parallels that of Theorem 2.1, with the ChapmanKolmogoroff relation

$$
Q(n, j)=\sum_{k=0}^{\infty} Q(n-1, k) P(k, j),
$$

replacing (2.2) in the line of argument. The proof of (2.18) uses the recursion relation

$$
Q(n, j)=\sum_{k=0}^{\infty} P(j, k) Q(n-1, k)
$$

We omit the details.

The verification of the first half of (2.17) rests on the formula

$$
Q(n, j)=\sum_{k=j+1}^{\infty} P^{n}(0, k)=\sum_{k=0}^{\infty} L(k, j) P^{n}(0, k)
$$

where

$$
L(k, j)= \begin{cases}1, & k>j \\ 0, & k \leqq j\end{cases}
$$

A direct computation shows that

$$
L\left(\begin{array}{c}
k_{1}, k_{2}, \cdots, k_{v} \\
j, j_{2}, \cdots, j_{v}
\end{array}\right) \geqq 0 \text { for } 0 \leqq\left\{\begin{array}{c}
k_{1}<k_{2}<\cdots<k_{v}, \\
j_{1}<j_{2}<\cdots<j_{v} .
\end{array}\right.
$$

By applying (0.4) to (2.19) and using (2.16), we establish (2.17). The proof of the second half of (2.17) is similar.

We may extract a general principle from the above argument as follows: Let $R(x, y)$ denote any sign-regular kernel in the variables $x, y$ with $x$ and $y$ restricted to the intervals $\alpha \leqq x \leqq \beta, \gamma \leqq y \leqq \delta$, respectively. Then all cumulatives of the form

$$
\begin{array}{ll}
R_{1}(x, y)=\int_{a}^{x} R(\xi, y) d \mu(\xi), & R_{2}(x, y)=\int_{x}^{\beta} R(\xi, y) d \mu(\xi), \\
R_{3}(x, y)=\int_{y}^{y} R(x, \eta) d \mu(\eta), & R_{4}(x, y)=\int_{y}^{\delta} R(x, \eta) d \mu(\eta),
\end{array}
$$

where $d \mu$ is a non-negative sigma-finite measure, are sign regular in the same sense as $R(x, y)$. The argument employs the same ideas used in verifying (2.17). 
Note that the functions given in (2.17) arise from (2.16) as cumulants.

COROLlaRY 2.1. Let $\mathscr{P}$ be a temporally homogeneous $\mathrm{TP}_{r}$ Markoff chain with non-negative drift. Then

$$
Q(n, j)=\operatorname{Pr}\{X(n)=j \mid X(0)=a\} \text { is } \operatorname{TP}_{r} \text { for } a \leqq j<\infty \text { and } n \geqq 0
$$

and

$$
Q(n, j)=\operatorname{Pr}\{X(n) \geqq j \mid X(0)=a\} \text { is } \operatorname{TP}_{r} \text { for } a \leqq j \leqq \infty \text { and } n \geqq 0 .
$$

In both cases, $Q(0, j)=\delta_{a j}$.

This result is a consequence of Theorem 2.6. Note that without any loss in generality we can view the process as taking place on the set of states $\{a, a+1, \cdots\}$. The state $a$ behaves like the zero state and Theorem 2.6 is in force.

We conclude this line of results with several remarks.

(i) In the case that $\mathscr{P}$ is a temporally homogeneous $\mathrm{TP}_{\boldsymbol{r}}$ Markoff chain with strictly positive drift (i.e., $p_{i j}=0$ for $j \leqq i$ ) then clearly $Q(n, j)$ as defined in $(2.20)$ coincides with the first-passage probability function

$$
F(n, j)=\operatorname{Pr}\{X(n)=j, X(v)<j \text { for } 1 \leqq v \leqq n-1 \mid X(0)=a\}
$$

and so $F(n, j)$ is $\mathrm{TP}_{r}$ for $j>a$ and $n \geqq 1$.

(ii) Another case for which we may prove that the function $F(n, j)$ is $\mathrm{TP}_{r}$ for $j>a$ and $n \geqq 1$ is when $\mathscr{P}$ has the form $P_{i i}=1-p_{i}, P_{i, i+1}=p_{i}\left(0<p_{i}<1\right)$. The proof depends on the relation

$$
F(n, j)=P_{a, j-1}^{n-1} p_{j-1}, \quad j \geqq a+1,
$$

and the desired inference follows by virtue of Corollary 2.1 .

(iii) We can prove generally that $F(n, j)$ is $\mathrm{TP}_{2}$, whenever $\mathscr{P}$ is a $\mathrm{TP}_{2}$ Markoff chain with a non-negative drift. Indeed,

where

$$
F(n, j)=\sum_{k=0}^{j-1} P_{a k}^{n-1} P_{k j}=\sum_{k=0}^{\infty} P_{a k}^{n-1} R_{k j}
$$

$$
R_{k j}= \begin{cases}P_{k j}, & k<j \\ 0, & \text { otherwise }\end{cases}
$$

Using this representation the arguments of Theorem 2.1 apply.

(iv) If $\mathscr{P}$ is a $\mathrm{TP}_{r+1}$ temporally homogeneous Markoff chain with non-negative drift in which $P_{i, i}=p>0$ is independent of $i \geqq 0$ and $P_{i, j}>0$ for all $j>i$ then $F(n, j)$ is $\mathrm{STP}_{r}$. To prove this we observe in this case that

$$
F(n, j)=P_{a j}^{n}-p P_{a j}^{n-1} \text {. }
$$

Now, by Theorem 2.5 


$$
Q\left(\begin{array}{ll}
n, n+1, & \cdots, n+v \\
a, j_{1}, & \cdots, j_{v}
\end{array}\right)>0,
$$

for $a<j_{1}<j_{2}<\cdots<j_{v}$ where $Q(n, j)=P_{a j}^{n}$. The emphasis is on strict inequality as may be ascertained by careful scrutiny of the proof of Theorem 2.5.

Elementary operations on the rows leads to the identity

$$
Q\left(\begin{array}{ll}
n, n+1, & \cdots, n+v \\
a, j_{1}, & \cdots, j_{v}
\end{array}\right)=p^{n} F\left(\begin{array}{l}
n+1, n+2, \cdots, n+v \\
j_{1}, j_{2},
\end{array}\right) .
$$

Thus we conclude that

$$
F\left(\begin{array}{l}
n+1, n+2, \cdots, n+v \\
j_{1}, \quad j_{2}, \quad \cdots, j_{v}
\end{array}\right)>0 \quad \text { for } a<j_{1}<j_{2}<\cdots<j_{v}
$$

and $n \geqq 0$. It suffices to apply Fekete's lemma [9,Chapter 5] and it follows that $F(n, j)$ is $\mathrm{STP}_{r}$ as asserted.

The foregoing remarks give evidence of the conjecture that $F(n, j)$ is always $\mathrm{TP}_{r}$ whenever $\mathscr{P}$ is a temporally homogeneous $\mathrm{TP}_{r}$ Markoff chain with nonnegative drift.

CountereXamples. Theorem 2.6 and Corollary 2.1 are false if we remove the hypothesis that $\mathscr{P}$ is temporally homogeneous. For example, statement (2.20) does not hold in the following circumstances: Set $P_{n}(i, j)=0$ except when $j=i$ or $j=i+1$, in which case set $P_{n}(i, i)=q_{i}^{(n)}$ and $P_{n}(i, i+1)=p_{i}^{(n)}$. Such a transition probability matrix is clearly $\mathrm{TP}_{\infty}$. Moreover, with the $Q(n, j)$ defined as in (2.20), with $a=0$, we obtain

$$
Q\left(\begin{array}{ll}
1 & 2 \\
0 & 1
\end{array}\right)=\left|\begin{array}{cc}
q_{0}^{(1)} & p_{0}^{(1)} \\
q_{0}^{(1)} q_{0}^{(2)} & q_{0}^{(1)} p_{0}^{(2)}+p_{0}^{(1)} q_{1}^{(2)}
\end{array}\right|
$$

which can be made negative or positive by appropriate choice of $q_{0}^{(1)}, p_{0}^{(1)}, q_{0}^{(2)}$, $p_{0}^{(2)}, q_{1}^{(2)}$, the only restrictions being $q_{i}^{(n)}+p_{i}^{(n)}=1$ for all $i, n$ and $0<p_{i}^{(n)}<1$.

Theorem 2.6 is also generally false if $X(0)=a$, where $a>0$. As an example, consider

$$
P_{n}(i, j)=\left\{\begin{array}{ll}
\rho^{j-i+1}(1-\rho) & j=i-1, i, i+1, \cdots \\
0 & j<i-1
\end{array} \quad i \geqq 1\right.
$$

and $P_{n}(0, j)=\rho^{j}(1-\rho)$ for $j=0,1,2, \cdots$. It is a simple matter to verify that $P_{n}(i, j)$ is $\mathrm{TP}_{\infty}$, and a direct calculation shows that

$$
Q\left(\begin{array}{ll}
1, & 2 \\
0, & 1
\end{array}\right)<0, \text { and } Q\left(\begin{array}{ll}
1, & 2 \\
1, & 2
\end{array}\right)>0
$$

where

$$
Q(n, j)=\operatorname{Pr}\{X(n)=j \mid X(0)=2\} .
$$


We now indicate a simple example which shows that the conclusion of (2.11) is violated in the case that the process $\mathscr{P}$ is not spatially homogeneous. Indeed, we define the transition matrix over the set of non-negative states as $p_{00}=7 / 8$, $p_{01}=1 / 8 ; p_{10}=3 / 4, p_{11}=1 / 8, p_{12}=1 / 8 ; p_{i i}=1 / 8, p_{i, i+1}=7 / 8$ for $i \geqq 2$. It is trivial to verify that $\mathscr{P}$ is $\mathrm{TP}_{\infty}$. Consider $Q(n, j)$ defined as in (2.11) with $a=1$. Then a direct calculation gives $Q\left(\begin{array}{l}1,2 \\ 1,2\end{array}\right)<0$ and $Q\left(\begin{array}{l}1,2 \\ 2,3\end{array}\right)>0$. This shows that $Q(n, j)$ is not sign regular.

Time translation kernels. In the remainder of this section we derive several determinant inequalities that are valid for the functions $L(n, m)=Q(n+m, i)$, with $i$ fixed where $Q$ denotes one of the preceding probability expressions.

THEOREM 2.7. (i) Under the conditions of Theorem 2.6, the function $L(n, m)=Q(n+m, 0)$, where $Q$ is as defined in (2.16), is $\mathrm{TP}_{r}$ for $n, m \geqq 0$.

(ii) Under the conditions of Corollary 2.1, the function $L(n, m)=Q(n+m, j)$, where now $Q$ is as defined in (2.20), is $\mathrm{R}_{r}$ for $n, m \geqq 0$.

(iii) Let $\mathscr{P}$ be a temporally homogeneous $\mathrm{TP}_{r}$ Markoff chain. Consider three nonvoid sets of states satisfying $A \leqq B \leqq C$, where A consists of a single state $\{a\}$ and $C$ of a single state $\{c\}$. Define

$$
Q(n ; a, c)=\operatorname{Pr}\{X(v) \in B \text { for } 1 \leqq v \leqq n-1, X(n)=c \mid X(0)=a\} \quad(n \geqq 1)
$$

and

$$
Q(0, a, c)= \begin{cases}0, & a \neq c \\ 1, & a=c\end{cases}
$$

If $L(n, m)=Q(n+m ; a, c)$ for $n, m \geqq 0$, then $L(n, m)$ is $\mathbf{R}_{r}$.

Proof. (i) Enumerating all the contingencies for the $m$-step transition probabilities, we obtain the relationship

$$
L(n, m)=\sum_{l} K(n, l) \tilde{K}(l, m),
$$

where $K(n, l)=\operatorname{Pr}\{X(n)=l \mid X(0)=0\}$ and $\tilde{K}(l, n)=\operatorname{Pr}\{X(m)=0 \mid X(0)=l\}$. Note that (2.22) depends on the fact that $\mathscr{P}$ is temporally homogeneous. It should be emphasized that (2.22) is also valid when $n=0$ and/or $m=0$.

By Theorem 2.6, $K(n, l)$ is $\operatorname{TP}_{r}$ for $n \geqq 0$ and $0 \leqq l<\infty$, and $\tilde{K}(l, m)$ is also $\mathrm{TP}_{r}$ for $m \geqq 0$ and $0 \leqq l<\infty$. Applying (0.4), we obtain

$$
L\left(\begin{array}{l}
n_{1}, n_{2}, \cdots, n_{v} \\
m_{1}, m_{2}, \cdots, m_{v}
\end{array}\right)=\sum_{0 \leqq l_{1}<l_{2}<\cdots<l_{v}} K\left(\begin{array}{l}
n_{1}, n_{2}, \cdots, n_{v} \\
l_{1}, l_{2}, \cdots, l_{v}
\end{array}\right) \tilde{K}\left(\begin{array}{c}
l_{1}, l_{2}, \cdots, l_{v} \\
m_{1}, m_{2}, \cdots, m_{v}
\end{array}\right)
$$

and it follows immediately that $L(n, m)$ is $\mathrm{TP}_{r}$, since each factor in the summand is non-negative for all $v \leqq r$. 
(ii) The proof of (ii) is similar to that of (i). We use Corollary 2.1.

(iii) Elementary probabilistic considerations, combined with the fact that $\mathscr{P}$ is temporally homogeneous, yield for $n, m \geqq 0$ :

$$
\begin{aligned}
L(n, m)= & \operatorname{Pr}\{X(v) \in B \text { for } 1 \leqq v \leqq n+m-1, X(n+m)=c \mid X(0)=a\} \\
= & \sum_{l \in B} \operatorname{Pr}\{X(v) \in B \text { for } 1 \leqq v \leqq n-1, X(n)=l \mid X(0)=a\} \\
& \quad \times \operatorname{Pr}\{X(v) \in B \text { for } 1 \leqq v \leqq m-1, X(m)=c \mid X(0)=l\} \\
= & \sum_{l \in B} K_{a}(n, l) \tilde{K}_{c}(l, m),
\end{aligned}
$$

where $K_{a}$ and $\tilde{K}_{c}$ are defined in the obvious way. By Theorem 2.2, we have that $K_{a}(n, l)$ is $\mathrm{TP}_{r}$ for $n \geqq 0$ and $a \leqq l<\infty$, which includes the set $B$. Theorem 2.1 implies that $\widetilde{K}_{c}(l, n)$ is $\mathrm{RR}_{r}$ for $m \geqq 0$ and $-\infty<l \leqq c$, again including the set $B$. Employing the method of (2.23) as in (i) leads to the desired conclusion.

Additional probability functions of $\mathrm{TP}_{r}$ Markoff matrix chains that are sign regular are given in $\S 10$ in the course of investigations concerning unimodality and limit theorems.

3. Total positivity for discrete-time, continuous-state Markoff processes. In this section $\mathscr{P}$ represents a Markoff process on the real line with transitions occurring at discrete units of time. A Borel field of subsets of the real line is given; a transition probability function is prescribed for each time period satisfying the standard measurability requirements; an appropriate fixed initial distribution is also specified.

In the matter of measurability we shall not burden the reader with unimportant details. Henceforth all sets and functions encountered are postulated to be measurable and well defined. The demonstration of these properties is usually fairly routine but sometimes tedious.

Let $X(v)(v=0,1,2, \cdots)$ denote the sequence of random variables which describe the process $\mathscr{P}$. The $n$th step transition probability function is assumed to be generated by a density $k_{n}(u, v)$ such that for any measurable set $E$,

$$
\operatorname{Pr}\{X(n) \in E \mid X(n-1)=u\}=K_{n}(u ; E)=\int_{E} k_{n}(u, v) d \mu(v) \quad(n \geqq 1),
$$

where $d \mu$ is a suitable sigma-finite measure. (Although we have postulated the existence of densities, the theory goes through in more general context. Thus, when densities do not exist we could phrase our conclusions directly in terms of the corresponding determinants involving $K_{n}(u, E)$ in the manner of $(0.5)$.)

The definition and notation of the preceding section carry over with the obvious adjustments. We now present the appropriate analogs of Theorems $2.1-2.7$. The proofs follow closely those of the preceding section and will therefore be omitted.

THEOREM 3.1. Let $\mathscr{P}$ be a $\mathrm{TP}_{r}$ Markoff process (not necessarily temporally homogeneous), and suppose $B$ and $C$ are nonvoid sets such that $B \leqq C$. Then 


$$
Q(n, x, C)=\operatorname{Pr}\{X(v) \in B \text { for } 1 \leqq v \leqq n-1, X(n) \in C \mid X(0)=x\}
$$

is $\mathrm{RR}_{r}$ for $-\infty<x<\infty$ and $n \geqq 1$.

In order to generalize part (iii) of Theorem 2.1 to the present situation, we introduce the following notation:

$$
\begin{gathered}
q(n ; x, y)=\iint_{\xi_{n-1} \in B \ldots \xi_{1} \in B} k_{1}\left(x_{1}, \xi_{1}\right) k_{2}\left(\xi_{1}, \xi_{2}\right) \cdots k_{n}\left(\xi_{n-1}, y\right) \\
\cdot d \mu\left(\xi_{1}\right) d \mu\left(\xi_{2}\right) \cdots d \mu\left(\xi_{n-1}\right) .
\end{gathered}
$$

Thus, the symbol $q$ will generically designate a density counter part to the function $Q$ of (3.2). Note the formula

$$
Q(n ; x, C)=\int_{C} q(n ; x, c) d \mu(c),
$$

which lends further justification to our notation.

THEOREM 3.2. Let $\mathscr{P}$ be a $\mathrm{TP}_{r}$ Markoff process, and suppose $B \leqq\{c\}$. Then $q(n ; x, c)$, defined as in (3.3), is $\mathrm{R}_{\mathrm{r}}$ for $-\infty<x \leqq c$ and $n \geqq 0$. (Here $q(0 ; x, y)=\delta(x-y)$, where $\delta$ is the Kronecker delta function, i.e.,

$$
\delta(x-y)= \begin{cases}0, & x \neq y, \\ \infty, & x=y,\end{cases}
$$

and $\int \delta(x-y) d \mu(y)=1$.) If $B \geqq\{c\}$, then $q(n ; x, c)$ is $\mathrm{TP}_{r}$ for $c \leqq x<\infty$ and $n \geqq 0$.

THEOREM 3.3. Let $\mathscr{P}$ be a temporally homogeneous $\mathrm{TP}_{r}$ Markoff process, and suppose $\{a\} \leqq B$. Then $q(n ; a, y)$, defined as in (3.3), is $\mathrm{TP}_{r}$ for $-\infty<y<\infty$ and $n \geqq 1$. If $q_{B}(0 ; a, y)$ denotes the delta function $\delta(a-y)$, then $q_{B}(n ; a, y)$ is $\mathrm{TP}_{r}$ for $a \leqq y<\infty$ and $n \geqq 0$.

The analog of Theorem 2.3 is

THEOREM 3.4. Let $\mathscr{P}$ be a spatially homogeneous TP $_{r}$ Markoff process, (in this case we require that $d \mu$ of (3.1) coincide with ordinary Lebesgue measure on the line) and let $B$ and $C$ denote nonvoid sets such that $B \leqq C$. Then

(3.5) $Q(n ; y, C)=\operatorname{Pr}\{X(v) \in B+y$ for $1 \leqq v \leqq n-1, X(n) \in C+y \mid X(0)=a\}$

is $\mathrm{TP}_{r}$ for $-\infty<y<\infty$ and $n \geqq 1$. If $C$ contracts to a single point $\{c\}$, we interpret. (3.5) as the density function $q_{B}(n ; y, c)$ defined analogously to (3.3). Then $q(n ; y, c)$ is $\mathrm{TP}_{r}$ for $-\infty<y<\infty$ and $n \geqq 1$. Under the usual definition for $n=0, q(n ; y, c)$ is $\operatorname{TP}_{r}$ for $n \geqq 0$ and $\infty>y \geqq a-c$.

THEOREM 3.5. Let $\mathscr{P}$ be a temporally homogeneous $\mathrm{TP}_{r}$ Markoff process, and suppose $\{a\} \leqq B \leqq C$. Define 


$$
\Lambda_{n}=\operatorname{Pr}\{X(v) \in B \text { for } 1 \leqq v \leqq n-1, X(n) \in C \mid X(0)=a\}
$$

then

$$
L(n, m)=\Lambda_{n+m} \text { is } \mathrm{R}_{r} \text { for } n, m \geqq 1 .
$$

We close this section by stating the analogs of Theorems 2.4-2.7. In all of these we assume that the Markoff process $\mathscr{P}$ is $\mathrm{TP}_{r}$. Additional assumptions are stipulated as required.

THEOREM 3.6. Suppose $\mathscr{P}$ repiesents a stochastic motion with non-negative drift, and let $k^{(n)}(x, c)$ denote the $n$-step transition density from initial position $x$ to final position $c$. Then $k^{(n)}(x, c)$ is $\mathrm{R}_{r}$ for $-\infty<x \leqq c$ and $n \geqq 1$.

THEOREM 3.7. Suppose $\mathscr{P}$ is spatially homogeneous and has non-negative drift. Let $k^{(n)}(y)=k_{1}(y) * \cdots * k_{n}(y)$, where $*$ designates convolution in the sense $k_{1}(y) * k_{2}(y)=\int k_{1}(y-\xi) k_{2}(\xi) d \xi$. Then

(i) $k^{(n)}(a, y)$ is $\operatorname{TP}_{r}$ for $n \geqq 1$ and $y \geqq a$.

(ii) $L(m, n)=k^{(m+n)}(x, y)$ is $\mathrm{R}_{r}$ for $m, n \geqq 1$.

THEOREM 3.8. Suppose $\mathscr{P}$ is temporally homogeneous and has only the non-negative real line as its state space. Then

(i) $k^{(n)}(0, y)$ is $\mathrm{TP}_{r}$ for $0 \leqq y<\infty$ and $n \geqq 1$.

(ii) $k^{(n)}(y, 0)$ is $\operatorname{TP}_{r}$ for $0 \leqq y<\infty$ and $n \geqq 1$.

(iii) $Q(n, y)=\int_{y}^{\infty} k^{(n)}(0, \xi) d \mu(\xi)$ is $\operatorname{TP}_{r}$ for $0<y<\infty$ and $n \geqq 1$.

(iv) $k^{(n+m)}(0,0)$ is $\mathrm{TP}_{r}$ for $n, m \geqq 1$.

THEOREM 3.9. Suppose $\mathscr{P}$ is temporally homogeneous and has non-negative drift. Then

(i) $k^{(n)}(a, y)$ is $\mathrm{TP}_{r}$ for $a \leqq y<\infty$ and $n \geqq 1$,

(ii) $L(m, n)=k^{(m+n)}(x, y)$ is $\mathrm{R}_{r}$ for $m, n \geqq 1$,

(iii) $L(m, n)=\operatorname{Pr}\{X(v)<y$ for $1 \leqq v \leqq n+m-1, X(n+m) \geqq y \mid X(0)=x\}$ is $\mathrm{R}_{r}$ for $m, n \geqq 1$.

In $\S 8$ we give several examples and applications of these results.

4. Total positivity for continuous-time, discrete-state Markoff processes. In this section we develop the analogs of Theorems 2.1-2.7 for the case of a continuoustime, temporally homogeneous Markoff chain. The state space coincides with the integers of the real line (or an interval subset of it), and the time variable $t$ traverses the positive axis. The process is assumed to be measurable, separable and all finite states are taken to be stable. Without loss of generality we postulate that the path functions are normalized to be right continuous. We let $X(t)$ represent the process and

$$
P(t ; i, j)=\operatorname{Pr}\{X(t)=j \mid X(0)=i\}
$$

its transition probability function. 
The fundamental hypothesis underlying the results of the preceding sections is that the one-step transition probability function is totally positive in the initialand final-state variables. In dealing with Markoff processes in continuous time, we must strengthen this hypothesis and require that $P(t ; i, j)$ be $\mathrm{TP}_{r}$ in the variables $i$ and $j$ for each $t>0$. Under this condition (actually $\mathrm{TP}_{2}$ is sufficient)( $\left.{ }^{8}\right)$ the process is identifiable as an extended birth-and-death process including as special cases the pure birth processes and the pure death processes [22]. (The usual terminology of birth-and-death processes refers to the situation where the nonnegative integers are the state space. We employ the term "extended birth-anddeath process" to emphasize the fact that the state space may run over all the integers.)

Birth-and-death processes have the property that almost all realizations are continuous paths in the sense that the only changes of state are to neighboring states. For a thorough discussion of birth-and-death processes we refer the reader to [16], [17], and [22]. We borrow the following facts from these sources. The density $P(t ; i, j)$ is continuous as a function of $t$. All first-passage distributions associated with the states of the process have continuous densities.

One approach to the analysis of continuous-time processes is to approximate by suitable discrete-time processes; we follow this viewpoint in extending the results of $\$ 2$. Let $h$ be a small positive number. By considering the realization of the process only at the specific times $t_{n}=n h(n=1,2, \cdots)$, we induce a temporally homogeneous discrete-time Markoff chain, $\mathscr{P}_{h}$, whose one-step transition probability matrix is $P(i, j)=P(h ; i, j)$. In terms of the path functions $X(t)$, the derived process is represented by $X(n h)$.

By assumption $P(h ; i, j)$ is $\mathrm{TP}_{\infty}$ (see footnote 8$)$ and the associated transition probability functions, absorption probability functions and recurrence probabilities as determined in $\S 2[(2.1),(2.16)$, and (2.24)] are appropriately sign regular. For example, let $B=\left(-\infty, j_{0}\right]$ and $C=\left[j_{0}+1, \infty\right)$, and define

$$
Q(n h, i, C)=\operatorname{Pr}\{X(v h) \in B \text { for } 1 \leqq v \leqq n-1, X(n h) \in C \mid X(0)=i\},
$$

with $i$ in $B$. We conclude by Theorem 2.1 that $Q_{B}(n h, i, C)$ is $\mathrm{R}_{\infty}$ for $n \geqq 1$ and $-\infty<i \leqq j_{0}$. Let $T_{i}(h, C)$, apart from the multiplying constant $h$, denote the random variable equal to the time of first entrance into $C$ for the process $X(n h)$ and initial state $i$.

Consider for the $X(t)$ process the first-passage distribution function into the set $C$ with initial state $i\left(-\infty<i<j_{0}+1\right)$. Let $T_{i}(C)$ denote the random variable equal to the infimum of $t$ values for which $X(t)$ is in $C$ under the initial condition $X(0)=i$; in other words, $T_{i}(C)$ is the first-passage time into the set of states $C$ with $X(0)=i$. Since the path functions are "continuous" (i.e., only jumps of

(8) It is proved in [22] that a continuous-time stable Markoff chain is $\mathbf{T P}_{2}$ if and only if it is $\mathbf{T P} \mathbf{P}_{\infty}$. 
magnitude 1 occur), first passage necessarily occurs through the state $j_{0}+1$. Thus $T_{i}(C)$ can equivalently be defined as the time of first passage from state $i$ into state $j_{0}+1$. It is easily seen from the discussion of [17] that $T_{i}(C)$ has a continuous density function with respect to Lebesgue measure; we denote this function by $q\left(t ; i, j_{0}\right)$. Formally,

$$
\int_{t}^{\infty} q\left(\xi ; i, j_{0}\right) d \xi=\operatorname{Pr}\left\{X(\tau) \in B \text { for } 0 \leqq \tau<t, T_{i}(C) \geqq t \mid X(0)=i\right\} .
$$

Now in (4.1) let $h \rightarrow 0$. Since the paths are "continuous" and bonafide right continuous we infer that the random variable $T_{i}(h, C)$ converges almost surely to $T(C)$. Hence their distributions converge and

$$
\phi_{i}(t, h, C)=\sum_{n ; t \leqq n h<t+u} Q(n h, i, C) \rightarrow \int_{t}^{t+u} q\left(\xi, i, j_{0}\right) d \xi=R\left(t ; u, i, j_{0}\right)=R, h \rightarrow 0+,
$$

for arbitrary fixed $t>0, u>0$. Since $\phi_{i}(t, h, C)$ is $\mathrm{RR}_{\infty}$ for $t>0$ and $-\infty<i \leqq j_{0}$ keeping the other variables fixed the same property persists for the limiting function. Dividing $R$ by $u$ and letting $u$ approach zero, since $q$ is continuous we conclude that $q\left(t ; i, j_{0}\right)$ is $\mathrm{RR}_{\infty}$ for $t>0$ and $i,-\infty<i \leqq j_{0}$.

The preceding discussion is now summarized in the statement of the following theorem.

THEOREM 4.1. Let $\mathscr{P}$ denote a continuous-time, temporally homogeneous Markoff chain whose transition probability function $P(t ; i, j)$ is $\mathrm{TP}_{2}$ for every $t>0$. Let the random variable $T_{i j}$ represent the time of first passage from state $i$ to state $j$, and let $q(t ; i, j)$ denote its density function (see the discussion preceding the theorem). Then

(i) When $i<j$, the function $q(t ; i, j)$ is $\mathrm{R}_{\infty}$ in the variables $t>0$ and $i$ $(-\infty<i<j)$.

(ii) When $i>j$, the function $q(t ; i, j)$ is $\mathrm{TP}_{\infty}$ in the variables $t>0$ and $i(j<i<\infty)$.

(iii) If in addition to the hypothesis we also require that $\mathscr{P}$ is spatially homogeneous, then $q(t ; i, j)$ as given in (i) is $\mathrm{TP}_{\infty}$ in the variables $t>0$ and $j(j>i)$.

There exist only two prime examples of spatially homogeneous birth-and-death processes: the classical Poisson process, and the standard coin-tossing process (the coin could be biased) featuring an independent exponential waiting time between successive tosses.

We now list, without attempting to be exhaustive, further results analogous to those of $\S 2$. In each case the result is demonstrated by appealing to the corresponding theorem of $\$ 2$ for a discrete-time process which approximates the desired probability function (see the argument preceding Theorem 4.1). Henceforth, unless stated explicitly to the contrary we assume that the hypotheses of Theorem 
4.1 apply. The notation and terminology are the obvious adaptations of those of $\S 2$. The proofs are omitted.

THEOREM 4.2. Let $\mathscr{P}$ be a $\mathrm{TP}_{2}$ process with non-negative drift, i.e., a pure birth process not necessarily spatially homogeneous. Then

(i) $P(t ; i, j)$ is $\mathrm{RR}_{\infty}$ in the variables $t>0$ and $i(-\infty<i \leqq j)$ and $\mathrm{TP}_{\infty}$ in the variables $t>0$ and $j \geqq i$.

(ii) $q(t ; i, j)$ is $\mathrm{TP}_{\infty}$ in the variables $t>0$ and $j(j>i)$.

(iii) $L(t, s)=P(t+s ; i, j)$ is $\mathrm{RR}_{\infty}$ for $t, s>0$.

(iv) $K(t, s)=q(t+s ; i, j)$ is $\mathrm{RR}_{\infty}$ for $t, s>0$.

The proof of (ii) does not quite follow by the methods outlined above since we have not proved the discrete version in general form. However, in the present context we can give a proof of (ii) based on Theorem 3.7. Indeed, since the process is a pure birth process it follows that $q(t ; i, j)$ is the density function of a sum of $j-i$ independent exponentially distributed random variables

$$
T_{i j}=T_{i, i+1}+T_{i+1, i+2}+\cdots+T_{j-1, j}
$$

where $T_{k, k+1}$ denotes the first-passage time from state $k$ to state $k+1$. The result now follows by appealing to Theorem 3.7, part (i), with $a=0$.

The proofs of the other parts proceed as suggested above.

THEOREM 4.3. Suppose the process $\mathscr{P}$ satisfies the hypothesis of Theorem 4.1 and admits only the non-negative integers as its state space. Then

(i) $P(t ; 0, j)$ is $\mathrm{TP}_{\infty}$ for $t>0$ and $j \geqq 0$.

(ii) $P(t, j, 0)$ is $\mathrm{TP}_{\infty}$ for $t>0$ and $j \geqq 0$.

(iii) $\sum_{k=j}^{\infty} P(t ; 0, k)$ is $\mathrm{TP}_{\infty}$ for $t>0$ and $j \geqq 0$.

(iv) $P(t+s, 0,0)$ is $\mathrm{TP}_{\infty}$ for $t, s>0$.

We remark in closing that most of these theorems extend to the case of continuous-time Markoff chains which are not necessarily temporally homogeneous.

5. Total positivity for continuous-time, continuous-state Markoff processes. The elements of the preceding theory extend to the continuous-time, continuousstate process with minor changes and the obvious modifications in notation, terminology, definitions, and proofs. We shall state the principal analogs of the results of $\S 1$ without proof.

Let $\mathscr{P}$ represent a temporally homogeneous separable strong Markoff process with the real line as its state space and with a continuous-time parameter. Furthermore, assume that the process has a continuous transition density function $p(t ; x, y)$ such that the transition probability function is calculated according to the formula

$$
P(t ; x, E)=\int_{E} p(t ; x, y) d \mu(y),
$$


where $\mu$ is $\sigma$-finite regular measure. The process can be described by the set of random variables $X(t)$ and

$$
P(t ; x, E)=\operatorname{Pr}\{X(t) \in E \mid X(0)=x\} .
$$

Our basic assumption asserts that $p(t ; x, y)$ for each $t>0$ is $\mathrm{TP}_{\infty}$ with respect to the real variables $x$ and $y$. Total positivity for all $t>0$ means essentially that the path functions of the process are continuous. More precisely, subject to very mild conditions, it can be shown (see [20]) that the following three properties are equivalent:

(a) $p(t ; x, y)$ is $\mathrm{TP}_{2}$ in the variables $x$ and $y$ for each $t>0$.

(b) $p(t ; x, y)$ is $\mathrm{TP}_{\infty}$ in the variables $x$ and $y$ for each $t>0$.

(c) The process $\mathscr{P}$ has a realization with almost every path function continuous.

Continuity of path function is to be understood in the extended sense of including the boundaries of the state space. Hereafter we avoid pathology and uninteresting technical difficulties by postulating that the process $\mathscr{P}$ indeed possesses properties (a)-(c). We refer to a forthcoming monograph of Itô and McKean where the continuity properties of linear diffusion process are well established.

In order to state our main results we introduce the relevant probability functions associated with first-passage time into specified sets. Explicitly, let $T(x, y)$ denote the random variable equal to the time the particle first reaches $y$, having started initially at $x$. Since the path functions are almost all continuous, $T(x, y)$ is well defined. Let $U$ be a Borel set on the positive real line, and define

$$
Q(U ; x, y)=\operatorname{Pr}\{T(x, y) \in U\} .
$$

In order to avoid inessential complications we frequently postulate the existence of a density function $q(t ; x, y)$ such that

$$
Q(U ; x, y)=\int_{U} q(t ; x, y) d t .
$$

This assumption holds in the vast majority of important examples and under extremely mild conditions [28]. We should emphasize that $q$ is not necessarily a density function in the strict sense: i.e., the integral in (5.4) extended over the full line may be less than 1 . This is immaterial for our purposes.

With the above terminology and definitions established, we now state the main theorems of this section.

THEOREM 5.1. Let $\mathscr{P}$ be a temporally homogeneous strong Markoff process with a continuous transition density function (5.1) which is $\mathrm{TP}_{\infty}$. Then

(i) The first-passage function $q(t ; x, y)$ is $\mathrm{R}_{\infty}$ in the variables $t>0$ and $x<y$. Also, $q(t ; x, y)$ is $\mathrm{TP}_{\infty}$ in the variables $t>0$ and $x>y$.

(ii) If $\mathscr{P}$ is spatially homogeneous, i.e., if $P(t, x, E)=P(t, 0, E-x)$, then $q(t ; x, y)=q(t ; y-x)$ is $\mathrm{TP}_{\infty}$ in the variables $t>0$ and $y>x$. 
By imposing further conditions on the process, we may infer more special results corresponding to Theorems 3.6-3.9.

THEOREM 5.2. Let the assumptions of Theorem 5.1 apply, and suppose in addition that the state space of $\mathscr{P}$ is the non-negative real line. Then $p(t ; 0, y)$ is $\mathrm{TP}_{\infty}$ for $t>0$ and $y \geqq 0$. Similarly, $p(t ; y, 0)$ is $\mathrm{TP}_{\infty}$ for $t>0$ and $y \geqq 0$. Also, $p(t+s ; 0,0)$ is $\mathrm{TP}_{\infty}$ for $t, s>0$.

TheOREM 5.3. Suppose $\mathscr{P}$ satisfies the conditions of Theorem 5.1 and has non-negative drift. Then

(i) $p(t ; x, y)$ is $\mathrm{RR}_{\infty}$ for $t>0$ and $x \leqq y$, and $\mathrm{TP}_{\infty}$ for $t>0$ and $y \geqq x$.

(ii) $q(t ; x, y)$ is $\mathrm{TP}_{\infty}$ for $t>0$ and $y>x$.

(iii) $L(t, s)=p(t+s ; x, y)$ is $\mathrm{R}_{\infty}$ for $t, s>0$.

(iv) $K(t, s)=q(t+s ; x, y)$ is $\mathrm{RR}_{\infty}$ for $t, s>0$.

Theorems 5.1-5.3 are proved by the same methods that gave the theorems of $\S 4$ as limiting cases of those of $\$ 2$. We discretize the time scale $\left(t_{n}=n h\right.$, with $h>0$ ) and thus induce a discrete-time process of the kind studied in $\S 3$. For this process we form the transition probability density $p(n h ; x, y)$, and the first-passage probability density $q(n h ; x, y)$ from initial position $x$ into the set $(y, \infty)$. Letting $h \rightarrow 0+$ leads in the limit to the probability functions $q(t ; x, y)$ and $p(t ; x, y)$ of Theorems 5.1 and 5.2. To be rigorous it is necessary to operate in terms of the cumulative distributions in the manner of $\$ 4$. Once the cumulants are proved to be sign regular then differentiation yields that the corresponding densities also are sign regular of the same type. Continuity of the path functions of the process is essential to justify the limiting procedure. The formal arguments being straightforward elaborations, we omit the details (cf. §4).

6. Variation-diminishing properties. As noted in $\$ 1$, sign-regular functions $K(x, y)$ of order $r$ defined on $X \otimes Y$ where $X$ and $Y$ are linear sets, generate variation-diminishing transformations in the following manner.

I. Let $f(y)$ be piecewise continuous bounded and such that $V(f) \leqq r-1$, where $V(f)$ denotes the number of sign changes of $f$. Suppose that $g(x)$ is also piecewise continuous and is computed from $f$ by the linear transformation

$$
g(x)=\int K(x, y) f(y) d \mu(y)
$$

where $d \mu(y)$ is a non-negative sigma-finite measure and the integral converges absolutely. Then $V(g) \leqq(f)$, provided $K$ is sign regular of order $r$.

II. Totally positive kernels are essentially characterized by Property I and the further property that if $V(g)=V(f)$, then $g(x)$ and $f(x)$ exhibit the same arrangement of sign changes when the argument $x$ traverses its domain from left to right.

The results of $\$ \S 2-5$ furnish many functions which generate sign-variation- 
diminishing transformations. For example, under the conditions of Theorem 2.6, $V(\beta) \leqq V(\alpha)$ for any pair of sequences $\beta=\left(\beta_{n}\right)$ and $\alpha=\left(\alpha_{j}\right)$ connected by the transformation

$$
\beta_{n}=\sum_{j=0}^{\infty} P_{0 j}^{n} \alpha_{j} \quad(n=0,1, \cdots),
$$

where $\alpha_{j}$ is uniformly bounded and $V(\alpha)<r$. As a collorary of this property, plus the condition that

$$
\sum_{j=0}^{\infty} P_{0 j}^{n}=1
$$

for all $n$, we deduce that whenever $\alpha_{j}$ is decreasing, $\beta_{n}$ is also decreasing. Indeed, the sequence $\left\{\alpha_{j}-c\right\}$ for any real constant $c$ possesses at most one sign change from + to - values as $j$ traverses the set of non-negative integers. But $\left\{\alpha_{j}-c\right\}$ is transformed under (6.2) into the sequence $\left\{\beta_{n}-c\right\}$, which therefore also admits at most one sign change. If it actually changes sign, the change must be from + to - values for $n$ increasing. Since this inference holds for every real constant $c$, it follows that $\beta_{n}$ is decreasing.

The fact that $P_{0 j}^{n}$ of Theorem 2.6 is $\mathrm{TP}_{r}$ implies also that the transformation

$$
\mu_{j}=\sum_{k=1}^{\infty} \lambda_{k} P_{0 j}^{n_{k}} \quad\left(j=0,1,2, \cdots ; n_{1}<n_{2}<n_{\mathfrak{3}}<\cdots\right)
$$

is variation-diminishing when applied to any sequence $\left(\lambda_{k}\right)$ for which $V(\lambda) \leqq r-1$ provided (6.3) converges absolutely for every $j$. In contrast, the kernel $Q(n, j)=P_{i j}^{n}$, with $i \neq 0$, is not sign regular (see the paragraph on counterexamples of §2) and therefore the transformation

$$
\sum_{k=1}^{\infty} c_{k} Q\left(n_{k}, j\right)=d_{j} \quad(j=0,1, \cdots)
$$

is not necessarily sign-variation-diminishing. Nevertheless, in Theorem 6.1 below we are able to provide an estimate of the number of sign changes in the sequence $d_{j}$ of (6.4). In order to formulate the result in its most appropriate setting, we revert to the situation where the state space is the set of all integers, positive and negative.

THEOREM 6.1. Let $\mathscr{P}$ denote a temporally homogeneous Markoff chain whose state space is the set of all integers, and let $\mathscr{P}$ be $\mathrm{TP}_{2 r}$. Then, given nonzero real numbers $c_{1}, \cdots, c_{v}$ (with $v \leqq r$ ) and an increasing sequence of non-negative integers $n_{k}$, the transformed sequence

$$
\sum_{k=1}^{v} c_{k} P_{i j}^{n_{k}}=d_{j} \quad(-\infty<j<\infty)
$$

can change signs at most $2 v-2$ times. Here $i$ is held fixed. 
Proof. The proof proceeds by induction on $v$. Observe first that the theorem is trivially valid for $v=1$. We assume that the theorem holds for $v-1 \geqq 0$ and prove that it holds for $v$. We may write

$$
d_{j}=\sum_{k=1}^{v} c_{k} \sum_{l=-\infty}^{\infty} P_{i l}^{n_{k}-n_{1}} P_{l j}^{n_{1}}=\sum_{l=-\infty}^{\infty} P_{l j}^{n_{1}}\left(c_{1} \delta_{i l}+\sum_{k=2}^{v} c_{k} P_{i l}^{n_{k}-n_{1}}\right)
$$

where $\delta$ represents as usual the delta function. By the induction hypothesis the sequence

$$
\gamma_{l}=\sum_{k=2}^{v} c_{k} P_{i l}^{n_{k}-n_{1}}
$$

has at most $2 v-4$ sign changes; hence $c_{i} \delta_{i l}+\gamma_{l}(-\infty<l<\infty)$ has at most $2 v-2$ sign changes since $\delta_{i l}$ has only one nonzero component. Since $P_{l j}^{n_{1}}$ is $\mathrm{PT}_{2 r}$ for fixed $n_{1}$, it is variation-diminishing, and the desired conclusion follows by using this property in (6.6).

In Theorem 6.3 below we shall refer to relation (6.6) and use the stronger property enunciated for TP transformations as stated in Property II at the beginning of this section.

The same result can be achieved when we regard the transformation (6.5) as a sequence in the variable $i$ (the initial state) with $j$ held fixed.

THEOREM 6.2. Let $\mathscr{P}$ be a $\mathrm{TP}_{2 r}$ Markoff chain (not necessarily temporally homogeneous), let $n_{k}$ be an increasing sequence of non-negative integers, and let $c_{i}(i=1, \cdots, v)$ be nonzero real numbers. Then

$$
d_{i}=\sum_{k=1}^{r} c_{k} P_{i j}^{n_{k}} \quad(-\infty<i<\infty)
$$

can change signs at most $2 v-2$ times. Here $j$ is fixed.

The proof follows that of Theorem 6.1 except in one respect. The decomposition of $\operatorname{Pr}\left\{X\left(n_{k}\right)=j \mid X(0)=i\right\}$ in (6.6) is now done in terms of the initial $n_{1}$ transitions rather than the last $n_{1}$ transitions, and takes the form

$$
P_{i j}^{n_{k}}=\sum_{l=-\infty}^{\infty} P_{i l}^{n_{1}} Q_{l j}^{n_{k}, n_{1}}
$$

where we write $Q_{l j}^{n_{k}, n_{1}}$ for $\operatorname{Pr}\left\{X\left(n_{k}\right)=j \mid X\left(n_{1}\right)=l\right\}$ to emphasize the fact that the process is not necessarily temporally homogeneous.

The statement of Theorem 6.1 can be considerably sharpened when $\mathscr{P}$ is symmetric (i.e., if $\mathrm{P}_{i, j}=P_{-i,-j}$ ), as follows.

THEOREM 6.3. Suppose $\mathscr{P}$ is symmetric and satisfies the hypothesis of Theorem 6.1. Then for $v \leqq r$,

$$
V\left(\sum_{k=1}^{\nu} c_{k} P_{0 j}^{n_{k}}\right) \leqq 2 V\left(c_{k}\right)
$$


If equality holds, then

$$
\operatorname{sign} c_{1}=\left\{\begin{array}{c}
\text { sign of the first nonzero term of the sequence } \\
\left\{\sum_{k=1}^{v} c_{k} P_{0 j}^{n_{k}}\right\}_{i=0}^{\infty}
\end{array}\right.
$$

and

$$
\operatorname{sign} c_{v}=\lim _{l \rightarrow \infty} \operatorname{sign}\left(\sum_{k=1}^{v} c_{k} P_{0 l}^{n_{k}}\right)
$$

Moreover

$$
Q(n, j)=P_{0 j}^{n} \text { is } \mathrm{TP}_{r} \text { for } n \geqq 0 \text { and } j \geqq 0 \text {. }
$$

REMARK. The number of sign changes of the sequence

$$
\sum_{k=1}^{v} c_{k} P_{0 j}^{n_{k}}
$$

is counted as $j$ traverses the full set of integers. On the other hand, the total positivity property announced in (6.9) is necessarily restricted to the domain indicated.

Proof. The proof of (6.8) proceeds by induction on $v$. The case of $v=1$ is trivial; we next assume that (6.8) holds for $v-1$ and seek to prove that it holds for $v$. To this end, we distinguish two cases.

Case (i) (see first (6.6)).

$$
V\left(\sum_{k=2}^{v} c_{k} P_{0 l}^{n_{k}-n_{1}}\right)=2 V\left(c_{2}, c_{3}, \cdots, c_{v}\right)
$$

By the induction hypothesis the sign of

$$
z_{l}=\sum_{k=2}^{v} c_{k} P_{0 l}^{n_{k}-n_{1}}
$$

is that of $c_{2}$ for $l=0$ and that of $c_{v}$ for $l$ sufficiently large. Clearly $c_{1} \delta_{0 l}+z_{l}$ has either no new sign changes or at most two new sign changes, according as $c_{1}$ and $c_{2}$ are of the same sign or not. Since $P_{l j}^{n_{1}}$ is $\mathrm{TP}_{2 r}$, we infer that in either case

$$
V\left(\sum_{k=1}^{v} c_{k} P_{0 l}^{n_{k}}\right) \leqq 2 V\left(c_{1}, c_{2}, \cdots, c_{v}\right)
$$

If equality holds in (6.10) then by virtue of Property II stated at the start of this section and (6.6) we deduce that the sequences

$$
x_{l}=\sum_{k=1}^{v} c_{k} P_{0 l}^{n_{k}} \text { and } y_{l}=c_{1} \delta_{0 l}+\sum_{k=2}^{v} c_{k} P_{0 l}^{n_{k}-n_{1}}
$$


exhibit the same arrangement of sign changes. But we know from the induction hypothesis that the sign of the second sequence for large $l$ is that of $c_{v}$, and thus the same is true for the first sequence. Moreover, since

$$
\sum_{k=1}^{v} c_{k} P_{0 l}^{n_{k}}=x_{l}
$$

is symmetric in $l$ (i.e., $x_{l}=x_{-1}$ ), we infer that the sequence $x_{0}, x_{1}, x_{2}, \cdots$ exhibits exactly $V\left(c_{1}, c_{2}, \cdots, c_{v}\right)$ sign changes. But the sign of $x_{l}$ for $l$ large is that of $c_{v}$; hence $x_{0}$ has the sign $c_{1}$, and the induction step is completed.

Case (ii).

$$
V\left(\sum_{k=2}^{v} c_{k} P_{G l}^{n_{k}-n_{1}}\right)<2 V\left(c_{2}, c_{3}, \cdots, c_{v}\right)
$$

(The difference is necessarily an even number by virtue of the symmetry hypothesis.)

By the same reasoning as in Case (i) we deduce (6.10). It remains to discuss the case of equality in (6.10). If equality holds, then $y_{l}$ as defined in (6.11) satisfies

$$
V\left(y_{l}\right)=2 V\left(c_{1}, c_{2}, \cdots, c_{v}\right) \text {. }
$$

Examination of the argument of (6.6) reveals that the assumption of Case (ii) and relation (6.12) are compatible if and only if $c_{1}$ and $c_{2}$ are of the same sign and $z_{0}=\sum_{k=2}^{v} c_{k} P_{00}^{n_{k}-n_{1}}$ has the sign opposite to that of $c_{1}$. A comparison of (6.12) and the assumption of Case (ii) implies

$$
V\left(\sum_{k=2}^{v} c_{k} P_{0 i}^{n_{k}-n_{1}}\right)=2\left[V\left(c_{2}, c_{3}, \cdots, c_{v}\right)-1\right]
$$

By symmetry the number of sign changes of

$$
\sum_{k=2}^{v} c_{k} P_{0 l}^{n_{k}-n_{1}}=z_{l}
$$

over the range $l \geqq 0$ is

$$
V\left(c_{2}, c_{3}, \cdots, c_{v}\right)-1 \text {. }
$$

Now suppose the sign of $z_{l}$ for $l$ sufficiently large is opposite to that of $c_{v}$. It follows from (6.13) that $\operatorname{sign} z_{0}=\operatorname{sign} c_{2}=\operatorname{sign} c_{1}$, in contradiction to what was proved before. Thus for $l$ sufficiently large $z_{l}$ and $y_{l}$ have the same sign as $c_{v}$. The argument of Case (i) now shows that

$$
\operatorname{sign}\left[\sum_{k=1}^{v} c_{k} P_{00}^{n_{k}}\right]=\operatorname{sign} c_{1},
$$

and the induction proof is completed.

By symmetry, 


$$
V_{+}\left(\sum_{k=1}^{v} c_{k} P_{0 l}^{n_{k}}\right) \leqq V\left(c_{k}\right)
$$

where $V_{+}$signifies that the variations in sign are counted only for $l \geqq 0$. Assertion (6.9) is now proven as follows. Let $Q\left(\begin{array}{l}j_{1}, \ldots, j_{v} \\ n_{1}, \ldots, n_{v}\end{array}\right)$ be any nonzero determinant formed from $Q(j, n)=P_{0 j}^{n}(n, j \geqq 0)$ and let $U$ be the matrix

$$
U=\left(\begin{array}{ccc}
P_{0 j_{1}}^{n_{1}} & \cdots & P_{0 j_{1}}^{n_{\nu}} \\
\vdots & & \\
P_{0 j_{v}}^{n_{1}} & \cdots & P_{0 j_{v}}^{n_{\nu}} \\
P_{0 j_{v}+1}^{n_{1}} & \cdots & P_{0 j_{v}+1}^{n_{\nu}}
\end{array}\right) .
$$

Since $U$ is of rank $v$ and $V(U c) \leqq V(c)\left(c=\left(c_{1}, \cdots, c_{v}\right)\right)$ it follows [15] that $U$ is $\mathrm{SR}_{v}$ (i.e., all subdeterminants of $U$ of fixed size $m$ have the same sign $\varepsilon_{m}$ ). Now let $U_{1}$ be the square matrix composed of the first $v$ rows of $U$ and let $\bar{c}$ be the solution of the system of equations

$$
U_{1} \bar{c}=\left(1,-1,1, \cdots,(-1)^{v+1}\right) .
$$

Then since $v-1 \leqq V\left(U_{1} \bar{c}\right) \leqq V(\bar{c}) \leqq v-1$, we conclude from the first part of the theorem that $\bar{c}_{1}>0$. Solving for $\bar{c}_{1}$ it follows, using the fact that $U$ is $\mathrm{SR}_{v}$, that sign $Q\left(\begin{array}{l}j_{1}, \ldots, j_{v} \\ n_{1}, \ldots, n_{v}\end{array}\right)=\varepsilon_{v-1}$. Proceeding by induction we then have that $\operatorname{sign} Q\left(\begin{array}{c}j_{1}, \ldots, j_{v} \\ n_{1}, \ldots, n_{v}\end{array}\right)=\varepsilon_{1}$, so that

$$
Q\left(\begin{array}{l}
j_{1}, \cdots, j_{v} \\
n_{1}, \cdots, n_{v}
\end{array}\right)>0
$$

The results of Theorems 6.1-6.3 go over directly to the case of discrete-time, continuous-state Markoff processes. We shall not burden the reader by stating all the possibilities. The following theorem is of some interest.

THEOREM 6.4. Let $\mathscr{P}$ be a temporally homogeneous discrete-time Markoff process with the one-step transition continuous density function $k(x, y)$ $(-\infty<x, y<\infty)$, and suppose $\mathscr{P}$ is $\operatorname{TP}_{2 r}$. Let $k^{(n)}(x, y)$ denote the $n$-step transition density function with initial state $x$ and final state $y$ as defined explicitly in (3.1). Then for $x$ fixed, $v \leqq r$, and $n_{l}$ an increasing set of positive integers,

$$
V\left(\sum_{l=1}^{v} c_{l} k^{n_{l}}(x, y)\right) \leqq 2 v-2,
$$

where $V$ denotes the number of variations in sign counted with respect to the variable $y(-\infty<y<\infty)$. If $\mathscr{P}$ is symmetric, then $k^{n}(0, y)=Q(n, y)$ is $\operatorname{TP}_{r}$ for $n \geqq 1$ and $y \geqq 0$, and 


$$
V_{+}\left(\sum_{l=1}^{\nu} c_{l} k^{n_{l}}(0, y)\right) \leqq V\left(c_{l}\right),
$$

where $V_{+}$refers to the number variations on the positive real line $y \geqq 0$.

The proof is an obvious adaptation of the Markoff chain case and will be omitted. Finally, the results for the symmetric case extend to the Markoff process in continuous time. The analysis proceeds by introducing the appropriate discretetime approximations to which Theorems 5.1 and 6.3 are applicable (see $\S \S 4$ and 5). The limit is then approached by making the time interval between transitions shrink to zero. The result is as follows:

THEOREM 6.5. Let $\mathscr{P}$ be a temporally homogeneous continuous-time symmetric Markoff process which is $\mathrm{TP}_{2 r}$. Suppose $p(t ; x, y)(-\infty<x, y<\infty)$ is continuous and represents the transition density in time $t$ with initial state $x$ and final state $y$. Then $p(t ; 0, y)$ is $\mathrm{TP}_{r}$ for $t>0$ and $y \geqq 0$.

\section{Composition of totally positive kernels.}

1. Starting with the sign-regular character of the transition density function we constructed other sign-regular probability functions involving the time variable and one of the state variables. We may now compound stochastic processes and in this way secure new sign-regular functions. This can be done by taking the time scale of the process itself random. Consider the following example. Let $f$ be a Pólya frequency density $\left(\mathrm{PF}_{\infty}\right)$ of a non-negative random variable which is continuous, and let $X_{i}$ denote random variables that are identically and independently distributed according to $f$. Let $\Lambda$ designate an interval of the real line and let $N(\lambda)$ for each $\lambda$ in $\Lambda$ be a random variable whose possible values are the nonnegative integers. Suppose $N(\lambda)$ has a density function $g(n, \lambda)$ which is $\mathrm{TP}_{r}$. Then the density function $h(x, \lambda)$ of $S(\lambda)=X_{1}+X_{2}+\cdots+X_{N(\lambda)}$ (for $N(\lambda)=0$ we put $S(\lambda)=0$ ) is $\mathrm{TP}_{r}$ in the variables $x>0$ and $\lambda$ in $\Lambda$. To verify this, we invoke the law of total probabilities, obtaining the explicit expression

$$
h(x, \lambda)=\sum_{n=0}^{\infty} f^{[n]}(x) g(n, \lambda)
$$

where $f^{[n]}$ represents the density function of $X_{1}+\cdots+X_{n}$. Бy Theorem 3.7 we know that $f^{[n]}(x)$ is $\mathrm{TP}_{r}$. Applying $(0.4)$ to (7.1), it follows that $h(x, \lambda)$ is $\operatorname{TP}_{r}$.

As an illustration of this result, we may identify $\lambda$ with a time parameter $t$ and consider for $N(t)$ a (Poisson) process. By virtue of Theorem 4.2, part (i), $g(n, t)=\operatorname{Pr}\{N(t)=n\}$ is $\mathrm{TP}_{r}$. The previous conclusion now asserts that

$$
h(x, \lambda)=e^{-\lambda} \sum_{n=0}^{\infty} f^{[n]}(x) \frac{\lambda^{n}}{n !} \text { is } \operatorname{TP}_{\infty} \text { for } x>0 \text { and } \lambda>0 .
$$

The concept of a random number of observations for a stochastic process has been generalized to a continuous version called the principle of subordination. 
A process $\mathscr{P}_{1}$ with transition probability function $Q(t ; x, E)$ is said to be subordinate to a process $\mathscr{P}_{2}$ with transition probability function $P(t ; x, E)$ if there exists a transition density $\gamma(t, u)$ of a positive infinitely divisible process such that

$$
Q(t ; x, E)=\int_{0}^{\infty} P(u ; x, E) \gamma(t, u) d u .
$$

Roughly speaking, the process $\mathscr{P}_{1}$ arises from $\mathscr{P}_{2}$ by a random change of time scale (see [2, Chapter 4] for a discussion of this concept and its ramifications). If $P(u ; x, E)$ has a density function $p(u ; x, y)$, then so does $Q(t ; x, E)$ and

$$
q(t ; x, y)=\int_{0}^{\infty} p(u ; x, y) \gamma(t, u) d u .
$$

If $p(u ; x, y)$ is $\mathrm{TP}_{r}$ in the variables $u$ and $y$ and $\gamma(t, u)$ is $\mathrm{TP}_{r}$, then an application of (0.4) to (7.3) yields that $q(t ; x, y)$ is $\mathrm{TP}_{r}$ in the variables $t$ and $y$. In particular, we have the following theorem.

THEOREM 7.1. Let $\mathscr{P}_{2}$ be a $\mathrm{TP}_{r}$ Markoff process of non-negative drift with transition density $p(t ; x, y)$ and let $\gamma(t, u)$ be the transition density of a $\mathrm{TP}_{r}$ infinitely divisible process of non-negative drift.

(i) Then the subordinate process $\mathscr{P}_{1}$ with transition density

$$
q(t ; x, y)=\int_{0}^{\infty} p(u ; x, y) \gamma(t, u) d u
$$

is $\mathrm{TP}_{r}$ for $t>0$ and $y \geqq x$.

(ii) If $\mathscr{P}_{2}$ is symmetric and $\mathrm{TP}_{r}$, then $q(t ; 0, y)$ is $\mathrm{TP}_{r}$ for $t>0$ and $y \geqq 0$.

The last assertion is proved by appealing to Theorem 6.5 and (0.4). We shall record some illustrations of this result in $\$ 9$.

2. Theorem 7.1 describes a class of transformations on $\mathbf{T P}_{r}$ functions that produces new $\mathrm{TP}_{\boldsymbol{r}}$ functions. Further interesting examples of sign-regular functions are obtained by computing Laplace transformations and generating functions of first-passage probabilities of the kind introduced in $\S \S 2-6$. Thus, if $q(t, x)$ is bounded and $\mathrm{TP}_{r}$ for $t>0$ and $x>0$ then

$$
W(x, s)=\int_{0}^{\infty} e^{-s t} q(t, x) d t, \quad s>0,
$$

is $\mathrm{RR}_{r}$ for $s>0$ and $x>0$. This follows by applying (0.4) to (7.4) using the fact that $K(s, t)=e^{-s t}$ is $\mathrm{RR}_{\infty}$ for $-\infty<s, t<\infty$.

In the discrete analog, let $q(n, x)$ be bounded and $\mathrm{TP}_{r}$ for $n=0,1, \cdots$ and $x>0$; then

$$
W(x, s)=\sum_{n=0}^{\infty} s^{n} q(n, x), \quad 0<s<1,
$$

is $\mathrm{TP}_{r}$ for $0<s<1$ and $x>0$. 
Other applications of the transformation law (0.3) are available. We mention without entering into details the Bessel transformation of imaginary argument and the Stieltjes transform. Several consequences and extensions of assertions (7.4) and (7.5) are discussed in $\$ \S 9$ and 11. The main applications concern Tchebycheff systems of functions.

3. In the spirit of the preceding analysis we investigate the nature of the determinantal inequalities of the function $c(t+s)$ where

$$
c(t)=\int_{0}^{\infty} \phi(t, x) f(x) d x, \quad t>0 .
$$

We assume that $f(x+y)$ is sign regular (either $\mathrm{TP}_{r}$ or $\mathrm{RR}_{r}$ ) and that $\phi(t, x)$ is TP, for $t>0$ and $x>0$ and obeys the semi-group property

$$
\phi(t+s, x)=\int_{0}^{x} \phi(t, \xi) \phi(s, x-\xi) d \xi .
$$

Under these conditions we prove that $c(t+s)$ is $\operatorname{TP}_{r}\left(\mathrm{R}_{r}\right)$ according as $f(x+y)$ is $\operatorname{TP}_{r}\left(R_{r}\right)$. To this end, we examine the expression

$$
\begin{aligned}
c(t+s) & =\int_{0}^{\infty} \phi(t+s, x) f(x) d x \\
& =\int_{0}^{\infty} \phi(t, \xi) \quad \int_{0}^{\infty} \phi(s, u) f(u+\xi) d u d \xi .
\end{aligned}
$$

The last identity results by applying (7.7), interchanging orders of integration and then making an obvious change of variable. Invoking the hypothesis with the help of $(0.4)$ we infer that the function

$$
\Psi(s, \xi)=\int_{0}^{\infty} \phi(s, u) f(u+\xi) d u
$$

is sign regular. Moreover, the determinants of a given order formed from the kernel $\Psi(s, \xi)$ are of the same sign as the determinants of the same order formed from $f(u+\xi)$. Now, (7.8) can be written in the form:

$$
c(t+s)=\int_{0}^{\infty} \phi(t, \xi) \Psi(s, \xi) d \xi
$$

Another application of (0.4) yields that $c(t+s)$ is appropriately $\mathrm{TP}_{r}$ or $\mathrm{RR}_{r}$.

The above arguments hold without change in the case that the variable $t$ is confined to vary over the set of positive integers. We summarize the discussion in the statement of the following theorem.

THEOREM 7.2. Let $f(x+y)$ be $\operatorname{TP}_{r}\left(\mathrm{RR}_{r}\right)$ for $x>0$ and $y>0$. Suppose $\phi(t, x)$ is $\mathrm{TP}_{r}$ for $x>0$ and $t>0$ (or $\left.t=1,2,3, \cdots\right)$ and satisfies (7.7). Let $c(t)$ 
be defined by (7.6). Then $c(t+s)$ is $\mathrm{TP}_{r}\left(\mathrm{R}_{r}\right)$ for $t, s>0$ (for $t, s=1,2, \cdots$ in the discrete case).

This theorem enables us to deduce various moment inequalities for $\mathrm{PF}_{r}$ densities associated with non-negative random variables. We indicate such applications and others in the last part of $\$ 11$.

The hypotheses of Theorem 7.2 are satisfied in the following circumstances:

Let $f(x)$ be a $\mathrm{PF}$, density function of a non-negative random variable.

(a) Let $\phi(n, \xi)$ be the density function of the sum $X_{1}+X_{2}+\cdots+X_{n}$ where $X_{i}$ are positive identically and independently distributed random variables whose density function $\phi(\xi)$ is $\mathrm{PF}_{r}$. The fact that $\phi(n, \xi)$ is $\mathrm{TP}$, for $n \geqq 1$ and $\xi>0$ follows from Theorem 3.7, part (i).

(b) Let

$$
\phi(t, \xi)=\left\{\begin{array}{cc}
\frac{\xi^{t-1}}{\Gamma(t)}, & \xi>0, \\
0, & \xi \leqq 0 .
\end{array}\right.
$$

Relation (7.7) and the TP, property may be verified by direct calculation.

(c) Let $\phi(t, \xi)$ denote the density function of a stable process $X(t)$ with nonnegative drift whose Laplace transform is $\exp \left(-t s^{1 / k}\right), s>0(k=$ a fixed positive integer $\geqq 2)$. It is shown in $\S 9$ that $\phi(t, \xi)$ is $\mathrm{TP}_{\infty}$ for $t, \xi>0$. Property (7.7) emerges from the fact that $X(t)$ is a homogeneous process with non-negative drift.

4. We close this section with an application of the preceding theory to the case of the classical discrete-time branching processes. A branching process may be characterized in the following manner. Suppose an organism at the end of its lifetime produces a random number $\xi$ of offspring with probability distribution

$$
\operatorname{Pr}\{\xi=k\}=a_{k} \quad(k=0,1,2, \cdots),
$$

where, as usual, $a_{k} \geqq 0$ and

$$
\sum_{k=0}^{\infty} a_{k}=1
$$

We assume that all offspring act independently of each other, and have the same distribution of lifetime and progeny. The process $X(n)$ describing the population size in the $n$th generation is a temporally homogeneous Markoff chain whose transition matrix is

$$
\begin{aligned}
P_{n}(i, j) & =\operatorname{Pr}\{X(n+1)=j \mid X(n)=i\} \\
& =\operatorname{Pr}\left\{\xi_{1}+\xi_{2}+\cdots+\xi_{i}=j\right\}
\end{aligned}
$$


where the $\xi$ 's are independent observations of a random variable with the probability law (7.9).

We consider now the special case of a pure-growth branching process, i.e., a process in which $a_{0}=0(\mathscr{P}$ has non-negative drift), and we assume that the function

$$
G(k, l)= \begin{cases}a_{k-l}, & k>l, \\ 0, & k \leqq l\end{cases}
$$

is $\mathrm{TP}_{r}$. In other words, $a_{k}$ is a Pólya frequency sequence of order $r$. The transition function (7.10) is plainly the probability function of an $i$-fold convolution of the law (7.9), and hence by Theorem 2.5 is $\mathrm{TP}_{r}$. The hypotheses of Corollary 2.1 are now established and we conclude that

$$
P^{(n)}(1, j)=\operatorname{Pr}\{X(n)=j \mid X(0)=1\}
$$

is $\operatorname{TP}_{r}$ for $n \geqq 0$ and $j \geqq 1$. The generating function of $X(n)$, viz.,

$$
g_{n}(z)=\sum_{j=1}^{\infty} P^{(n)}(1, j) z_{j}, \quad 0<z<1,
$$

satisfies the functional equation

$$
g_{n}(z)=g\left(g_{n-1}(z)\right),
$$

Now from the statement of (7.5) we deduce the interesting property that $g_{n}(z)$ is TP in the variables $n \geqq 1$ and $0<z<1$ provided $a_{k}$ is $\mathrm{PF}_{r}$.

8. Probabilistic examples and applications. In this section we discuss a series of examples and applications of the previous theorems which are motivated mainly by various probability models.

1. Consider a temporally and spatially homogeneous Markoff chain $\mathscr{P}$ on the integers with transition probability matrix

$$
P(i, j)=\left\{\begin{array}{ll}
p, & j=i+1, \\
q, & j=i, \\
0, & \text { otherwise, }
\end{array} \quad p, q>0\right.
$$

and $p+q=1$. Clearly $\mathscr{P}$ is $\mathrm{TP}_{\infty}$ and we conclude by Theorem 2.5 that the binomial density

$$
f(n, k)=P^{n}(0, k)=\left(\begin{array}{l}
n \\
k
\end{array}\right) p^{k} q^{n-k} \text { is } \mathrm{TP}_{\infty}, \quad n, k \geqq 0 .
$$

In particular, $\left(\begin{array}{l}n \\ k\end{array}\right)$ is $\mathrm{TP}_{\infty}$.

2. Let $\mathscr{P}$ be a spatially homogeneous nontemporally homogeneous Markoff chain where the transition probability matrix at the $m$ th transition is of the special form 


$$
P_{m}(i, j)=\left\{\begin{array}{cl}
\frac{p^{m}}{1+p^{m}}, & j=i+1, \\
\frac{1}{1+p^{m}}, & j=i, \\
0, & \text { otherwise. }
\end{array}\right.
$$

But by virtue of Theorem 2.5 we have that

is $\mathrm{TP}_{\infty}$ where, by definition,

$$
g(n, k)=P^{n}(0, k)=\frac{\left[\begin{array}{l}
n \\
k
\end{array}\right] p^{\left(k^{2}+k\right) / 2}}{\prod_{i=1}^{n}\left(1+p^{i}\right)} \quad n, k \geqq 0
$$

$$
\left[\begin{array}{l}
n \\
k
\end{array}\right]= \begin{cases}\frac{\left(1-p^{n}\right)\left(1-p^{n-1}\right) \cdots\left(1-p^{n-k+1}\right)}{\left(1-p^{k}\right)\left(1-p^{k-1}\right) \cdots(1-p)} & \text { for } k \leqq n \\
& \text { otherwise. }\end{cases}
$$

The quantity (8.1) is known as the Heine coefficient and can be construed as a generalization of the binomial coefficient since

(see [10]).

$$
\lim _{p \rightarrow 1}\left[\begin{array}{l}
n \\
k
\end{array}\right]=\left(\begin{array}{l}
n \\
k
\end{array}\right)
$$

3. Consider a spatially and temporally homogeneous Markoff chain where

$$
P(i, j)=\left\{\begin{array}{ll}
q^{j-i} p, & j \geqq i, \\
0, & \text { otherwise. }
\end{array} \quad(0<p<1, p+q=1),\right.
$$

We may verify directly that $\mathscr{P}$ is $\mathrm{TP}_{\infty}$. Hence by Theorem 2.5 we deduce that

$$
P^{n}(0, k)=\left(\begin{array}{c}
n+k-1 \\
k
\end{array}\right) p^{n} q^{k} \text { is } \mathrm{TP}_{\infty} \quad n, k \geqq 0 .
$$

4. Example 3 can be generalized in the manner in which example 1 was generalized in example 2 . Let $\mathscr{P}$ be a spatially homogeneous Markoff chain whose $m$ th step transition probability matrix is of the form

$$
P_{m}(i, j)=\left\{\begin{array}{ll}
q^{m(j-i)}\left(1-q^{m}\right), & j \geqq i, \\
0, & \text { otherwise. }
\end{array} \quad(0<q<1),\right.
$$


A direct verification yields that $\mathscr{P}$ is $\mathrm{TP}_{\infty}$. Using the Heine hypergeometric relation $[10$, p. 8$]$

$$
\frac{1}{\prod_{i=1}^{r+1}\left(1-q^{i} s\right)}=1+\sum_{k=1}^{\infty} \frac{[r+1][r+2] \cdots[r+k]}{[1][2] \cdots[k]} s^{k}
$$

where $[m]=\left(1-q^{m}\right) /(1-q)$ by definition, we obtain that

$$
P^{n}(0, k)=q^{k}\left\{\prod_{i=1}^{n}\left(1-q^{i}\right)\right\} \frac{[n][n+1] \cdots[n+k-1]}{[1][2] \cdots[k]}
$$

is $\mathrm{TP}_{\infty}$.

5. We may use Theorem 2.5 for a model of coin tossing and deduce some applications to analysis pertaining to zeros of polynomials. Let

$$
P_{n}(i, j)=\left\{\begin{array}{ll}
p_{n}, & j=i+1, \\
q_{n}, & j=i, \\
0, & \text { otherwise. }
\end{array} \quad\left(p_{n}+q_{n}=1,0<p_{n}<1\right)\right.
$$

The process $\mathscr{P}$ is a Markoff chain of non-negative drift whose realizations are the outcomes of independent coin tosses where the probability of success at the $n$th toss is $p_{n}$. By Theorem 2.5 we know that the $n$-step transition matrix $P^{n}(0, j)$ is $\mathrm{TP}_{\infty}$ for $n \geqq 0$ and $j \geqq 0$. The associated generating function has the form

$$
\begin{aligned}
\sum_{j=0}^{\infty} P^{n}(0, j) z^{j} & =\left(q_{1} q_{2} \cdots q_{n}\right) \prod_{i=1}^{n}\left(z+\frac{p_{i}}{q_{i}}\right)=q_{1} q_{2} \cdots q_{n} \cdot f_{n}(z), \quad n \geqq 1, \\
f_{0}(z) & =1 .
\end{aligned}
$$

We infer by (7.5) that $f_{n}(z)$ is $\operatorname{TP}_{\infty}$ in the variables $z>0$ and $n>0$. Actually further scrutiny of the analysis shows more; namely that $f_{n}(z)$ is $\operatorname{ETP}(z)$. It follows by virtue of the variation diminishing property that

$$
\begin{aligned}
P(x)=\sum_{m=0}^{n} c_{m} f_{m}(x)=c_{0} & +c_{1}\left(x+a_{1}\right)+c_{2}\left(x+a_{1}\right)\left(x+a_{2}\right) \\
& +\cdots+c_{n}\left(x+a_{1}\right)\left(x+a_{2}\right) \cdots\left(x+a_{n}\right) \\
& \left(a_{i}=p_{i} / q_{i}, i=1,2, \cdots, n\right)
\end{aligned}
$$

possesses, in the interval $[0, \infty)$, a number of zeros counting multiplicities, not in excess of the number of sign changes of the sequence $c_{0}, c_{1}, c_{2}, \cdots, c_{n}$. Here, $a_{1}, a_{2}, \cdots, a_{n}$ can denote any set of positive numbers.

Various generalizations which exploit the other theorems of $\S \S 2-6$ suggest themselves. We carry out one further example. Let $\mathscr{P}$ be the generalized process (see the following paragraph) 


$$
P_{n}(i, j)= \begin{cases}a_{n}^{j-i}, & j>i \\ 0, & \text { otherwise }\end{cases}
$$

where $a_{n}$ are arbitrary positive numbers and the row sum need not equal 1 and may even be nonsummable.

At this time it is useful to point out that many of the results of $\S \S 2-5$ do not depend on the probability formulation although significantly this served as the motivation. It suffices to observe that under the proper formal definitions (e.g. see the footnote on this page) the renewal relations like (2.2), etc., persist and all the arguments carry over without change and the corresponding conclusions hold under proper interpretation.

Here $\mathscr{P}$ behaves like a spatially homogeneous nontemporally homogeneous $\mathrm{TP}_{\infty}$ Markoff chain with non-negative $\operatorname{drift}\left({ }^{9}\right)$. Theorem 2.5 implies that $P^{n}(0, j)$ is $\mathrm{TP}_{\infty}$. Its generating functions exist for $z$ sufficiently small and has the form

$$
f(z)=\prod_{i=1}^{n}\left(\frac{z a_{i}}{1-z a_{i}}\right)=\frac{z^{n}}{\left(b_{1}-z\right)\left(b_{2}-z\right) \cdots\left(b_{n}-z\right)}
$$

with $b_{i}=1 / a_{i}(i=1,2, \cdots, n)$.

Now we consider the transformation $C_{j}=\sum_{k=0}^{n} D_{k} P^{k}(0, j), j=0,1, \cdots$. This is variation diminishing since $P^{k}(0, j)$ is $\mathrm{TP}_{\infty}$. The transformation can also be formally represented as follows: Taking generating functions

$$
\sum_{j=0}^{\infty} C_{j} z^{j}=D_{0}+D_{1} \frac{z}{b_{1}-z}+\frac{D_{2} z^{2}}{\left(b_{1}-z\right)\left(b_{2}-z\right)}+\cdots+\frac{D_{n} z^{n}}{\prod_{i=1}^{n}\left(b_{i}-z\right)}
$$

which is valid for $z$ sufficiently small. Our result affirms that if $C_{i}$ and $D_{j}$ are related according to $\left(^{*}\right)$ then $V(D) \geqq V(C)$ where $V(D)$ designates the number of sign changes of the sequence $D_{0}, D_{1}, \cdots, D_{n}$ and similarly for $V(C)$. The reader may find it of interest to compare this analysis with that of Pólya-Szegö II, problem 35, whose technique is rather complicated. The present approach shows clearly the general setting of the result.

6. Consider a temporally homogeneous Markoff chain on the non-negative integers with transition matrix

( $\left.{ }^{9}\right)$ In this case $\mathrm{P}^{n}(0, j)$ is not interpreted as a probability function but we operate with it as if it is. The formal definition suitable for our purposes is

$$
P^{n}(0, j)=\sum_{0 \leqq i_{1} \leqq i_{2} \leqq \ldots \leqq i_{k} \leqq j} P_{1}\left(0, i_{1}\right) P_{2}\left(i_{1}, i_{2}\right) \cdots P_{h}\left(i_{k}, j\right) .
$$

In the case that $P_{m}\left(i_{1}, i_{2}\right)$ is a bonafide probability transition function the quantity $P^{n}(0, j)$ then certainly agrees with the $n$-step probability transition function. We postulate that $P^{n}(0, j)$ is well defined and finite, i.e., the series converges absolutely. 


$$
\begin{aligned}
& P(i, j)=\left\{\begin{array}{cl}
c, & j=i+1, \\
b, & j=i, \\
a, & j=i-1, \\
0, & \text { otherwise, }
\end{array} \quad i=1,2, \cdots,\right. \\
& P_{00}=b+a, \quad P_{0_{1}}=c .
\end{aligned}
$$

We will determine the precise conditions on the constants $a, b, c$ which assure that (8.2) is totally positive of order $r$. To this end we observe that the process is $\mathrm{TP}_{r}$ if and only if the determinant of the $q \times q$ matrix

$$
A_{q}=\left(\begin{array}{cccccc}
b & c & & & & \\
a & b & c & & & \\
& a & b & c & & \\
& \cdot & \cdot & \cdot & & \\
& & \cdot & \cdot & \cdot & \\
& & & \cdot & \cdot & \cdot \\
& & & a & b & c \\
& & & & a & b
\end{array}\right) .
$$

is non-negative for $q=1,2, \cdots, r$. The determinant $\left|A_{q}\right|$ is evaluated by observing that $D_{q}(\lambda)=\left|A_{q}-\lambda I\right|(I=$ identity matrix $)$ obeys the recursion law

$$
D_{q+1}(\lambda)=(b-\lambda) D_{q}(\lambda)-a c D_{q-1}(\lambda), \quad r \geqq 0,
$$

provided $D_{-1}(\lambda)=0, D_{0}(\lambda)=1$. Some elementary transformations executed on (8.3) lead to a recognizable recurrence formula. Then, we can conclude that

where

$$
D_{q}(\lambda)=(\sqrt{ }(a c))^{q} Q_{q}\left(\frac{b-\lambda}{2 \sqrt{ }(a c)}\right)
$$

$$
Q_{q}(x)=\frac{\sin (q+1) \theta}{\sin \theta}, \quad \cos \theta=x,
$$

stands for the classical Tchebycheff polynomials of the second kind.

If $b / 2 \sqrt{ }(a c) \geqq 1$ then it is simple to prove generally that $D_{q}(0)>0$. Therefore, we consider only the case that $b / 2 \sqrt{ }(a c)<1$. It is clear that $\left|A_{q}\right|=D_{q}(0) \geqq 0$ $(1 \leqq q \leqq r)$ if and only if

$$
|\theta| \leqq \frac{\pi}{r+1} \text { where } \cos \theta=\frac{b}{2 \sqrt{ }(a c)} .
$$

In particular, $\mathscr{P}$ is $\mathrm{TP}_{2}$ if and only if $0<\pi / 3$ or equivalently $b / 2 \sqrt{ }(a c) \geqq \frac{1}{2}$ $\left(b^{2} \geqq a c\right)$. Also $\mathscr{P}$ is $\mathrm{TP}_{3}$ if and only if $\theta<\pi / 4$ or what is the same $(b / 2 \sqrt{ }(a c))>\sqrt{ } 2 / 2$ $\left(b^{2} \geqq 2 a c\right)$. In general, $\mathscr{P}$ is $\operatorname{TP}_{r}$ if and only if $(b / 2 \sqrt{ }(a c)) \geqq \cos (\pi / r+1)$.

Let $u(s)=a+b s+c s^{2}$ and construct $P^{n}(0, k)$ as the coefficients of the power series

$$
[u(s)]^{n}=\sum_{k=0}^{\infty} P^{(n)}(0, k) s^{k}
$$

Then provided $(b / 2 \sqrt{ }(a c)) \geqq \cos (\pi / r+1)$ it follows that $P^{n}(0, k)$ is $\operatorname{TP}_{r}$. 
7. Consider the noncentral chi square density function

$$
f(x)= \begin{cases}e^{-\lambda} \sum_{n=0}^{\infty} \frac{\lambda^{n}}{n !} \frac{x^{n+\alpha-1}}{\Gamma(n+\alpha)} e^{-x}, & x \geqq 0, \\ 0, & x<0 .\end{cases}
$$

This density can be expressed in terms of IBessel functions; viz., $f(x)=e^{-\lambda x}(x / \lambda)^{(\alpha-1) / 2} I_{\alpha-1}(\sqrt{ }(2 \lambda x))$ for $x>0$. Let $\mathscr{P}$ represent the spatially homogeneous discrete-time process $X(n)$ where $X(0)=0, X(n)=\xi_{1}+\xi_{2}+\cdots+\xi_{i i}$. $n \geqq 1$ and $\xi_{i}$ are independently distributed according to the distribution function (8.4) with possibly different choices of $\alpha$ associated with each term in the summand. We prove in $\S 9$ that $(8.4)$ is $\mathrm{PF}_{r}$ provided $\alpha>r-1$. An easy calculation via the Laplace transform of (8.4) yields the distribution function of $X(n)$ :

$$
p(n, x)=e^{-\lambda n} \sum_{l=0}^{\infty} \frac{\lambda^{l} n^{l}}{l !} \frac{x^{l+\alpha_{1}+\alpha_{2}+\ldots+\alpha_{n}-1}}{\Gamma\left(l+\alpha_{1}+\alpha_{2}+\cdots+\alpha_{n}\right)} e^{-x} .
$$

By Theorem 3.7, we have that (8.5) is TP, provided $\alpha_{i}>r-1$ for all $i$.

8. Let $P_{i j}(t)$ represent the transition function of a pure birth process; then by Theorem 4.2 we know that $P_{i j}(t)$ is $\mathrm{TP}_{\infty}$ with respect to the variables $t>0$ and $j \geqq i$.

The following application is of some interest. Let $X(t)$ denote a pure birth process whose waiting time in the $k$ th state follows an exponential distribution of parameter $\lambda_{k}$. The Laplace transform of the first-passage time from state 0 to state $k+1$ is of the form

$$
\Phi_{k}(s)=\frac{1}{\left(s+\lambda_{0}\right)\left(s+\lambda_{1}\right) \cdots\left(s+\lambda_{k}\right)}, \quad k=0,1, \cdots, s>0 .
$$

By Theorem 4.2, part (ii), and (7.4) we deduce that $\Phi_{k}(s)$ is $R R_{\infty}$. It follows, in particular, that $\Phi_{k}(s)$ constitute a Tchebycheff family of functions generalizing those considered by Pólya-Szegö (problem 84, Chapter 6).

9. Consider a stationary birth-and-death process $\mathscr{P}$ on the non-negative integers. It is proved in [16] that the transition probability function $P_{i j}(t)$ possesses an integral representation of the form

$$
P_{i j}(t)=\pi_{j} \int_{0}^{\infty} e^{-x t} Q_{i}(x) Q_{j}(x) d \psi(x)
$$

where $Q_{i}(x)$ comprise an appropriate system of polynomials properly normalized and orthogonal with respect to the measure $\psi$ which concentrates its mass on the non-negative axis. Here $\pi_{j}$ are suitable constants depending on the birth and death rates of the process. The polynomials $Q_{i}(x)$ are defined recursively by means of the relations 


$$
\begin{gathered}
-x Q_{k}(x)=-\left(\lambda_{n}+\mu_{n}\right) Q_{n}(x)+\lambda_{n} Q_{n+1}(x)+\mu_{n} Q_{n-1}(x), \quad n \geqq 0, \\
Q_{-1}(x) \equiv 0 \text { and } Q_{0}(x) \equiv 1,
\end{gathered}
$$

where $\lambda_{n}$ and $\mu_{n}$ designate the infinitesimal birth and death rates respectively corresponding to state $n$ and

$$
\pi_{n}=\frac{\lambda_{0} \lambda_{1} \cdots \lambda_{n-1}}{\mu_{1} \mu_{2} \cdots \mu_{n}}, \quad n \geqq 1, \pi_{0}=1 .
$$

These parameters satisfy the conditions $\lambda_{n}>0, \mu_{n}>0$ for $n \geqq 0$ with the exception that $\mu_{0} \geqq 0$. We refer the reader to [16] and [17] for a thorough discussion of birth-and-death processes.

It was pointed out earlier (see $\S 4$ ) that $\mathscr{P}$ is $\mathrm{TP}_{\infty}$. Now according to Theorem 4.3, part (i), we have the result that

$$
P_{0 j}(t)=\pi_{j} \int_{0}^{\infty} e^{-x t} Q_{j}(x) d \psi(x)
$$

is $\mathrm{TP}_{\infty}$.

In particular, invoking (0.4), we secure the relation

$$
\int_{0 \leqq x_{1}<x_{2}<\ldots<x_{n}} \operatorname{det}\left[\exp \left(-x_{k} t_{i}\right)\right] \operatorname{det}\left[Q_{j_{v}}\left(x_{k}\right)\right] d \psi\left(x_{1}\right) d \psi\left(x_{2}\right) \cdots d \psi\left(x_{n}\right) \geqq 0
$$

for any collection of $t_{i}$ and $j_{v}$ arranged so that $0<t_{1}<t_{2}<\cdots<t_{n}$ and $0 \leqq j_{1}<j_{2}<\cdots<j_{n}$, respectively.

Theorem 4.3, part (iv), shows that $P_{00}(t+s)$ is $\mathrm{TP}_{\infty}$ for $t, s>0$, where

$$
P_{00}(t)=\int_{0}^{\infty} e^{-x t} d \psi(x)
$$

Actually this fact may be verified directly from the integral representation using (0.4) combined with the fact that $e^{-x t}$ is $\mathrm{RR}_{\infty}$ for $x$ and $t$ real.

Other examples of total positive and sign-regular kernels connected with birth-and-death processes can be generated by considering the distribution functions of the first-passage time between states. We present an application to the theory of polynomials.

Let $f_{i j_{0}}(t)$ denote the density function of the time of first passage from state $i$ to state $j_{0}>i$. Theorem 4.1, part (i), implies that $f_{i j}(t)$ is $\mathbf{R R}_{\infty}$ for $t>0$ and $i$ $\left(i<j_{0}\right)$. The Laplace transform of $f_{i j}(t)$ is explicitly evaluated in [22] (see also [21]) and shown there to be $Q_{i}(-s) / Q_{j_{0}}(-s)$ where $Q_{i}$ is the polynomial system determined in (8.7). But, as noted in (7.4) the Laplace transformation takes an $\mathbf{R}_{r}$ function into a total positive function of the same order. In particular, 
we conclude that $Q_{i}(-s)$ is $\mathrm{TP}_{\infty}$ for $s>0$ and $i \geqq 0$. A direct, rather more complicated induction proof shows that $Q_{i}(-s)$ is $\operatorname{ETP}_{\infty}(s)$ [21, §1].

We now develop a related result. It is shown in [16, equation (3.21)] that the Laplace transform of (8.8) is

$$
\begin{aligned}
R_{j}(-s) & =Q_{j}^{(0)}(-s) \pi_{j}+\pi_{j} Q_{j}(-s) R(s) \\
& =\pi_{j} Q_{j}(-s) \sum_{k=j}^{\infty} \frac{1}{\lambda_{k} \pi_{k} Q_{k}(-s) Q_{k+1}(-s)}, \quad j \geqq 0, s>0
\end{aligned}
$$

where $Q_{j}$ denotes the polynomial system determined in (8.7) and $Q_{j}^{(0)}$ describes a polynomial system defined by the same recurrence formula under the new initial conditions $Q_{-1}^{(0)}(-s) \equiv 1 / \lambda_{0}, Q_{0}^{(0)}(s) \equiv 0$.

In other words, $Q_{j}$ and $Q_{j}^{0}$ are independent solutions of (8.7) subject to the initial conditions as indicated. Here,

$$
R(s)=\int_{0}^{\infty} \frac{d \psi}{x+s}
$$

where $\psi$ is the measure of the process (see (8.6)).

By virtue of (7.4) and the statement of (8.8) we conclude that $R_{j}(-s)$ is $\mathbf{R} \mathbf{R}_{\infty}$ for $j \geqq 0$ and $s>0$. This result derives further interest due to the fact that $Q_{j}(-s)$ is $\mathrm{TP}_{\infty}$ as contrasted to the statement that $R_{j}(-s)$ is $\mathbf{R R}_{\infty}$. Thus, in particular, $Q_{j}(-s)$ is increasing in $j$ while $R_{j}(-s) / R_{\jmath}(-\varepsilon)$ is decreasing in $j$, for $s>\varepsilon>0$.

10. Consider the process $\mathscr{P}$ whose sample functions $X(t)$ represent the monetary gain of a sequence of coin tossings performed at random times with success and failure equally likely. Specifically, let $X(t)$ be the sum of a random number, $N(t)$, of independent identically distributed Bernoulli variables with distribution $\operatorname{Pr}\{\xi=+1\}=\operatorname{Pr}\{\xi=-1\}=\frac{1}{2}$, where $N(t)$ is a sample function of a Poisson process with parameter $\lambda$. This process also arises in a fundamental way in the theory of insurance risk. We may regard the process equivalently as an extended homogeneous birth-and-death process whose state space coincides with the full set of integers with constant birth and death rate $\lambda / 2$ independent of the state of the process. Thus $\mathscr{P}$ is $\mathrm{TP}_{\infty}$ since the transition matrix of any birth-and-death process is $\mathrm{TP}_{\infty}$.

We are interested in the random variable

$$
Z(t)=\max _{0 \leqq \tau \leqq t} X(\tau)
$$

related to the ruin probability in the theory of collective insurance risk.

The quantity $\operatorname{Pr}\{Z(t)<n\}$ is equal to the probability that the first passage into state $n$ starting from state 0 occurs at a time later than $t$. In symbols, 


$$
\operatorname{Pr}\{Z(t)<n\}=1-F_{0 n}(t)
$$

where $F_{0 n}(t)$ denotes the distribution function of the random variable $T_{0 n}=$ the time of first passage from state 0 to state $n$. This quantity has been explicitly caculated [1];

$$
f_{0 n}(t)=F_{0 n}^{\prime}(t)=\frac{n e^{-\lambda t} I_{n}(\lambda t)}{t}
$$

where $I_{\alpha}$ denotes the usual Bessel function with imaginary argument. By Theorem 4.1, part (iii), we conclude that $I_{n}(\lambda t)$ is $\mathrm{TP}_{\infty}$ in the variables $n \geqq 1$ and $t>0$.

Taking account of the homogeneous nature of the process we obtain in the standard way the renewal equation

$$
f_{0, n+m}(t)=\int_{0}^{t} f_{0, n}(\xi) f_{0, m}(t-\xi) d \xi .
$$

Applying (0.4) and recognizing the fact that $f_{0 n}(t)$ is $\mathrm{TP}_{\infty}$ for $n \geqq 1$ and $t>0$ we deduce that $f_{0, n+m}(t)$ is $\mathbf{R R}_{\infty}$ in the variables $n \geqq 1$ and $m \geqq 1$ when $t$ is fixed.

Finally we point out for completeness of this discussion that

$$
e^{\lambda \sqrt{ }(t+s)}(t+s)^{(n+1 / 2)} f_{0 n}(\sqrt{ }(t+s))
$$

is $\mathrm{SRR}_{n+1}$ with respect to the variables, $t, s>0$. This fact will be derived by direct analytical arguments in the following section.

11. Let $\mathscr{P}$ denote the Brownian motion process whose transition density function is the familiar normallaw

$$
p(t ; x, y)=\frac{1}{\sqrt{ }(2 \pi t)} \exp \left[-\frac{1}{2 t}(x-y)^{2}\right] .
$$

Let $T(x)$ represent the time of first passage from the interval $(-\infty, x), x>0$ under the condition that the initial position is the origin. The family of random variables $T(x)$ with $x$ regarded as a parameter is the well-known stable process of order $1 / 2$ of positive drift. Explicitly, we have the density function

$$
V_{x}(t)=\operatorname{Pr}(T(x) \leqq t)=\frac{x}{\sqrt{ }(4 \pi)} \int_{0}^{t} \frac{1}{\tau^{3 / 2}} \exp \left(-\frac{x^{2}}{4 \tau}\right) d \tau .
$$

By Theorem 5.1, part (ii), $V_{x}^{\prime}(t)$ is $\mathrm{TP}_{\infty}$ for $t>0$ and $x>0$. Actually this property can be verified by direct calculation. However, the following considerations lead to functions of less obvious sign-regular character. Let the random variables $U(a, x)$ denote the time that a Brownian particle first leaves the open interval $(-a, a)$ where the starting position is $x(0 \leqq x<a)$. Let $f_{a}(t ; x)$ designate the density function of $U(a, x)$ which exists. In fact, 


$$
f_{a}(t ; x)
$$

$$
=\frac{\pi}{a^{2}} \sum_{j=0}^{\infty}(-1)^{j}\left(j+\frac{1}{2}\right) \cos \left[\left(j+\frac{1}{2}\right) \frac{\pi x}{a}\right] \exp \left[-\left(j+\frac{1}{2}\right)^{2} \pi^{2} t / 2 a^{2}\right]
$$

(see [4]). We now prove that (8.14) is $R_{\infty}$ with respect to the variables $t>0$ and $x$ satisfying $0 \leqq x<a$. To this end, we consider the radial process $Y(t)$ of the one-dimensional Brownian motion. In other words $Y(t)=|X(t)|$ where $X(t)$ represents the classical linear Brownian motion. $Y(t)$ has continuous paths and defines a strong Markoff process, the properties being inherited from the $X(t)$ process. Consequently, the $Y(t)$ process on $0 \leqq y<\infty$ is $\operatorname{TP}_{\infty}$ by virtue of the main theorem of [20]. Starting from $x$ the time $T_{a}(x)$ of first reaching the position $a$ is clearly the random variable $U(a, x)$. The desired conclusion is a consequence of Theorem 5.1, part (i), applied to the random variable $T_{a}(x)$.

We next consider a Brownian motion process $X(t)$ conditioned so that $\max _{0 \leqq u \leqq t}|X(u)|<1$. The transition density has been calculated explicitly by Levy [27, p. 213];

$$
\begin{aligned}
& \operatorname{Pr}\left\{X(t)=x, \max _{0 \leqq u \leqq t}|X(u)|<1 \mid X(0)=0\right\} \\
& =\frac{1}{\sqrt{ }(2 \pi t)} \sum_{n=-\infty}^{\infty}\left\{\exp \left[-\frac{(x-4 n)^{2}}{2 t}\right]-\exp -\left[\frac{(x+4 n+2)^{2}}{2 t}\right]\right\} .
\end{aligned}
$$

Consider the absolute value process $|X(t)|=Z(t)$ subject to the same condition as in (8.15). We easily obtain from (8.15) for the $Z(t)$ process a transition density

$$
\operatorname{Pr}\left\{Z(t)=x, \max _{0 \leqq u \leqq t} Z(u)<1 \mid Z(0)=0\right\}
$$

$$
=\frac{1}{\sqrt{ }(2 \pi t)} \sum_{n=-\infty}^{\infty}(-1)^{n}\left\{\exp \left[-\frac{(x+2 n)^{2}}{2 t}\right]+\exp \left[-\frac{(x-2 n)^{2}}{2 t}\right]\right\}, \quad x>0 .
$$

Appealing to Theorem 5.2, we have that (8.16) is $\mathrm{TP}_{\infty}$ for $0 \leqq x<1$ and $t>0$.

In the same spirit we deduce that

$$
L(t+s)=\sqrt{\left(\frac{2}{\pi(t+s)}\right)} \sum_{n=-\infty}^{\infty}(-1)^{n} \exp \left(-\frac{4 n^{2}}{t+s}\right)
$$

is $\mathrm{TP}_{\infty}$ for $t>0$ and $s>0$. In fact

$$
L(t+s)=\operatorname{Pr}\left\{Z(t+s)=0, \max _{0 \leqq u \leqq t+s} Z(u)<1 \mid Z(0)=0\right\}
$$

and the last statement of Theorem 5.2 is relevant. 
Other interesting examples arise as probability functions associated with the radial process induced by the motion of a Brownian particle moving freely in $N$-dimensional space. Thus let $X(t)=\left(X_{1}(t), X_{2}(t), \cdots, X_{N}(t)\right)$ denote a Brownian motion process in $N$ dimensions and let

$$
R(t)=\sqrt{ }\left(X_{1}^{2}(t)+\cdots+X_{n}^{2}(t)\right)
$$

be its radial part.

It is shown in [20] that the process $R(t)$ is $\mathrm{TP}_{\infty}, 0<t<\infty$. Its transition density function is quite familiar and recorded explicitly in [18];

$$
p(t ; r, \rho)=\int_{0}^{\infty} e^{-\alpha^{2} t} U(\alpha r) U(\alpha \rho) d \mu(\alpha)
$$

where

$$
\begin{aligned}
& U(x)=\Gamma\left(\gamma+\frac{1}{2}\right)\left(\frac{x}{2}\right)^{1 / 2-\gamma} J_{\gamma-1 / 2}(x), \\
& \mu(x)=2^{-(\gamma+1 / 2)}\left[\Gamma\left(\gamma+\frac{3}{2}\right)\right]^{-1} x^{2 r+1}
\end{aligned}
$$

and $\gamma=(N-1) / 2$. The transition probability function is

$$
P(t ; \rho, E)=\int_{E} p(t ; \rho, r) d \mu(r) .
$$

By Theorem 5.2, we conclude that $p(t ; 0, \rho)$ is $\mathrm{TP}_{\infty}$ in the variables $t>0$ and $\rho>0$.

We may now impose restrictions on the paths. Let $Y(t)$ denote the value of the $R(t)$ process defined above starting from a position $<1$ and terminated whenever its value equals 1 . The transition density is

$$
p(t ; x, y)=\sum_{n=0}^{\infty} e^{-\lambda_{n} t} \phi_{n}(x) \phi_{n}(y) \omega_{n}
$$

where

$$
\phi_{n}(x)=x^{-(N-2) / 2} J_{(N-2) / 2}\left(x \sqrt{ } \lambda_{n}\right), \quad \omega_{n}=\left[\int_{0}^{1} \phi_{n}^{2}(x) x^{N-1} d x\right]^{-1}
$$

and $\lambda_{n}$ denotes the sequence of positive zeros of $J_{(N-2) / 2}(x)$. By Theorem 5.2 we obtain that $p(t ; 0, y)$ is $\mathrm{TP}_{\infty}$ in the variables $t>0$ and $0 \leqq y<1$.

12. We develop some examples involving general one-dimensional diffusion processes where the transition density function satisfies a linear parabolic differential equation. Under slight conditions it may be established that every diffusion process generates a strcng Markoff process with continuous path function. By virtue of the probability coincidence theorem [20] we know that the process is $\mathrm{TP}_{\infty}$ and so we are justified in applying the theorems of $\$ 5$. 
The Brownian motion in many respects serves as the prime example of a diffusion process. We proceed to discuss other rather classical examples in terms of the present context.

A. Consider the diffusion equation on $-\infty<x<\infty$

$$
\frac{\partial u}{\partial t}=\frac{\partial^{2} u}{\partial x^{2}}-2 x \frac{\partial u}{\partial x}
$$

which is the backward equation for a particle executing Brownian motion and attracted to the origin by a restoring force proportional to the distance from the origin. The density function of the process (known as the Ornstein-Uhlenbeck process) with respect to Lebesgue measure is

$$
p(t ; x, y)=\frac{1}{\sqrt{ } \pi \sqrt{ }\left(1-e^{-4 t}\right)} \exp \left[-\left(x e^{-2 t}-y\right)^{2} /\left(1-e^{-4 t}\right)\right]
$$

We may verify directly that $p(t, 0, y)$ is $\mathrm{TP}_{\infty}$ for $t>0$ and $y>0$ as it should be agreeing with Theorem 5.3.

Let $f_{a-a}(t, x)$ denote the density function of the random variable which measures the time elapsed during which a particle starting at $x(0 \leqq x<a)$ and undergoing the Ornstein-Uhlenbeck process first leaves the symmetric interval $[-a, a]$. According to Theorem 5.1, part (i), $f_{a,-a}(t, x)$ is $\mathrm{RR}_{\infty}$ in the variables $x$ and $t$ where $0 \leqq x<a$ and $t>0$. It appears very difficult, if not impossible, to express $f_{a,-a}(t, x)$ in terms of elementary functions. However, the Laplace transform $\hat{f}$ of $f_{-a, a}(t, x)$ can be determined; thus [4],

$$
\hat{f}=\exp \left(\frac{x^{2}}{2}-\frac{a^{2}}{2}\right)\left\{\frac{D_{-\lambda}(x)+D_{-\lambda}(-x)}{D_{-\lambda}(a)+D_{-\lambda}(-a)}\right\}
$$

where $\exp \left(x^{2} / 2\right) D_{-\lambda}(x)$ and $\exp \left(x^{2} / 2\right) D_{-\lambda}(-x)$ are two linearly independent solutions of

$$
w^{\prime \prime}-2 x w^{\prime}-\lambda w=0 .
$$

These functions are related to the classical Weber functions. Taking account of the statement following (7.4) we infer that (8.20) is $\mathrm{TP}_{\infty}$ for $\lambda>0$ and $0<x<a$.

B. We consider the diffusion process whose transition probability functions satisfy the (backward) diffusion equation

$$
\frac{\partial u}{\partial t}=\frac{1}{(1-x)^{\alpha}(1+x)^{\beta}} \frac{\partial}{\partial x}\left\{(1-x)^{\alpha+1}(1+x)^{\beta+1} \frac{\partial u}{\partial x}\right\}
$$

on the interval $-1<x<1$ with the boundary conditions

$$
\left.\left.(1-x)^{\alpha+1} \frac{\partial u}{\partial x}\right]_{x=1}=0, \quad(1+x)^{\beta+1} \frac{\partial u}{\partial x}\right]_{x=-1}=0 .
$$


Here $\alpha, \beta>-1$ are parameters and of particular interest is the ultraspherical case $\alpha=\beta=\gamma-1 / 2$.

An explicit representation of the transition density is

$$
p(t ; x, y)=\sum_{n=0}^{\infty} e^{-\lambda_{n} t} P_{n}(x) P_{n}(y) w_{n}, \quad-1<x, \quad y<1,
$$

where

$$
\begin{aligned}
\lambda_{n}= & n(n+\alpha+\beta+1), \\
& w_{n}=\frac{\Gamma(\beta+1)}{\Gamma(\alpha+1) \Gamma(\alpha+\beta+1)} \frac{\Gamma(n+\alpha+1) \Gamma(n+\alpha+\beta+1)}{\Gamma(n+\beta+1) \Gamma(n+1)} \quad \frac{2 n+\alpha+\beta+1}{\alpha+\beta+1}
\end{aligned}
$$

and

$$
P_{n}(x)=\frac{P_{n}^{\alpha, \beta}(x)}{P_{n}^{\alpha, \beta}(1)}
$$

are the Jacobi polynomials normalized as shown. Our notation conforms to that of [34]. The associated transition probability function is

$$
\begin{aligned}
P(t ; x, E) & =\int p(t ; x y) \rho(y) d y, \\
d \rho(y) & =\frac{(1-y)^{\alpha}(1+y)^{\beta} d y}{\int_{-1}^{1}(1-x)^{\alpha}(1+x)^{\beta} d x} .
\end{aligned}
$$

For further discussion of the process we refer the reader to [18]. It is proved in [18] that (8.21) is $\mathrm{TP}_{\infty}$ in the variables $x$ and $y$. Since (8.21) for $\alpha=\beta=\gamma-1 / 2$ is a symmetric process we deduce by virtue of Theorem 6.5 that

$$
p(t ; 0, y)=\sum_{u=0}^{\infty} e^{-\lambda_{n} t} P_{n}(y) w_{n}
$$

is $\mathrm{TP}_{\infty}$ for $t>0$ and $0 \leqq y<1$.

C. We consider a general linear diffusion process whose infinitesimal operator is characterized by the generalized diffusion equation

$$
\frac{\partial p}{\partial t}=\frac{\partial}{\partial m} \frac{\partial p}{\partial x}=\mathscr{G} p, \quad a<x<b .
$$

This should be interpreted in the Feller sense [8] where $m$ is a $\sigma$-finite measure with the property that every open subset of $[a, b]$ has positive measure. For the full meaning of (8.23) and its significance we refer to [8], [5]. Itô and McKean 
(unpublished)(see also [28]) have constructed a strong Markoff process with continuous path functions under the condition that the transition density function satisfies certain classical boundary conditions at $a$ and $b$ or that the end points are exit or natural boundaries [28]. The associated resolvent equation

$$
(\lambda-\mathscr{G}) p=0
$$

subject to the same boundary conditions admits a Green's function of the form

$$
G(x, y, \lambda)= \begin{cases}u(x, \lambda) v(y, \lambda), & x \leqq y \\ u(y, \lambda) v(x, \lambda), & x>y\end{cases}
$$

where $u(x, \lambda)$ is positive, strictly increasing, satisfies (8.24) and obeys the proper boundary condition at $a$. Similarly $v(x, \lambda)$ is positive, strictly decreasing and obeys the appropriate boundary condition at $b$. We suppose for simplicity that $a$ and $b$ are accessible. Itô and McKean prove that $u(x, \lambda)=E\left(e^{-\lambda \sigma_{b}(x)}\right)$ where $\sigma_{b}(x)$ denotes the first-passage time from $x$ to $b$ and $E$ designates the operation of expected value. Similarly, $v(y, \lambda)=E\left(e^{-\lambda \sigma_{a}(y)}\right)$. By Theorem 5.1 the density function (which exists [28]) of $\sigma_{b}(x)$ is $\mathrm{R}_{\infty}$, and the density function of $\sigma_{a}(y)$ is $\mathrm{TP}_{\infty}$. In accordance with our comments about (7.4) we infer that

$$
\begin{aligned}
& u(x, \lambda) \text { is } \mathrm{TP}_{\infty} \text { for } a<x<b \text { and } \lambda>0, \\
& v(y, \lambda) \text { is } \mathbf{R R}_{\infty} \text { for } a<y<b \text { and } \lambda>0 .
\end{aligned}
$$

A similar result to (8.25) holds whenever the boundaries are exit or natural. In the regular case the same conclusion is valid for boundary conditions of classical type.

9. More examples (analytic methods). In $\S 8$, paragraph 9, it was pointed out that the Bessel function $I_{n}(t)$ possesses a variety of sign-regular properties. The method of analysis was based on probability considerations. In the present section we employ special analytical arguments and derive more extensive results for special cases. Consider the density function

$$
f(x, \alpha)= \begin{cases}e^{-x} \sum_{n=0}^{\infty} \frac{c_{n} x^{\alpha+n}}{\Gamma(\alpha+n+1)}, & x>0, \\ 0, & x \leqq 0 .\end{cases}
$$

We assume that $c_{n}>0$ and $\sum c_{n}=1$. The special case that arises for $c_{n}=e^{-\lambda \lambda^{n} ! n !}$ ( $\lambda$ a positive fixed parameter) and $\alpha$ a half integer is the familiar noncentral chi square density.

We start with two lemmas; the first exemplifies a smoothing device rather common to these kinds of studies.

LEMMA 9.1. Let $f(x)$ vanish for $x \leqq 0$ and satisfy the following conditions:

(i) $f(x+y)$ is $\mathrm{ERR}_{n}$ for $x, y>0$ (this hypothesis entails that $f(x)$ is $C^{2 n-2}$ for $0<x<\infty$ ). 
(ii) $\left|f^{(n-1)}(x)\right|\left[\right.$ and hence $\left|f^{v}(x)\right|$ for $\left.0 \leqq v<n-1\right]$ is integrable on any finite interval and is dominated by $C e^{a x}$ for $C>0$, some $a>0$.

(iii) $f^{(v)}(0)=0$ for $0 \leqq v \leqq n-2$.

Then $f(x-y)$ is $\operatorname{TP}_{n}$ for $-\infty<x, y<\infty$.

Proof. Hypothesis (i) implies by (1.5)

$$
(-1)^{v(v+1) / 2} f^{*}\left(\begin{array}{l}
u_{1}, u_{2}, \cdots, u_{v+1} \\
0,0, \cdots, 0
\end{array}\right)>0
$$

for $0<u_{1}<u_{2}<\cdots<u_{v+1} ; v \leqq n-1$, where (9.2) is calculated based on the function $f(x+y)$.

Let

$$
g_{\sigma}(x)=\frac{1}{\sqrt{ }(2 \pi) \sigma} \exp \left[-\frac{1}{2}\left(\frac{x}{\sigma}\right)^{2}\right] \text { for }-\infty<x<\infty ; \sigma>0 .
$$

We form the convolution

$$
h_{\sigma}(x)=\int_{0}^{\infty} g_{\sigma}(x-u) f(u) d u, \quad-\infty<x<\infty .
$$

It follows that

$$
h_{\sigma}^{(v)}(x)=\int_{0}^{\infty} g_{\sigma}^{(v)}(x-u) f(u) d u, \quad-\infty<x<\infty, v \geqq 0,
$$

and differentiatiation under the integral sign is justified by hypothesis (ii). Hypotheses (i) and (ii) also insure the existence of

$$
\int_{0}^{\infty} g_{\sigma}^{\left(v_{1}+1\right)}(x-u) f^{\left(v_{2}-1\right)}(u) d u
$$

for $v_{1} \geqq 0 ; 1 \leqq v_{2} \leqq n-1$.

Integrating by parts, which is permissible by virtue of hypothesis (ii), we obtain

$$
\begin{aligned}
\int_{0}^{\infty} g_{v}^{\left(v_{1}+1\right)}(x-u) f^{\left(v_{2}-1\right)}(u) d u= & \int_{0}^{\infty}-\frac{\partial}{\partial u} g_{\sigma}^{\left(v_{1}\right)}(x-u) f^{\left(v_{2}-1\right)}(u) d u \\
= & {\left[-g_{\sigma}^{\left(v_{1}\right)}(x-u) f^{\left(v_{2}-1\right)}(u)\right]_{0}^{\infty} } \\
& +\int_{0}^{\infty} g_{\sigma}^{\left(v_{1}\right)}(x-u) f^{\left(v_{2}\right)}(u) d u
\end{aligned}
$$

The boundary terms vanish for $1 \leqq v_{2} \leqq n-1$ by hypothesis (iii). Hence, by induction

$$
h_{\sigma}^{\left(v_{1}+v_{2}\right)}(x)=\int_{0}^{\infty} g_{\sigma}^{\left(v_{1}\right)}(x-u) f^{\left(v_{2}\right)}(u) d u
$$


for $-\infty<x<\infty ; v_{1} \geqq 0 ; 0<v_{2} \leqq n-1$. Applying the composition formula (0.4) we obtain

$$
\begin{gathered}
(-1)^{v(v+1) / 2}\left|h_{\sigma}^{(k+l)}(x)\right|_{k, l=0}^{v}=h_{\sigma}^{*}\left(\begin{array}{l}
x, x, \cdots, x \\
0,0, \cdots, 0
\end{array}\right) \\
=\int_{0<u_{1}<u_{2}<\ldots<u_{v+1}} g^{*}\left(\begin{array}{c}
x, x, \cdots, x \\
u_{1}, u_{2}, \cdots, u_{v+1}
\end{array}\right) f^{*}\left(\begin{array}{c}
u_{1}, u_{2}, \cdots, u_{v+1} \\
0,0, \cdots, 0
\end{array}\right) \\
\times d u_{1} d u_{2} \cdots d u_{v+1}
\end{gathered}
$$

the determinants are each of size $v+1 \times v+1(0 \leqq v \leqq n)$. (The $*$ determinants in (9.4) are based on $h_{\sigma}(x-y), g_{\sigma}(x-y)$, and $f(x-y)$, respectively.) Since $g_{\sigma}(x-y)$ is ETP of all orders we infer after referring to (9.2) that $h_{\sigma}(x-y)$ is $\mathrm{ETP}_{n+1}$ and so $\mathrm{TP}_{n+1}$. Taking the limit $\sigma \rightarrow 0+$, we conclude that $f$ is $\mathbf{T P}_{n+1}$.

The next lemma establishes the sign regularity of the function

$$
K(i, j)=\frac{1}{\Gamma(\alpha+i+j)}, \quad i, j=0,1,2, \cdots .
$$

Lemma 9.2. Let $a_{i j}=1 / \Gamma(\alpha+i+j)(=0$ if $\alpha+i+j$ is a nonpositive integer). Then for $\alpha>-v$,

$$
(-1)^{v(v+1) / 2} A\left(\begin{array}{l}
i_{0}, i_{1}, \cdots, i_{v} \\
j_{0}, j_{1}, \cdots, j_{v}
\end{array}\right)>0 \text { for }\left\{\begin{array}{l}
0 \leqq i_{0}<i_{1}<\cdots<i_{v} \\
0 \leqq j_{0}<j_{1}<\cdots<j_{v}
\end{array}\right.
$$

Proof. We assume inductively that (9.5) is already proved for all determinants of order $\leqq v$. The case $v=0$ is trivial. In order to advance the induction we consider first the case $i_{k}=j_{k}=k(0 \leqq k \leqq v)$. Factor out $a_{v j}$ from the $j$ th column $(0 \leqq j \leqq v)$. Then

and define

$$
\prod_{j=0}^{v} a_{v j}=\prod_{j=0}^{v} \frac{1}{\Gamma(\alpha+v+j)}=\frac{\prod_{k=0}^{v-1}(\alpha+v+k)^{k+1}}{[\Gamma(\alpha+2 v)]^{v+1}}
$$

$$
C_{i j}=\frac{a_{i j}}{a_{v j}}= \begin{cases}\frac{\Gamma(\alpha+v+j)}{\Gamma(\alpha+i+j)}=\prod_{k=i}^{v-1}(\alpha+k+j) & \text { for } i<v \\ 1 & \text { for } i=v\end{cases}
$$

Then

$$
A\left(\begin{array}{l}
0,1, \cdots, v \\
0,1, \cdots, v
\end{array}\right)=\frac{\prod_{k=0}^{v-1}(\alpha+v+k)^{k+1}}{[\Gamma(\alpha+2 v)]^{v+1}}|C(\alpha, v)|
$$

where $C(\alpha, v)$ denotes the $v+1$ order matrix $\left\|C_{i j}\right\|_{i, j=0}^{v}$. Let $C_{j}(\alpha, v)$ be the jth column of $C(\alpha, v)$ 


$$
C_{j}^{\prime}(\alpha, v)= \begin{cases}C_{j}(\alpha, v)-C_{j-1}(\alpha, v) & \text { for } j>0 \\ C_{0}(\alpha, v) & \text { for } j=0 .\end{cases}
$$

Then, for $i<v, j>0$.

$$
\begin{aligned}
C_{i j}^{\prime} & =C_{i j}-C_{i, j-1}=\prod_{k=i}^{v-1}(\alpha+k+j)-\prod_{k=i}^{v-1}(\alpha+k+j-1) \\
& =(v-i) \prod_{k=i}^{v-2}(\alpha+k+j) \quad \text { if } i<v-1 ; 1 \text { if } i=v-1 .
\end{aligned}
$$

While for $i=v$

$$
C_{v j}^{\prime}=\left\{\begin{array}{lll}
0 & \text { for } & j>0 \\
1 & \text { for } & j=0
\end{array}\right.
$$

Let $D$ be the matrix obtained from $C^{\prime}$ by deleting the last row first column. Then $|C(\alpha, v)|=(-1)^{v}|D|$. But $D=v ! C(\alpha, v-1)$. Hence, by iteration $|C(\alpha, v)|=(-1)^{(v+1) v / 2} \prod_{k=1}^{v} k$ ! since $|C(\alpha, 0)|=1$. Thus

$$
(-1)^{v(v+1) / 2} A\left(\begin{array}{l}
0,1, \cdots, v \\
0,1, \cdots, v
\end{array}\right)=\frac{\prod_{k=0}^{v-1}(\alpha+v+k)^{k+1}}{[\Gamma(\alpha+2 v)]^{v+1}} \prod_{k=1}^{v} k !>0 \text { since } \alpha>-v .
$$

Further

$$
(-1)^{v(v+1) / 2} A\left(\begin{array}{c}
m, m+1, \cdots, m+v \\
n, n+1, \cdots, n+v
\end{array}\right)>0 \text { for } m, n>0
$$

since we can reduce it to the previous case by setting $\alpha^{\prime}=\alpha+m+n$. Applying Fekete's Theorem [ $\$ 9$, Chapter 5] the lemma follows.

Another method of proof consists of applying the composition formula (0.4) on the representation

$$
\frac{1}{\Gamma(\alpha+k+l)}=\frac{1}{\Gamma(k+\alpha / 2) \Gamma(l+\alpha / 2)} \int_{0}^{1} t^{k+\alpha / 2-1}(1-t)^{l+\alpha / 2} d t
$$

and using the fact that a Vandermonde type determinant $\left|\left[u\left(t_{j}\right)\right]^{n_{i}}\right|$ is positive whenever $t_{0}<t_{1}<\cdots<t_{v}$ and $0 \leqq n_{0}<n_{1}<\cdots<n_{v}$ where $u(t)$ is a strictly increasing function of $t$. This argument can only be justified for $\alpha>0$ since the above integral is not meaningful for negative $\alpha$ and arbitrary integers $k$ and $l$.

We now turn to the first theorem of this section.

THEOREM 9.1. Let $\left\langle C_{n}\right\rangle_{-\infty}^{\infty}$ be real constants satisfying

(i) $C_{n}=0$ for $n<0$.

(ii) $C_{n-m}$ is $\mathrm{TP}_{r}$ for $-\infty<n, m<\infty$.

(iii) $0<\sum_{n=0}^{\infty} C_{n}<\infty$. 


$$
f_{\alpha}(x)=\left\{\begin{array}{lc}
\sum_{n=0}^{\infty} C_{n} \frac{x^{\alpha+n}}{\Gamma(\alpha+n+1)} & \text { for } x>0, \\
0 & \text { for } x \leqq 0 .
\end{array}\right.
$$

Then $f_{\alpha}(x-y)$ is $\operatorname{TP}_{r}$ for $-\infty<x, y<\infty$.

Proof. Appealing to Lemma 9.1, the theorem will follow if we verify that $f_{a}(x+y)$ is ERR, since the other conditions of Lemma 9.1 are clearly satisfied Our task is to prove that, for $A=\left\|f_{\alpha}^{(i+j)}(x)\right\|_{i, j=0}^{v}(0 \leqq v \leqq r-1)$ and $0<x<\infty$ ( $x$ fixed), then $(-1)^{v(v+1) / 2}|A|>0$. Let

$$
d_{i}=f_{\alpha}^{(2 v-i)}(x)=\sum_{n=0}^{\infty} C_{n} \frac{x^{\alpha+n-2 v+i}}{\Gamma(\alpha+n-2 v+i+1)}, \quad 0 \leqq i \leqq 2 v ; 0 \leqq v \leqq r-1 .
$$

Let $D=\left\|d_{i+j}\right\|=\left\|f_{\alpha}^{(2 v-i-j)}(x)\right\|_{i, j=0}^{n}$. Then obviously $|D|=|A|$. Consider

$$
d_{i+j}=\sum_{n=0}^{\infty} C_{n} \frac{x^{\alpha+n-2 v+i+j}}{\Gamma(\alpha+n-2 v+i+j+1)} .
$$

The change of variable $m=n+i$ yields

$$
\begin{aligned}
d_{i+j} & =x^{\alpha-2 v} \sum_{m=i}^{\infty} C_{m-i} \frac{x^{m+j}}{\Gamma(\alpha+m-2 v+j+1)} \\
& =x^{\alpha-2 v} \sum_{m=0}^{\infty} C_{m-i} \frac{x^{m+i}}{\Gamma(\alpha+m-2 v+j+1)} \text { by hypothesis (i). }
\end{aligned}
$$

Let $C(i, m)=c_{m-i}$ and $R(m, j)=x^{m+j} / \Gamma(\alpha+m-2 v+j+1)$. Let $\beta=\alpha+1-2 v$. Then, since $\alpha>r-2$ and $v-1 \leqq r-2, \beta=\alpha-(v-1)-v>-v$. Hence, by Lemma $9.2,1 / \Gamma(\alpha+m-2 v+j+1)$ is $\mathbf{R R}_{r}$ for $m, j \geqq 0$. Also, $R(m, j)$ is $\mathbf{R R}_{r}$ for $m, j \geqq 0$, since the powers of $x$ factor in evaluating the relevant determinants. We can write (9.7) in the form

$$
d_{i+j}=x^{\alpha-2 v} \sum_{m=0}^{\infty} C(i, m) R(m, j) .
$$

Applying the composition formula (0.4), we obtain

$$
|D|=x^{\gamma} \underset{0 \leqq m_{0}<m_{1}<\ldots<m_{v}}{\sum \ldots \sum} C\left(\begin{array}{l}
0,1, \cdots, v \\
m_{0}, m_{1}, \cdots, m_{v}
\end{array}\right) R\left(\begin{array}{l}
m_{0}, m_{1}, \cdots, m_{v} \\
0,1, \cdots, v
\end{array}\right)
$$

where $\gamma$ is a suitable real number. By condition (ii) we know that the first factor of the sum is constantly non-negative and if $c_{l_{0}}>0, c_{l}=0\left(l<l_{0}\right)$ then

$$
C\left(\begin{array}{l}
0,1, \quad \cdots, \quad v \\
l_{0}, l_{0}+1, \cdots, l_{0}+v
\end{array}\right)>0 .
$$

According to Lemma 9.2, 


$$
(-1)^{v(v+1) / 2} R\left(\begin{array}{l}
m_{0}, m_{1}, \cdots, m_{v} \\
0,1, \cdots, v
\end{array}\right)>0 ;
$$

therefore $(-1)^{v(v+1) / 2}|D|>0$ as desired to be shown.

COROLlary 9.1. Let $f_{\alpha}$ satisfy the conditions of Theorem 9.1 and define $k_{\alpha}(x)=e^{-x} f_{\alpha}(x)(-\infty<x<\infty)$; then $k_{\alpha}(x-y)$ is $\mathrm{TP}_{r}$.

Proof. The result is immediate by virtue of the identity

$$
k_{\alpha}\left(\begin{array}{l}
x_{1}, x_{2}, \cdots, x_{p} \\
y_{1}, y_{2}, \cdots, y_{p}
\end{array}\right)=\exp \left(-\sum_{i=1}^{p} x_{i}\right) \exp \left(\sum_{i=1}^{p} y_{i}\right) f_{\alpha}\left(\begin{array}{c}
x_{1}, x_{2}, \cdots, x_{p} \\
y_{1}, y_{2}, \cdots, y_{\gamma}
\end{array}\right) .
$$

An important example of Corollary 9.1 is

$$
k_{\alpha}(x ; \lambda)= \begin{cases}e^{-x} e^{-\lambda} \sum_{n=0}^{\infty} \frac{\lambda^{n}}{n !} \frac{x^{n+\alpha}}{\Gamma(n+\alpha+1)}, & x \geqq 0, \\ 0, & x<0,\end{cases}
$$

where $k_{\alpha}(x-y ; \lambda)$ is $\mathrm{TP}_{r}$ for $\alpha>r-2$. The assumption $\alpha>r-2$ is essential. In fact, consider the example

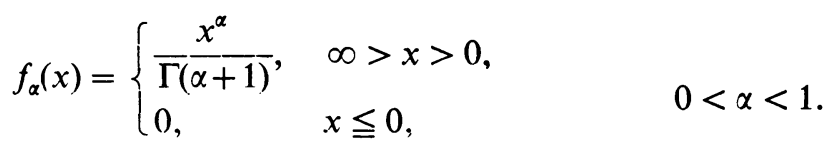

A trivial explicit calculation shows that $f_{\alpha}\left(\begin{array}{l}3,4,5 \\ 1,2,3\end{array}\right)<0$. However, in the case that $\alpha=$ non-negative integer we can obtain a sharper theorem.

Lemma 9.3. Suppose $\left\{c_{n}\right\}$ satisfy the conditions of Theorem 9.1. Let $\alpha$ be a non-negative integer and define $f_{\alpha}(x)$ according to (9.6). Then either $f_{\alpha}(x)$ is an exponential polynomial of order $k \leqq r-1$ or $f_{\alpha}(x+y)$ is $\mathrm{ER}_{\boldsymbol{r}}$ for $0<x, y<\infty$. (An exponential polynomial of order $k$ is an expression of the form

$$
y=P_{1}(x) e^{\alpha_{1} x}+\cdots+P_{s}(x) e^{\alpha_{s} x} \quad\left(\alpha_{i} \neq \alpha_{j} \text { if } i \neq j\right)
$$

where $P_{1}, \cdots, P_{s}$ are polynomials with $\operatorname{deg} P_{i}=k_{i}-1$ and $\sum_{i=1}^{s} k_{i}=k$.)

Proof. We note that when $\alpha$ is a non-negative integer, $f_{\alpha}(x)$ is an entire function for which

$$
f_{\alpha}^{\left(v_{1}+v_{2}\right)}(0)=c_{v_{1}+v_{2}-\alpha} .
$$

The $(v+1)$ st order $(v+1 \leqq r)$ determinant

$$
T_{\alpha, v}(x)=f_{\alpha}^{*}\left(\begin{array}{l}
x, x, \cdots, x \\
0,0, \cdots, 0
\end{array}\right)
$$

based on $f_{\alpha}(x+y)$ is also obviously an entire function whose coefficients in its power series expansion are $T_{\alpha, v}^{(p)}(0) ! p !(p=0,1,2, \cdots)$. Differentiating (9.11), we find that $T_{a, v}^{(p)}(0) / p$ ! is a sum of determinants of the form 


$$
C\left(\begin{array}{ccc}
0,1, \cdots, v-p, v-p+1+s_{1}, v-p+2+s_{2}, \cdots, v+s_{p} \\
-\alpha,-\alpha+1, \cdots, & \cdots & , \cdots,-\alpha+v
\end{array}\right)
$$

the rows and columns as indicated; the indices appear in natural increasing order, $s_{j} \geqq 0(j=1, \cdots, p)$ and $\sum_{j=1}^{p} s_{j}=p$. Since $c_{n-m}$ is $\mathrm{TP}_{r}$ by hypothesis, it follows reversing the order of columns that each of these terms has the sign $(-1)^{v(v+1) / 2}$. Thus we have established the representation

$$
(-1)^{v(v+1) / 2} T_{\alpha, v}(x)=\sum_{p=0}^{\infty} b_{p} x^{p}, \quad 0<x<\infty,
$$

where $b_{n} \geqq 0$. Therefore it is manifestly evident that either (9.11) is identically zero, in which case $f_{\alpha}(x)$ is an exponential polynomial of order at most $v-1 \leqq r-1$ [36] or

$$
(-1)^{v(v+1) / 2} T_{\alpha, v}>0, \quad 0<x<\infty .
$$

Of course, if (9.13) holds for a certain $v_{0}$ then strict inequality in (9.13) holds for all $v \leqq v_{0}$.

If the case (9.13) holds for $v=0,1, \cdots, r-1$ then we obtain that $f_{\alpha}(x+y)$ is $\mathrm{ERR}_{r}$ in $x, y \geqq 0$ as was to be shown. This completes the proof of the lemma.

We remark that if in Lemma 9.3 we had assumed that $c_{n+m}$ was $\operatorname{TP}_{r}$ then by [36] the statement of the lemma would have been that either

$$
f_{\alpha}(x)=\sum_{i=1}^{k} a_{i} e^{\gamma_{i} x}, \quad a_{i}>0(i=1, \cdots, k \leqq r-1),
$$

where the $\gamma_{i}$ are real and distinct (in which case necessarily $\alpha=0$ ) or $f_{\alpha}(x+y)$ is $\mathrm{ETP}_{r}$ for $0<x, y<\infty$.

We point out some formula concerning the sign-regular properties of the function (9.9) with respect to the variables $(x, \lambda)$. An easy application of the composition formula (0.4) implies that $f_{\alpha}(x, \lambda)$ is $\mathrm{ERR}_{\infty}$ for $x>0$ and $\lambda>0$.

Finally we consider the function (9.6) $f_{\alpha+\beta}(x)=U(\alpha+\beta)$ with $x>0$ fixed and where $\alpha$ and $\beta$ traverse the set of non-negative integers. We impose the conditions of Theorem 9.1. The method of Theorem 9.1 applies, indeed, in a simpler fashion and we deduce that $f_{\alpha+\beta}(x)$ is $\operatorname{RR}_{\infty}$ for $\alpha, \beta,=0,1,2, \cdots$. We omit the details.

We next make some observations pertaining to the total positivity character of certain stable laws. We limit our discussion to the stable laws whose Laplace transformation is of the form

$$
\frac{1}{t^{1 / \alpha}} \int_{0}^{\infty} f\left(\frac{u}{t^{1 / \alpha}}\right) e^{-u s} d u=e^{-t s^{\alpha}}, \quad s>0(0<\alpha<1) .
$$

We recognized the known fact in writing (9.14), that these stable laws are distributions of non-negative random variables. 
Let $\tilde{F}_{\alpha}(z)$ denote the Mellin transform of $f_{\alpha}(u)$. A simple calculation starting with (9.14), $t=1$, yields

$$
\tilde{F}_{x}(1-z)=\frac{\frac{1}{\alpha} \Gamma\left(\frac{z}{\alpha}\right)}{\Gamma(z)} .
$$

We now specialize to the case $\alpha=1 / k, k=2,3,4, \cdots$. The duplication formula

$$
\Gamma(k z)=\frac{\Gamma(z) \Gamma\left(z+\frac{1}{k}\right) \cdots \Gamma\left(z+\frac{k-1}{k}\right)}{(\sqrt{ }(2 \pi))^{k-1} k^{1 / 2-k z}}
$$

inserted into (9.15) gives the formula

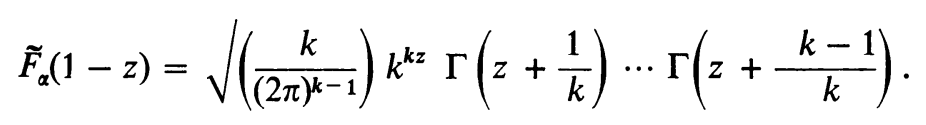

To invert the right-hand side of (9.17) we use the following familiar relations for Mellin transforms. Throughout, a capital letter with a curl sign represents the Mellin transform of the corresponding function.

(a) If $\tilde{G}(z) \sim g(u), \tilde{H}(z) \sim h(u)$, then $\tilde{G}(x) \tilde{H}(z) \sim \int_{0}^{\infty} f(u / v) h(v)(d v / v)$.

(b) If $\tilde{G}(z) \sim g(u)$, then $\tilde{G}(1-z) \sim(1 / u) g(1 / u)$.

(c) If $\tilde{G}(z) \sim g(u)$, then $a^{2} \tilde{G}(z) \sim g(u / a), a>0$.

(d) $\Gamma(z+c) \sim e^{-u} u^{c}$ and hence $\Gamma(1-z+c) \sim u^{-r-1} e^{-1 / u}$.

It follows that $f_{1 / k}(u)$ can be represented as a $k-2$ fold integral whose integrand involves products of exponentials and powers. In particular

and thus

$$
f_{1 / 3}(u)=\frac{1}{2 \pi \sqrt{ } 3} u^{-4 / 3} \int_{0}^{\infty} e^{-v / 27 u} e^{-1 / v} v^{-4 / 3} d v
$$

$$
\frac{1}{t^{3}} f_{1 / 3}\left(\frac{u}{t^{3}}\right)=\frac{1}{2 \pi \sqrt{ } 3} t u^{-4 / 3} \int_{0}^{\infty} e^{-v t^{3 / 27 u}} e^{-1 / v} v^{-4 / 3} d v
$$

$$
=\frac{1}{2 \pi \sqrt{ } 3} t^{2} u^{-4 / 3} \int_{0}^{\infty} e^{-\xi / 27 u} e^{-t^{3} / \xi} \xi^{-4 / 3} d \xi .
$$

The function $e^{-\xi / 27 u}$ is clearly $\operatorname{STP}_{\infty}$ for $\xi>0$ and $u>0$. Similarly $\exp \left[-t^{1 / 3} / \xi\right]$ is $\operatorname{STP}_{\infty}$ for $\xi>0$ and $t>0$. Applying (0.4) we infer that $f_{1 / 3}\left(u / t^{3}\right)$ is $\operatorname{STP}_{\infty}$ for $u>0$ and $t>0$.

The inversion process with the aid of properties (a)-(d) also yields

$$
\frac{1}{t^{4}} f_{1 / 4}\left(\frac{u}{t^{4}}\right)=\frac{2^{-5}}{(2 \pi)^{3 / 2}} u^{-7 / 4} t^{2} \int_{0}^{\infty} \eta^{-1 / 2} \exp \left[-\frac{\eta}{4^{4} u}\right]
$$

$$
\cdot\left(\int_{0}^{\infty} e^{-\xi / \eta} e^{-t^{4} / \xi} \xi^{-5 / 4} d \xi\right) d \eta .
$$


A double application of $(0.4)$ shows that $f_{1 / 4}\left(u / t^{4}\right)$ is $\operatorname{STP}_{\infty}$ for $u>0$ and $t>0$. By these same methods we deduce generally that

$$
f_{1 / k}\left(\frac{u}{t^{k}}\right) \text { is } \operatorname{STP}_{\infty} \text { for } u>0 \text { and } t>0 .
$$

The proof is accomplished by induction. Let

$$
\Gamma\left(1-z+\frac{2}{k}\right) \Gamma\left(1-z+\frac{3}{k}\right) \cdots \Gamma\left(1-z+\frac{k-1}{k}\right) \sim g(u) .
$$

Suppose we have proved that $g\left(u / t^{k}\right)$ is $\operatorname{STP}_{\infty}$ for $u>0$ and $t>0$. Then by (a), (d) and (c) we have

$$
\begin{aligned}
f_{1 / k}\left(\frac{u}{t^{k}}\right) & =C_{k} u^{-(k+1) / k} t^{k+1} \int e^{-v t^{k} / a_{k} u} v^{1 / k} g(v) d v \\
& =C_{k} u^{-(k+1) / k} \int e^{-v / a_{k} u} v^{1 / k} g\left(\frac{v}{t^{k}}\right) d v
\end{aligned}
$$

where $a_{k}=k^{k}$ and $C_{k}$ is a suitable normalizing constant. Now applying $(0.4)$ the result follows.

Expansion of $g(v)$ in (9.21) furnishes a representation as a $k-2$ fold multiple integral.

Returning now to the case of general rational $\alpha=m / n(m<n)$. Using the duplication formula twice in $(9.15)$ gives

$$
F_{m / n}(1-z)=\frac{\sqrt{ }\left(\frac{n}{m}\right) n^{n z} \Gamma\left(\frac{z}{m}+\frac{1}{n}\right) \Gamma\left(\frac{z}{m}+\frac{2}{n}\right) \cdots \Gamma\left(\frac{z}{m}+\frac{n-1}{n}\right)}{(\sqrt{ }(2 \pi))^{n-m} m^{m z} \Gamma\left(\frac{z}{m}+\frac{1}{m}\right) \Gamma\left(\frac{z}{m}+\frac{2}{m}\right) \cdots \Gamma\left(\frac{z}{m}+\frac{m-1}{m}\right)}
$$

In order to invert (9.22) in simple terms, we need properties (a)-(d) and the further relations

$$
\frac{\Gamma(z)}{\Gamma(z+d)} \sim g(u)=\left\{\begin{array}{ll}
\frac{1}{\Gamma(d)}(1-u)^{d-1} & \text { for } 0<u<1, \\
0, & \text { otherwise, }
\end{array} \quad(d>0) .\right.
$$

(f) If $\tilde{G}(z) \sim g(u)$, then $\tilde{G}(z / \alpha) \sim \alpha g\left(u^{\alpha}\right)$ for $\alpha>0$.

Again we achieve a representation of $f_{m / n}(u)$ as an $n-2$ fold integral. For example,

$$
f_{2 / 3}(u)=C u^{-7 / 3} \int_{0}^{1} \exp \left(-\frac{2^{4} 3^{-6}}{\xi^{2} u^{2}}\right) \xi^{-5 / 3}\left(1-\xi^{2}\right)^{-5 / 6} d \xi
$$

where $C$ is an appropriate constant. It is an open problem whether $f_{2 / 3}\left(u / t^{3 / 2}\right)$ is still $\mathrm{TP}_{\infty}$. 
Combining Theorem 7.1 with the fact that $f_{1 / k}\left(u / t^{k}\right.$ is $\operatorname{TP}_{\infty}$ as established we note that any symmetric stable law of order $\alpha=2 / k(k=2,3,4, \cdots)$ with density $g_{\alpha}\left(u / t^{1 / \alpha}\right)$ where

$$
\int_{-\infty}^{\infty} e^{i z u} g(u) d u=e^{-|z|^{\alpha}}
$$

is $\mathrm{TP}_{\infty}$ for $u>0$ and $t>0$. This follows from the fact that Brownian motion subordinated in the sense of Theorem 7.1 with the positive stable process of order $1 / k$ produces the symmetric stable process of order $2 / k$. Invoking Theorem 7.1 , part (ii), gives the desired conclusion.

10. Unimodality, ratio theorems and rates of ergodicity. A function totally positive or more generally sign regular is endowed with certain structural properties pertaining to monotonicity, unimodality, rate of growth at infinity, etc. The developments of this section center on these properties, with emphasis on their interpretations and implications for the probability functions introduced in $\S 2$.

A series of propositions are stated and proved. We do not attempt to exhaust the full range of possibilities; we have emphasized mostly those possessing probabilistic interest.

We start with the concept of unimodality.

Proposition 10.1. If $K(u)$ is $\mathrm{PF}_{2}$, then $K(u)$ is unimodal.

In order to avoid technical details of no essential relevance the proof is carried out assuming $K(u)>0$ and $K^{\prime}(u)$ exists everywhere (see [33]). Then according to $(1.2)$, we have

$$
K^{*}\left(\begin{array}{l}
x_{1}, x_{2} \\
y, y
\end{array}\right)=\left|\begin{array}{ll}
K\left(x_{1}-y\right) & -K^{\prime}\left(x_{1}-y\right) \\
K\left(x_{2}-y\right) & -K^{\prime}\left(x_{2}-y\right)
\end{array}\right| \geqq 0
$$

for $x_{1}<x_{2}$ and $y$ arbitrary. In the event that $K^{\prime}\left(u_{0}\right)=0$ the inequality implies that $K^{\prime}(u) \geqq 0$ for $u<u_{0}$ and $K^{\prime}(u) \leqq 0$ for $u>u_{0}$. This clearly implies that $K(u)$ is unimodal. The same argument proves

Proposition 10.2. If $K(x+y)$ is $\mathrm{TP}_{2}(x, y \geqq 0)$ then $K(u)$ has a single minimum (i.e., there does not exist points $x>y>z \geqq 0$ such that $K(x)<K(y)$ and $K(z)$ $<K(y)$ ). In addition, if $K(u) \rightarrow 0$ as $u \rightarrow \infty$ then $K(u)$ is decreasing for $u \geqq 0$.

Propositions 10.1 and 10.2 deal with the case where the argument of $K(u)$ is a continuous variable. We note without proof that the corresponding result is valid in case $K_{n}$ is a $\mathrm{PF}_{2}$ sequence or if $K_{n+m}$ is $\mathrm{TP}_{2}$ for $n \geqq 0, m \geqq 1$.

Taking advantage of Propositions 10.1 and 10.2 and the foregoing remark we 
now deduce the property of unimodality for several of the probability functions introduced in the previous sections. The complete discussion is offered in the Markoff chain case. At the close of this section the analogous results are stated for the case of a general Markoff process.

Let $\mathscr{P}$ denote a temporally homogeneous Markoff chain satisfying the conditions of Theorem 2.6 with $r \geqq 2$. This hypothesis will remain in force for Propositions 3-7 without further repetition.

The sample paths of $\mathscr{P}$ are depicted, as usual, by the random variables $X(n), n=0,1, \cdots$. Let $P_{i j}$ denote the probability transition matrix of the process.

Proposition 10.3. $P_{00}^{n}$ is decreasing in $n(n \geqq 0)$.

Since $P_{0 j}^{n}$ is $\mathrm{TP}_{2}$ in $n, j \geqq 0$ the result follows using the relation $P_{00}^{n}=\sum_{j=0}^{\infty} P_{0 j}^{n} \delta_{j 0}$ and the fact that $P_{0 j}^{n}$ carries decreasing sequences into decreasing sequences (see $\$ 6$, II).

Let $F_{00}^{n}=$ the probability that the first return to state zero occurs at time $n, n \geqq 0\left(F_{00}^{0}=0\right.$ by convention). We shall prove that $F_{00}^{n+m}$ is $\mathrm{TP}_{r}$ for $n \geqq 0$, $m \geqq 1$ and hence that $F_{00}^{m}, m \geqq 1$, is decreasing by Proposition 10.2.

To this end, we first introduce the probability quantities

$$
{ }_{k} P_{i j}^{n}=\operatorname{Pr}\{X(n)=j, X(v) \neq k, 1 \leqq v<n \mid X(0)=i\}, \quad n \geqq 1 .
$$

In other words, ${ }_{k} P_{i j}^{n}$ denotes the transition probability function under the condition that the state $k$ is not entered in the intervening time. The concept of transition probabilities involving taboo states appears in the work of Harris (see also Chung [3]).

We may adapt the methods of Theorem 2.6 and prove that ${ }_{0} P_{0 j}^{n}$ is $\mathrm{TP}_{r}$ for $n \geqq 0$ and $j \geqq 0\left({ }_{0} P_{0 j}^{0}=\delta_{0 j}\right)$. The proof is based on the identity

$$
{ }_{0} P_{0 j}^{n}=\sum_{k=1}^{\infty}{ }_{0} P_{0 k}^{n-1} P_{k j}, \quad n \geqq 2, \quad j \geqq 0 .
$$

Now

$$
F_{00}^{n+m}=\sum_{k=1}^{\infty}{ }_{0} P_{0 k}^{n} F_{k 0}^{m}, \quad n \geqq 0, \quad m \geqq 1 .
$$

By what was just proved we know that ${ }_{0} P_{0 k}^{n}$ is $\mathrm{TP}_{r}$ for $n \geqq 0$ and $k \geqq 0$. Theorem 2.1, part (ii), tells us that $F_{k 0}^{m}$ is $\mathrm{TP}_{r}$ for $k \geqq 0, m \geqq 1$. Applying (0.4) we deduce that $F_{00}^{n+m}$ is $\operatorname{TP}_{r}(n \geqq 0, m \geqq 0)$ as claimed. In summary

PROPOSITION 10.4. $F_{00}^{n+m}$ is $\mathrm{TP}_{r}$ for $n \geqq 0, m \geqq 1$ and $F_{00}^{n}$ is decreasing for $n \geqq 1$.

Proposition 10.5. $F_{10}^{n+m}$ is $\mathrm{TP}_{r}$ for $n \geqq 0, m \geqq 0$.

To prove this we first note that ${ }_{0} P_{1 k}^{n}$ is $\operatorname{TP}_{r}$ for $n \geqq 0, k \geqq 1$. (Here ${ }_{0} P_{1 k}^{0}=\delta_{1 k}$.) 
This is a consequence of Theorem 2.6. In fact, the states of the process may be identified with the set $\{1,2,3, \cdots\}$ since state 0 is taboo. Now

$$
F_{10}^{n+m}=\sum_{k=1}^{\infty}{ }_{0} P_{1 k}^{n} F_{k 0}^{m}, \quad n \geqq 0, \quad m \geqq 1 .
$$

The desired conclusion now follows by applying (0.4) to (10.3) with reference to Theorem 2.1.

As a corollary we have that $F_{10}^{n}$ is decreasing for $n \geqq 1$.

Proposition 10.6. Let the process $\mathscr{P}$ be $\mathrm{TP}_{3}$. For fixed $j \geqq 0, P_{0 j}^{n}$ is unimodal in $n \geqq 0$.

Proof. We know by Theorem 2.6 that $P_{0 j}^{n}$ is $\mathrm{TP}_{3}$ for $j \geqq 0$ and $n \geqq 0$. This implies that the matrix $M_{j n}=P_{0 j}^{n}(n, j=0,1,2, \cdots)$ induces a variation diminishing transformation. Furthermore $\sum_{j=0}^{\infty} M_{j n}=1$ for all $n \geqq 0$. It follows that the relationship $d_{n}=\sum_{j=0}^{\infty} c_{j} M_{j n}$ and therefore $d_{n}-\kappa=\sum_{j=0}^{\infty}\left(c_{j}-\kappa\right) M_{j n}$ for any constant $\kappa$ entails $V\left[\left(d_{n}-\kappa\right)_{n=0}^{\infty}\right] \leqq V\left[\left(c_{j}-\kappa\right)_{j=0}^{\infty}\right]$ where $V$ denotes, as previously the number of sign changes of the indicated sequence. Moreover, if equality holds then the sequences $\left(d_{1}-\kappa\right)$ and $\left(c_{j}-\kappa\right)$ have the same ordering of sign changes. (See $\S 1$.) We choose specially $c_{j}=\delta_{j j_{0}}$, the Kronecker delta function. Then $\left\{c_{j}-\kappa\right\}$ for each constant $\kappa$ has at most two sign changes and consequently the same holds for $d_{n}-\kappa=P_{0 j_{0}}^{n}-\kappa$. This easily implies that $P_{0 j_{0}}^{n}$ is unimodal and the proof is finished.

Let ${ }_{j_{0}} Q_{0 k}^{n}=\operatorname{Pr}\left[X(n)=k, 0 \leqq X(v) \leqq j_{0}, v=1, \cdots, n \mid X(0)=0\right\}, n \geqq 1,{ }_{j} Q_{0 k}^{0}=\delta_{v k}^{0}$. The same type of reasoning as employed in Theorem 2.6 based on the identity

$$
j_{0} Q_{0 k}^{n}=\sum_{r=0}^{j_{0}}{ }_{j_{n}} Q_{0 r}^{n-1} P_{r k}, \quad n \geqq 2, \quad 0 \leqq k \leqq j_{0},
$$

leads to the conclusion that ${ }_{j_{0}} Q_{0 k}^{n}$ is $\mathrm{TP}_{r}$ for $n \geqq 0$ and $0 \leqq k \leqq j_{0}$.

Let $F_{0,[j o, \infty)}^{n}$ denote the probability that first passage into the set of states $\left[j_{0}+1, \infty\right)$ occurs at the $n$th transition under the starting condition, $X(0)=0$. We prove that $F_{0,\left[j_{0}+\infty\right)}^{n+m}$ is $\mathrm{RR}_{r}$ for $n \geqq 0, m \geqq 1$. Its verification uses the renewal relation

$$
F_{0 .[j o, \infty)}^{n+m}=\sum_{k=0}^{j_{0}}{ }_{j_{0}} Q_{0 k}^{n} F_{k .\left[j_{0}, \infty\right)}^{m}, \quad n \geqq 0, \quad m \geqq 1 .
$$

We know by Theorem 2.1 that $F_{k,\left[j_{0}, \infty\right)}^{m}$ is $S R R_{r}$ for $m \geqq 1$ and $0 \leqq k \leqq j_{0}$. Also, as noted above, ${ }_{j} Q_{0 k}^{n}$ is $\mathrm{TP}$, for $n \geqq 0$ and $0 \leqq k \leqq j_{0}$. By using (0.4) applied to (10.4) we obtain the following proposition.

Proposition 10.7. $F_{0,[j 0, \infty)}^{n+m}$ is $\mathrm{RR}$. for $n \geqq 0, m \geqq \Phi$.

As a corollary we may deduce from the $\mathrm{RR}_{2}$ property that $F_{0,[j o, \infty)}^{n}$ is decreasing for $n \geqq n_{0}$, where $n_{0}$ is the smallest $n$ for which $F_{0,\left[j_{0}, \infty\right)}^{n}$ is positive. 
By the identical techniques we may demonstrate that ${ }_{[i+1, \infty]} P_{i k}^{n}$ is $\operatorname{RR}_{r}$ for $n \geqq 0$ and $k \leqq i$ where

$$
[i+1, \infty) P_{i k}^{n}=\operatorname{Pr}\{X(n)=k, \quad X(v) \leqq i, v=1, \cdots, n \mid X(0)=i\}
$$

and ${ }_{[i+1, \infty)} P_{i k}^{0}=\delta_{i k}$. This result combined with Theorem 2.6 yields that the first-passage probability into the set $[i+1, \infty)$ whose initial state is $i, F_{i,[i+1, \infty)}^{n+m}$, is $\mathrm{TP}_{r}$ for $n \geqq 0, m \geqq 1$. In consequence, $F_{i,[i+1, \infty)}^{n}$ is decreasing for $n \geqq 1$.

We have directed attention so far to the property of unimodality for certain probability functions in which the time parameter serves as the variable. We now point out examples of unimodality for various probability functions of the state variable.

Proposition 10.8. (i) Suppose $\mathscr{P}$ is $\mathrm{TP}_{3}$ then $P_{i j o}$ is unimodal in $i$.

(ii) Let $F_{i 0}^{n}$ denote the probability that the first passage from state $i$ to state 0 occurs at time $n$. Suppose all states communicate and recurrence is certain, $i$ e., $\sum_{n=1}^{\infty} F_{i 0}^{n}=1$ for every $i$. Then $F_{i 0}^{n}$ for $n$ fixed is unimodal in $i$.

The proof in both cases paraphrases the arguments conducted for Proposition 10.6 of this section.

Strong ratio theorem. There is considerable literature concerned with the existence of $\lim _{n \rightarrow \infty}\left(P_{i j}^{n} / P_{k l}^{n}\right)$. Of course, to ensure that this ratio makes sense for $n$ sufficiently large, we postulate henceforth that the chain is aperiodic and that all states communicate. The classical Doeblin ratio theorem asserts that for any Markoff chain

$$
\lim _{n \rightarrow \infty} \frac{\sum_{m=1}^{n} P_{i j}^{m}}{\sum_{m=1}^{n} P_{k l}^{m}}
$$

exists, is finite and positive. The question of determining conditions which ensure that $\lim _{n \rightarrow \infty}\left(P_{i j}^{n} / P_{k l}^{n}\right)$ exists is considerably more delicate. (This property is commonly called the strong ratio theorem in comparison to the Doeblin ratio theorem.) An affirmative answer appears in the case of positive recurrent aperiodic Markoff chain in which all states communicate; its proof is trivial. There are known counterexamples to the strong ratio theorem in the general null recurrent Markoff chain [3]. On the other hand, the strong ratio theorem has been validated in the case of random walks [19] and for the case of spatially and temporally homogeneous Markoff processes [35]. We now show how the preceding theory can be used to prove a strong ratio theorem for another class of processes.

THEOREM 10.1. Let $\mathscr{P}$ be a $\mathrm{TP}_{2}$ temporally homogeneous irreducible Markoff chain whose state space is the non-negative integers. Suppose $\mathscr{P}$ is recurrent; then for any four prescribed states, $i, j, k, l, \lim _{n \rightarrow \infty}\left(P_{i j}^{n} / P_{k l}^{n}\right)$ exists, is finite and positive. 
Proof. The fact that $P_{00}^{n+m}$ is $\mathrm{TP}_{2}$ for $n, m \geqq 0$ (Theorem 2.7, part (i)) yields that $P_{00}^{n+1} / P_{00}^{n}$ is an increasing sequence. Since $\sum_{n=0}^{\infty} P_{00}^{n}=\infty$ we immediately deduce that $\lim _{n \rightarrow \infty} P_{00}^{n+1} / P_{00}^{n}=1$. The theorem now follows by a result of Orey [37].

Another question of probabilistic interest concerns the convergence of the series

$$
\sum_{n=0}^{\infty}\left(P_{j j}^{n}-P_{i j}^{n}\right) \text { for all states } i \text { and } j \text {. }
$$

Throughout this discussion we consider only the case of an aperiodic irreducible Markoff chain.

If both $i$ and $j$ are transient states then the convergence (actually absolutely) is trivial. If $i$ and $j$ are positive recurrent then the convergence is absolute. This is quite easy using generating functions and Wiener's Tauberian Theorem. Chung conjectured that the convergence prevails in all circumstances. However, C. Stone (unpublished) recently produced an example of a recurrent null Markoff chain for which the series is not even $(C, \alpha)$ convergent for any $\alpha>0$. On the other hand, we shall prove that under the assumptions of Theorem 10.1, the series (10.5) converges absolutely.

To this end, we review some preliminaries of general interest. The first remark points out the known result that when (10.5) does not converge, then it oscillates boundedly.

I. $0 \leqq \sum_{n=0}^{m}\left(P_{j j}^{n}-P_{i j}^{n}\right) \leqq C(i, j)$ where $C$ is a constant independent of $m$.

Proof. We start with the identity $P_{i j}^{n}=\sum_{k=0}^{n} F_{i j}^{n-k} P_{j j}^{k}$. Summation gives $\sum_{n=0}^{m} P_{i j}^{n} \leqq \sum_{n=0}^{m} P_{j j}^{n}$ since $\sum_{n=1}^{m} F_{i j}^{n} \leqq 1$ which is the left-hand inequality.

Now summing the identity $P_{j j}^{n}-\sum_{k=0}^{n} F_{j i}^{n-k} P_{i j}^{k}={ }_{i} P_{j j}^{n}$ yields

$$
\sum_{n=0}^{m}\left(P_{j j}^{n}-P_{i j}^{n}\right) \leqq \sum_{n=0}^{m} P_{j j}^{n}-\sum_{n=0}^{m} \sum_{k=0}^{n} F_{j i}^{n-k} P_{i j}^{k}=\sum_{n=0}^{m}{ }_{i} P_{j j}^{n}
$$

It is known that always $\sum_{n=0}^{\infty} P_{j j}^{n}<\infty$ (see [3, Chapter 2]). The right-hand inequality of $I$ is now evident.

The set of identities

$$
\begin{aligned}
P_{i j}^{n}=\sum_{v=0}^{n} P_{i i i}^{v} P_{i j}^{n-v}, & P_{j j}^{n}=\sum_{v=0}^{n} P_{j i t}^{v} P_{i j}^{n-v}+{ }_{i} P_{j j}^{n}, \\
{ }_{i} P_{i j}^{n}=\sum_{v=0}^{n} F_{i j}^{n-v}{ }_{i} P_{j j}^{v}, & F_{j i}^{n}=\sum_{i=0}^{n} P_{i j j}^{v} F_{j i}^{n-v}
\end{aligned}
$$

properly combined yields the generating function relation

$$
{ }_{j} F_{j i}(z) \cdot\left[P_{i j}(z)-P_{j j}(z)\right]+\left[P_{j i}(z)-P_{i i}(z)\right]{ }_{i} F_{i j}(z) F_{j i}(z)=-F_{j i}(z)
$$

where 


$$
{ }_{j} F_{j i}(z)=\sum_{n=0}^{\infty}{ }_{j} F_{j i}^{n} z^{n}, \text { etc. }
$$

(Incidentally, from relations (10.7) it follows generally that $\sum_{i} P_{j j}^{n}<\infty$ and $\sum_{i} P_{i j}^{n}<\infty$.)

Let $\pi_{j i}=\sum_{n=0 j}^{\infty} F_{j i}^{n}=$ the probability that state $i$ is reached before state $j$ starting from state $j$. If (10.5) converges, then set $z=1$ in (10.8) and we obtain

$$
\pi_{j i}\left(\sum_{n=0}^{\infty} P_{i j}^{n}-P_{j j}^{n}\right)+\pi_{i j}\left(\sum_{n=0}^{\infty} P_{j i}^{n}-P_{i i}^{n}\right)=-1 .
$$

Pertaining to (10.9) see also [29]. We further know (without any restrictions but that the process is recurrent) that

$$
\lim _{m \rightarrow \infty} \frac{\sum_{n=0}^{m} P_{j i}^{n}}{\sum_{n=0}^{m} P_{j j}^{n}}=\frac{\pi_{j i}}{\pi_{i j}} .
$$

From (10.9) and (10.10) we can compute $\pi_{i j}$ and $\pi_{j i}$; these are fundamental quantities of the Markoff chain. This lends further justification for the study of the convergence problem. Now

THEOREM 10.2. If the conditions of Theorem 10.1 are satisfied then

$$
\sum_{n=0}^{\infty}\left|P_{j j}^{n}-P_{i j}^{n}\right|<\infty, \quad i, j=0,1,2, \cdots .
$$

Proof. Since $P_{i 0}^{n}$ is $\mathrm{TP}_{2}$ (Theorem 2.6) we find that $P_{00}^{n} / P_{i 0}^{n}$ decreases to a limit $c$ which by Theorem 10.1 is positive. Appealing to the classical Doeblin ratio theorem and a simple Abelian argument using the fact that $\sum_{n=0}^{\infty} P_{00}^{n}=\infty$, we infer that $a=1$. Therefore $P_{00}^{n} \geqq P_{i 0}^{n}$ for all $n$. Hence the terms of (10.5) for $j=0$ are non-negative and by virtue of assertion $\mathrm{I}$ we obtain

$$
\sum_{n=0}^{\infty}\left|P_{00}^{n}-P_{i 0}^{n}\right|=\sum\left(P_{00}^{n}-P_{i 0}^{n}\right) \leqq C .
$$

Now,

$$
P_{j i}^{n}-P_{i i}^{n}=\sum_{v=0}^{n}\left(P_{j 0}^{v}-P_{00}^{v}\right)_{0} P_{0 i}^{n-v}+\sum_{v=0}^{n}\left(P_{00}^{v}-P_{i 0}^{v}\right)_{0} P_{0 i}^{n-v}+{ }_{0} P_{j i}^{n}-{ }_{0} P_{i i}^{n} \text {. }
$$

But all the series $\sum_{n=0}^{\infty}{ }_{0} P_{j i}^{n}, \sum_{n=0}^{\infty}{ }_{0} P_{i i}^{n}, \sum_{n=0}^{\infty}{ }_{0} P_{0 i}^{n}$ and (10.12) converge. If we use the familiar property that the convolution of two absolutely convergent series is again an absolutely convergent series, it follows that $\sum_{n=0}^{\infty}\left|P_{j i}^{n}-P_{i i}^{n}\right|<\infty$.

Applications for continuous-time Markoff chains. We now indicate some analogs of the preceding results for the case of Markoff chains in continuous time. We list them omitting their proofs since they involve the same essential ideas. 
Let $\mathscr{P}$ be a continuous-time Markoff chain satisfying the conditions of Theorem 4.3. As pointed out in $\S 4, \mathscr{P}$ is necessarily a birth-and-death process (see paragraph 9 of $\S 8)$ and the transition density possesses an integral representation exhibited in (8.6).

A. Suppose $\mathscr{P}$ is such that $\sum_{j=0}^{\infty} P_{i j}(t)=1$ for all $t>0$ and $i=0,1,2, \cdots$; then $P_{0 j}(t)$ and $P_{j 0}(t)=P_{0 j}(t) / \pi_{j}$, where $j \geqq 0$ and fixed, are unimodal functions in $t$. (Recall that $\pi_{j}=\lambda_{0} \lambda_{1} \cdots \lambda_{j-1} / \mu_{1} \mu_{2} \cdots \mu_{j}$ where $\lambda_{j}$ and $\mu_{j}, j \geqq 0$, designate the birth and death rates, respectively.) This result derives further interest in that generally $P_{i j}(t)$ is not necessarily a unimodal function in $t$ when $i>0$ and $j>0$. In fact, we construct an example where $P_{12}^{\prime}(t)$ has two zeros $\left({ }^{10}\right)$.

Consider a birth-and-death process with four states labelled $0,1,2,3$. The parameters are

$$
\lambda_{0}=\lambda_{2}=1, \quad \lambda_{1}=\alpha, \quad \mu_{1}=\mu_{2}=\mu_{3}=1 \quad\left(\mu_{0}=\lambda_{3}=0\right)
$$

and so $\pi_{0}=\pi_{1}=1 ; \pi_{2}=\pi_{3}=\alpha$. A direct calculation gives

$$
\begin{aligned}
P_{12}(t)=\alpha\left\{\frac{1}{2(1+\alpha)}-\frac{1}{2}\left(\frac{1}{1+\alpha}-\frac{1}{\sqrt{ }\left((1+\alpha)^{2}+4\right)}\right)\right. \\
\quad \times \exp \left(-\frac{(3+\alpha)-\sqrt{ }\left((1+\alpha)^{2}+4\right)}{2} t\right) \\
+\frac{1}{2(1+\alpha)} e^{-2 t}-\frac{1}{2}\left(\frac{1}{1+\alpha}+\frac{1}{\sqrt{ }\left((1+\alpha)^{2}+4\right)}\right) \\
\left.\quad \times \exp \left(-\frac{(3+\alpha)+\sqrt{ }\left((1+\alpha)^{2}+4\right)}{2} t\right)\right\} .
\end{aligned}
$$

Direct examination of this formula reveals that $P_{12}(t)$ increases for large $t$ for all $\alpha$ and $P_{12}(t) \rightarrow \frac{1}{2}\left(1+e^{-2 t}\right)$ for $t>0$ as $\alpha \rightarrow \infty$. Hence, for $\alpha$ sufficiently large $P_{12}^{\prime}(t)$ necessarily vanishes twice.

B. Suppose $\sum_{j=0}^{\infty} P_{i j}(t)=1$ for all $t \geqq 0$; then $P_{i j}(t)$ unimodal as a function of $i$. Under the same hypothesis, on account of the identity $P_{i j}(t) / \pi_{j}=P_{j i}(t) / \pi_{i}$, we see that $P_{i j}(t) / \pi_{j}$ is unimodal as a function of $j$.

Let $q(t ; i, j)$ denote the density function of the random variable $T_{i j}=$ the time of first passage from state $i$ to state $j$. (Cf. Theorem 4.1.)

C. $q(t ; 0, j), q(t ; i, i+1)$ and $q(t ; i+1, i)$ are each unimodal functions of $t$.

D. If recurrence to the zero state is a certain event, then $q(t ; i 0)$ is a unimodal function of $i$.

We conclude this part by making some observations concerning the nature of the convergence of $P_{i j}(t)$ to its limit as $t$ tends to $\infty$. For this purpose we need to examine the quantity $\lim _{t \rightarrow \infty} P_{i j}^{\prime}(t) / P_{00}^{\prime}(t)$. This involves the study of a ratio of derivatives of the transition matrix comparable to the investigation of ratios

(10) This example is due to W. Pruitt. 
of transition probability functions. A strong ratio theorem for birth-and-death processes was proved in [17]. The same method carries over and we deduce that $\lim _{n \rightarrow \infty} P_{i j}^{\prime}(t) / P_{00}^{\prime}(t)$ exists and is finite.

We now distinguish three cases. Let $\psi$ denote the spectral measure associated with the process $\mathscr{P}$ (see equation (8.6)). Consider the measure $d \psi^{*}=x d \psi(x) / \lambda_{0}$.

Case 1. If $\psi^{*}$ increases in the neighborhood of the origin it follows that

$$
\lim _{t \rightarrow \infty} \frac{P_{i j}^{\prime}(t)}{P_{00}^{\prime}(t)}=\lim _{t \rightarrow \infty} \frac{P_{i j}(t)}{P_{00}(t)}=a_{i j}>0
$$

In this case $a_{i j}=\pi_{j}>0$. Inspection of the integral representation (8.6) clearly reveals that $P_{00}^{\prime}(t)<0$ for all $t$. Thus, in view of $(10.13)$, we have $P_{i j}^{\prime}(t)<0$ for $t$ large.

Case 2. If $\psi$ has no measure at the origin, we secure (10.13) with the limit value $a_{i j}=\pi_{j} Q_{i}(a) Q_{j}(a)$ where $a>0$ is the first positive point in the spectrum of $\psi$. Our normalization condition (see equation (8.7)) implies that $Q_{k}(a)>0$ for all $k$. Hence, as before, we deduce that $P_{i j}^{\prime}(t)<0$ for large $t$.

Case 3. Suppose $\psi$ has an isolated jump at 0 and the next point in the spectrum is located at $b>0$. We are automatically in the ergodic case [17] and $\lim _{t \rightarrow \infty} P_{i j}(t)=\pi_{j} / \sum_{k=0}^{\infty} \pi_{k}>0$. The subsequent analysis reveals how the limit distribution is approached. The methods of [17] yield

$$
\lim _{t \rightarrow \infty} \frac{P_{i j}^{\prime}(t)}{P_{00}^{\prime}(t)}=\pi_{j} Q_{i}(b) Q_{j}(b)
$$

while

$$
\lim _{t \rightarrow \infty} \frac{P_{i j}(t)}{P_{00}(t)}=\pi_{j} .
$$

A familiar argument for orthogonal polynomials shows that there exists $0<k_{0}<\infty$ such that $Q_{k}(b)>0$ for $k<k_{0}$ and $Q_{k}(b)<0$ for $k>k_{0}$. In exceptional situations it is possible that $Q_{k_{0}}(b)=0$, but then $Q_{k_{0}}(b+\varepsilon)<0$ for $\varepsilon>0$ and sufficiently small.

Since always $P_{00}^{\prime}(t)<0$ we sum up as follows: If either $\max (i, j)<k_{0}$ or $\min (i, j) \geqq k_{0}$ then $P_{i j}^{\prime}(t)<0$ for $t$ sufficiently large. If either $i<k_{0}, j \geqq k_{0}$ or $i \geqq k_{0}, j<k_{0}$ then $P_{i j}^{\prime}(t)>0$ for $t$ sufficiently large. In particular, if $i=0$ and $j>k_{0}$ we obtain the interesting feature that $P_{0 j}^{\prime}(t)>0$ everywhere. This is a plain consequence of the facts that $P_{0 j}(t)$ is unimodal, $P_{0 j}(0)=0$, and by what was just proved, namely that $P_{0 j}^{\prime}(t)>0$ for $t$ large. This case arises in the classical example of the telephone trunking process or the model of linear growth where $\lambda_{n}=n \lambda+a$, $\mu_{n}=n \mu, n \geqq 0(\lambda>\mu>0, a>0)$.

Continuous-state Markoff processes. Analogs of the preceding result in the case of general continuous-state Markoff processes are also available. Let $\mathscr{P}$ 
represent a process satisfying the conditions of Theorem 5.2. We record as illustrative two results corresponding to Propositions 6 and 7 of this section.

I. The transition density $p(t ; 0, x)$ is a unimodal function of $t$ for fixed $x>0$ provided $\int p(t, 0, x) d \mu(x)=1$ for all $t>0$.

II. The density function of the first-passage time from 0 to $x q(t ; 0, x)$ is a unimodal function of $t$.

Corresponding results are accesible in the case of discrete-time continuousstate Markoff processes satisfying the hypotheses of Theorem 3.8. We shall give two examples.

1. Consider the density function $f_{\alpha}(x ; \lambda)$ defined in (9.6). It was proved there that for $\alpha>0, f_{\alpha}(x ; \lambda)$ is $\mathrm{PF}_{2}$. By virtue of Proposition 10.1 we conclude that $f_{\alpha}(x ; \lambda)$ is unimodal as a function of $x$. Since $f_{\alpha+\beta}(x ; \lambda)$ is $\mathbf{R R}_{\infty}$ for $\alpha, \beta>0$, we also deduce that $f_{\alpha}(x ; \lambda)$ is unimodal as a function of $\alpha$.

2. In $\$ 9$ we proved that the positive stable law

$$
g(u ; t)=\frac{1}{t^{k}} f_{1 / k}\left(\frac{u}{t^{k}}\right), \quad u>0, t>0,
$$

whose Laplace transform is $e^{-t s^{1 / k}}, k=2,3,4, \cdots$, is $\mathrm{TP}_{\infty}$ for $u>0, t>0$.

We claim that $g(u ; t)$ is unimodal in $u$. More generally, we shall prove that if $K(x, y)=h(x / y)$ is $\mathrm{TP}_{2}$ in the variables $x, y>0$, then $h(u)$ is unimodal. An exponential change of variable $x=e^{\xi}, y=e^{\eta}$ shows that $L(\xi, \eta)=h\left(e^{\xi-\eta}\right)$ is $\mathrm{TP}_{2}$ or in equivalent terms that $h\left(e^{u}\right)=g(u)$ is $\mathrm{PF}_{2}$. By Proposition $1, g(u)$ is unimodal and trivially $h(\xi)$ inherits the same property. We can plainly generalize the result just proved for $h(x / y)$ to the case of a function which has the form $h(\phi(x) / \psi(y))$ where $\phi(x)$ and $\psi(y)$ are strictly monotone increasing and such that $K(x, y)=h(\phi(x) / \psi(y))$ is $\mathrm{TP}_{2}$. The identical argument with trivial modifications persists and we deduce again that $h(u)$ is unimodal. As a special application we see that (10.15) is unimodal as a function of $u>0$.

Other regularity properties. Until now we devoted our studies mainly to the property of unimodality. We indicate briefly other regularity properties implied by total positivity. To this end we offer two applications of Theorem 7.2.

1. Let

$$
c(t)=\int_{0}^{\infty} \frac{\xi^{t-1}}{\Gamma(t)} f(\xi) d \xi, \quad t>0,
$$

where $f(\xi)$ is $\mathrm{PF}_{2}$. The kernel

$$
\phi(t, \xi)= \begin{cases}\frac{\xi^{t-1}}{\Gamma(t)}, & \xi>0, \\ 0, & \xi \leqq 0,\end{cases}
$$

satisfied the conditions of Theorem 7.2. Thus we infer from that theorem that 
$c(t+s)$ is $\mathrm{RR}_{2}$ for $t, s>0$. Manipulating the second order determinantal inequalities satisfied by $c(t+s)$ we achieve the special moment inequalities

$$
\left(\frac{1}{\Gamma(t+1)} \int_{0}^{\infty} \xi^{t} f(\xi) d \xi\right)^{1 / t} \geqq\left(\frac{1}{\Gamma(s+1)} \int_{0}^{\infty} \xi^{s} f(\xi) d \xi\right)^{1 / s}
$$

for $0<t<s$. This derives further interest by comparison with the classical moment inequality

$$
\left(\int_{0}^{\infty} \xi^{t} f(\xi) d \xi\right)^{1 / t} \leqq\left(\int_{0}^{\infty} \xi^{s} f(\xi) d \xi\right)^{1 / s}
$$

for $0<t<s$ which is in the opposite direction.

We also deduce the interesting property by invoking Proposition 1 of this section that $c(t)$ is unimodal.

2. Consider

$$
c_{n}(x)=\sum_{k=0}^{n}\left(\begin{array}{l}
n \\
k
\end{array}\right) \frac{x^{k}}{\Gamma(\alpha+k+1)}, \quad \alpha>0 .
$$

The identity

$$
\left(\begin{array}{c}
m+n \\
k
\end{array}\right)=\sum_{l=0}^{\infty}\left(\begin{array}{c}
m \\
l
\end{array}\right)\left(\begin{array}{c}
n \\
k-l
\end{array}\right)
$$

corresponds to (7.7). Also $\left(\begin{array}{l}n \\ k\end{array}\right)$ is $\mathrm{TP}_{\infty}$ as witnessed in example 1 of $\S 8$. Furthermore by Lemma 9.1,

$$
\frac{x^{k+l}}{\Gamma(\alpha+k+l+1)}=f_{k+l}
$$

is $\mathbf{R}_{\infty}$ for $k, l \geqq 0$ since $\alpha>0$.

Applying Theorem 7.2 we conclude that

$$
c_{n+m}(x)=\frac{Q_{n+m}^{\alpha}(-x)}{\Gamma(\alpha+1)},
$$$$
x>0
$$

is $\mathbf{R} \mathbf{R}_{\infty}$ in the variables $n, m \geqq 0$. (Here, $Q_{k}^{\alpha}(x)$ agrees with the classical Laguerre polynomials of order $\alpha$ normalized so that $Q_{k}^{\alpha}(0)=1$ [34].) In contrast, it is easy to prove that $\Gamma(n+m+\alpha+1) Q_{n+m}^{\alpha}(-x)=R(n+m)$ is $\mathrm{TP}_{\infty}$ for $n, m \geqq 0$ (see [26]).

11. The absolute value process and related totally positive processes. In this section we indicate several examples of stochastic processes of some interest whose transition probability function is totally positive to which the theory of the preceding sections is applicable.

In the first four examples $\mathscr{P}$ represents a temporally homogeneous Markoff chain, whose state space is the non-negative integers with transition matrix $P_{i j}$. 
1.

$$
P_{i j}= \begin{cases}a_{j}, & i=0,1,2, \cdots, k_{0}, \\ a_{j-i+k_{0}}, & i>k_{0}+1,\end{cases}
$$

where $a_{j}(j=0,1,2, \cdots)$ is a $\mathrm{PF}_{r}$ probability sequence. A direct verification shows that $\mathscr{P}$ is $\mathrm{TP}_{r}$. Markoff chains of the type (11.1) arise in the theory of queues.

Consider the case $k_{0}=1$ and let

$$
a_{j}=\int_{0}^{\alpha} \frac{\xi^{j} e^{-\xi} f(\xi)}{\Gamma(j+1)} d \xi, \quad j=0,1,2, .
$$

This corresponds to a queueing process whose interarrival time of successive customers is a random variable following an exponential distribution and whose service time random variable has the density function $f(\xi)$. The quantity $a_{j}$ is the probability that $j$ customers arrive during a service period.

Now, if $f(\xi-\eta)$ is $\mathrm{TP}_{2}$ for $-\infty<\xi, \eta<\infty$, then $e^{-\xi} f(\xi)$ is also $\mathrm{PF}_{2}$ (see Lemma 9.2). This is satisfied if for example $f(\xi)=\xi^{\alpha} e^{-\xi} / \Gamma(\alpha+1), \alpha>0$ (see (9.13)). According to Theorem $7.2, \mathfrak{l}_{j+k}$ is $\mathrm{RR}_{2}(j, k \geqq 0)$ and consequently $a_{j}$ is $\mathrm{PF}_{2}$.

2.

$$
P_{i j}=a_{\min (i, j)} b_{\max (i, j)}, \quad \quad i, j=0,1, \cdots .
$$

If $a_{i}, b_{i}>0$ for all $i$ and $b_{i} / a_{i}$ is decreasing we obtain that $\mathscr{P}$ is $\operatorname{TP}_{\infty}$. (See Lemma 11.1 below.)

3.

$$
\begin{aligned}
& P_{i j}=\left\{\begin{array}{ll}
a, & j=i+1, \\
b, & j=i, \\
c, & j=i-1, \\
0, & \text { otherwise. }
\end{array} \quad i=1,2, \cdots,\right. \\
& P_{00}=b+c, \quad P_{01}=c .
\end{aligned}
$$

If $b \geqq 2 \sqrt{ }(a c)$, then $\mathscr{P}$ is $\mathrm{TP}_{\infty}$. This example was studied in detail in $\S 10$, paragraph 6 .

4. Let

$$
P_{\imath j}=\left(\begin{array}{c}
N \\
j
\end{array}\right)\left(\frac{i}{N}\right)^{j}\left(1-\frac{i}{N}\right)^{N-j}, \quad i, j=0,1, \cdots, N
$$

Since $\exp (j \log i /(N-i))$ is $\operatorname{TP}_{\infty}$ for $0<j<N$ and $0<i<N$ we infer that $\mathscr{P}$ is $\mathrm{TP}_{\infty}$. This example arises in the study of certain genetic models.

The absolute value process. We now describe a general construction of a continuous-state discrete-time $\mathrm{TP}_{\text {o. }}$ Markoff chain associated with a symmetric Pólya function.

Let $X$ be a real-valued symmetric random variable with density function $f(x)$. 
For each fixed $u>0$ let $f^{*}(v ; u)$ denote the density function of the random variable $|u+X|$. The random variable $|u+X|$ with $u>0$ serving as a parameter will be referred to as the generalized absolute value (G. A. V.) associated with $X$. Clearly, $f^{*}(v ; u)=f(-u-v)+f(-u+v)$.

Let $Y$ also represent a real-valued symmetric random variable with density function $g(y)$ and suppose $g^{*}(v ; u)$ corresponds to $g$ in the same way that $f^{*}$ corresponds to $f$. Consider now $X+Y=Z$ where $X$ and $Y$ are independent random variables; $Z$ is obviously symmetric.

The absolute value variable $|u+Z|$ associated with $Z$ has a density function $h^{*}(v ; u)$ which is obtained from $f^{*}$ and $g^{*}$ by a generalized convolution operation. Explicitly

$$
h^{*}(v ; u)=\int_{0}^{\infty} f^{*}(v ; w) g^{*}(w ; u) d w .
$$

The proof is simple; consider

$$
\begin{aligned}
h^{*}(v ; u)= & \operatorname{Pr}\{|u+X+Y|=v\} \\
= & \int_{0}^{\infty} \operatorname{Pr}\{|u+X+Y|=v \mid u+Y=w\} g(w-u) d w \\
& \quad+\int_{0}^{\infty} \operatorname{Pr}\{|u+X+Y|=v \mid u+Y=-w\} g(-w-u) d w \\
= & \int_{0}^{\infty} \operatorname{Pr}\{|w+X|=v\} g^{*}(w ; u) d w,
\end{aligned}
$$

the last resulting on account of the symmetry of $X$ coupled with the fact that $X$ is independent of $Y$.

It follows from (11.5) by virtue of $(0.4)$ that if $f^{*}(v ; w)$ and $g^{*}(w ; u)$ are $\operatorname{TP}_{r}$ then $h^{*}(v ; u)$ is also $\mathrm{TP}_{r}$. Thus the class of symmetric random variables for which the corresponding G.A.V. is $\mathrm{TP}_{r}$ is closed with respect to the formation of sums of independent variables.

This class includes the normal distribution of mean zero and arbitrary variance. To prove this we use the fact that the Wiener process $\xi(t)$ is a strong Markoff process with almost all continuous sample path. Hence the absolute value process $|\xi(t)|=\eta(t)$ likewise determines a strong Markoff process with continuous sample paths. Now appealing to Theorem 1 in [20] (see also paragraph 11 of §8) we conclude that the transition density function of the $\eta(t)$ process is for each fixed $t, \mathrm{TP}_{\infty}$ with respect to the initial-and-final state variables. This result for $t=\sigma^{2}$ reduces to the statement that the density $f^{*}(v ; u)$ of a G.A.V. corresponding to an underlying normal distribution $N\left(0, \sigma^{2}\right)$ is $\mathrm{TP}_{\infty}$.

The random variables with density function $g(x)=(c / 2) \exp (-c|x|)(c>0)$ 
induces a family of density functions $g^{*}(v ; u)$ which is $\mathbf{T P}_{\infty}$. In order to prove this assertion we quote the following

LEMMA 11.1. For $u, v \geqq 0$, let

$$
K(u, v)= \begin{cases}f_{1}(u) f_{2}(v), & u \leqq v, \\ f_{1}(v) f_{2}(u), & u \geqq v,\end{cases}
$$

where $f_{1}$ and $f_{2}$ are positive functions. If $f_{1}(\xi) / f_{2}(\xi)$ is monotone increasing, then $K(u, v)$ is $\mathrm{TP}_{\infty}$.

For the proof of this lemma the reader may consult [9, Chapter 2]. A direct verification is also not difficult.

The function $g^{*}(v ; u)$ corresponding to $g(x)=(c / 2) \exp (-c|x|)$ exhibits the form (11.6). In fact,

$$
\begin{aligned}
g^{*}(v ; u) & =\frac{c}{2}\{(\exp (-c|-u-v|)+\exp (-c|-u+v|))\} \\
& = \begin{cases}\frac{c}{2}\left(e^{-c u}+e^{c u}\right) e^{-v c}, & u<v, \\
\frac{c}{2}\left(e^{-c v}+e^{c v}\right) e^{-c u}, & u>v,\end{cases}
\end{aligned}
$$

and

$$
\frac{f_{1}(\xi)}{f_{2}(\xi)}=\frac{e^{-c \xi}+e^{c \xi}}{e^{-c \xi}}=1+e^{2 c \xi}
$$

is clearly monotone increasing. Applying Lemma 11.1 we conclude that (11.7) is $\mathrm{TP}_{\infty}$.

We are now in a position to prove the following theorem (this result and Theorem 11.2 are due jointly to $\mathrm{C}$. Stone and this writer):

THEOREM 11.1. Let $X$ be a random variable whose density function $f(x)$ is a symmetric Pólya frequency function $\mathrm{PF}_{\infty}$. Then the density function $f^{*}(x ; u)$ of the random variable $|X+u|(u>0$ is a parameter $)$ is $\mathbf{T P}_{\infty}$.

Proof. The representation theorem for Pólya frequency densities states that $f(x)$ is such a density if and only if its Laplace transform $\phi(s)$ exists in a strip including the imaginary axis on its interior and has the form

$$
\phi(s)=\frac{e^{-\mu s^{2}+v s}}{\prod_{i=1}^{\infty}\left(1+\frac{s}{a_{\imath}}\right) e^{-s / a_{i}}}
$$

where $\mu \geqq 0, v, a_{i}$ are real and $\sum_{i=1}^{\infty} 1 / a_{i}^{2}<\infty[33]$. If $f(x)$ is symmetric then (11.8) reduces to 


$$
\phi(s)=\frac{e^{-\mu s^{2}}}{\prod_{i=1}^{\infty}\left(1-\frac{s^{2}}{\lambda_{i}^{2}}\right)},
$$$$
\mu \geqq 0, \sum_{i=1}^{\infty} \frac{1}{\lambda_{i}^{2}}<\infty,
$$

and $\lambda_{i}$ are real and nonzero. The special Pólya frequency density $g(t)$ $=(c / 2) \exp (-|t| c)$ has the Laplace transform $1 /\left[1-\left(s^{2} / c^{2}\right)\right]$ which is (11.9) with a single factor. According to the preceding analysis $g^{*}(v ; u)$ corresponding to this density is $\mathrm{TP}_{\infty}$. The density function $g_{n}(\xi)$ whose Laplace transform is

$$
\phi(s)=\frac{1}{\prod_{i=1}^{n}\left(1-\frac{s^{2}}{\lambda_{i}^{2}}\right)},
$$

is represented as a sum of $n$ independent variables each with density function $(c / 2) \exp (-|t| c)$ and suitable choices of $c$. The convolution formula (11.5) implies that $g_{n}^{*}(v ; u)$ corresponding to $g_{n}(\xi)$ is $\mathrm{TP}_{\infty}$. Since $\phi_{n}(s)$ converges to

$$
\frac{1}{\prod_{i=1}^{\infty}\left(1-\frac{s^{2}}{\lambda_{i}^{2}}\right)}=\phi^{*}(s)
$$

uniformly on the whole imaginary axis, we find by inverting the Laplace transform and using the principle of bounded convergence that $g_{n}(\xi)$ converges uniformly to $h(\xi)$, the density function whose Laplace transform is $\phi^{*}(s)$. It follows that $h^{*}(v ; u)$ is $\operatorname{TP}_{\infty}$.

Moreover, we already established that the normal law (Laplace transform $e^{-\mu s^{2}}$ ) has a G.A.V. density which is $\mathrm{TP}_{\infty}$. Again appealing to the convolution formula (11.5) we deduce in general that if $f(v)$ represents the density function whose Laplace transform is $(11.9)$ then $f^{*}(v ; u)$ is $\mathrm{TP}_{\infty}$. The proof of the theorem is complete.

We may develop a discrete analog of Theorem 11.1. Let $c_{v}(v=0, \pm 1, \pm 2, \cdots)$ denote a $\mathrm{PF}_{\infty}$ sequence for which $\sum_{v=-\infty}^{\infty} c_{v}=1$. The Laurent series generating function $f(z)=\sum_{v=-\infty}^{\infty} c_{v} z^{v}$ admits a representation

$$
F(z)=\exp \left(a z+b z^{-1}\right) \frac{\prod_{i=1}^{\infty}\left(1+\alpha_{i} z\right) \prod_{i=1}^{\infty}\left(1+\beta_{i} z^{-1}\right)}{\prod_{i=1}^{\infty}\left(1-\gamma_{i} z\right) \prod_{i=1}^{\infty}\left(1-\delta_{i} z^{-1}\right)}
$$

which is meromorphic in the ring including the unit circle on its interior and $a \geqq 0, b \geqq 0, \alpha_{i} \geqq 0, \beta_{i} \geqq 0,0 \leqq \gamma_{i}<1,0 \leqq \delta_{i}<1, \quad \Sigma_{i}\left(\alpha_{i}+\beta_{i}+\gamma_{i}+\delta_{i}\right)<\infty[6]$. Conversely any function of the form (11.10) when expanded in a Laurent series has the property that the coefficients are a $\mathrm{PF}_{\infty}$ sequence. If $\left(c_{v}\right)$ is symmetric (i.e., $c_{v}=c_{-v}$ ) then $(11.10)$ reduces to 


$$
F(z)=\exp \left(a\left(z+\frac{1}{z}\right)\right) \frac{\prod_{i=1}^{\infty}\left(\left(1+\alpha_{i}^{2}\right)+\alpha_{i}\left(z+\frac{1}{z}\right)\right)}{\prod_{i=1}^{\infty}\left(1-\gamma_{i} z\right)\left(1-\gamma_{i} z^{-1}\right)} .
$$

Now let $\xi$ define a symmetric random variable with probability distribution $\operatorname{Pr}\{\xi=k\}=c_{k}(k=0, \pm 1, \pm 2, \cdots)$.

We associate with $\xi$ the absolute value random variable $|n+\xi|$ whose density function is denoted by $f(k ; n)$ where $n$ serves as a parameter, $n \geqq 0$ and integral. We consider some important examples.

I. Let $\xi$ represent a random variable such that $\operatorname{Pr}\{\xi=0\}=\left(1+\alpha^{2}\right) /(1+\alpha)^{2}$, $\operatorname{Pr}\{\xi=1\}=\operatorname{Pr}\{\xi=-1\}=\alpha /(1+\alpha)^{2}$. Its generating function is $\left[(1+\alpha)^{2}\right]^{-1}\left[\left(1+\alpha^{2}\right)+\alpha(z+1 / z)\right]$ which apart from a constant multiplier corresponds to a single factor of the numerator in (11.11). In this case the density function $f(k ; n)$ apart from a common factor $\left[(1+\alpha)^{2}\right]^{-1}$ in each row can be exhibited as the matrix:

$$
A=\left[\begin{array}{cccccc}
1+\alpha^{2} & 2 \alpha & 0 & 0 & 0 & \ldots \\
\alpha & 1+\alpha^{2} & \alpha & 0 & 0 & \ldots \\
0 & \alpha & 1+\alpha^{2} & \alpha & 0 & \ldots \\
\vdots & \vdots & \vdots & \vdots & &
\end{array}\right]
$$

This matrix is of the form of example 3 of this section except for the first row (see also paragraph 6, §8). The method employed there works in this case as well and we conclude the $\mathrm{TP}_{\infty}$ property.

II. Let $\xi$ represent a random variable for which $\operatorname{Pr}\{\xi=v\}=c_{v}$ where $c_{0}=2 c, c_{v}=c e^{-a|v|}, v= \pm 1, \pm 2, \cdots, a>0$, and $c=\left(1-e^{-a}\right)^{2}$ is a normalizing constant.

Its generating function is of the form

$$
\frac{(1-\gamma)^{2}}{(1-\gamma z)\left(1-\gamma z^{-1}\right)}
$$$$
\gamma=e^{-a}
$$

which corresponds to a single factor of the denominator in (11.11).

Adapting the argument following Lemma 11.1 we deduce easily for this case that $f(k ; n)=\operatorname{Pr}\{|\xi+n|=k\}, k, n=0,1, \cdots$, is $\operatorname{TP}_{\infty}$.

Observe that

$$
\exp \left[a\left(z+\frac{1}{z}\right)\right]=\lim _{n \rightarrow \infty}\left(1+\frac{a z}{n}\right)^{n}\left(1+\frac{a}{z n}\right)^{n} .
$$

Now combining the terms of (11.11) in the fashion of the proof of Theorem 11.1 
we arrive at the general result to the effect that $f(k ; n)=\operatorname{Pr}\{|\xi+n|=k\}$ is $\mathrm{TP}_{\infty}$ under the condition that $\xi$ is a symmetrical $\mathrm{PF}_{\infty}$ sequence. We omit the formal details. To sum up,

THEOREM 11.2. Let $\xi$ be a random variable whose density is a symmetric Pólya frequency sequence. Then the density function $f(k ; n)$ of the random variable $|\xi+n|\left(n \geqq 0\right.$ a non-negative integer) is $\operatorname{TP}_{\infty}$ in the variables $n, k \geqq 0$.

As an illustration we find that $f(k ; n)=q^{(n+k)^{2}}+q^{(n-k)^{2}}$ is $\mathbf{T P}_{\infty}$ in the variables $k, n \geqq 0$. Here, $0<q<1$.

Theorem 11.1 and Theorem 11.2 combined with Theorem 3.8 and Theorem 2.6 respectively yield the following interesting results:

(i) Let $X_{i}, i=1,2, \cdots$, represent independent identically distributed random variables satisfying the hypothesis of Theorem 11.1. Then the function

$$
Q(m, y)=\operatorname{Pr}\left\{\left|X_{1}+X_{2}+\cdots+X_{m}\right|<y\right\}
$$

is $\mathrm{TP}_{\infty}$ in the variables $m \geqq 1$ and $y>0$.

(ii) Let $\xi_{i}, i=1,2, \cdots$, represent independent and identically distributed random variables satisfying the hypothesis of Theorem 11.2. Then the function

$$
Q(m, k)=\operatorname{Pr}\left\{\left|\xi_{1}+\xi_{2}+\cdots+\xi_{m}\right|=k\right\}
$$

is $\mathrm{TP}_{\infty}$ in the variables $m \geqq 0, k \geqq 0$ provided $Q(0 . k)=\delta_{0 k}$ by definition.

\section{BIBLIOGRAPHY}

1. G. Baxter and M. Donsker, On the supremum functional for processes with stationary independent increments, Trans. Amer. Math. Soc. 85 (1957), 73-88.

2. S. Bochner, Harmonic analyses and the theory of probability, Univ. of California Press, Berkeley, Calif., 1955.

3. K. L. Chung, Markoff chains, Springer, Berlin, 1960.

4. D. A. Darling and A. J. F. Siegart, The first passage problem for a continuous Markoff process, Ann. Math. Statist. 24 (1953), 624-639.

5. E. B. Dynkin, One dimensional continuous strong Markov processes, Theory of Probability and its Applications 4 (1959), 1-53. (Russian)

6. A. Edrei, On the generating functions of totally positive sequences, J. Analyse Math. 2 (1952), 104-109.

7. W. Feller, Diffusion processes in one dimension, Trans. Amer. Math. Soc. 77 (1954), 1-31.

8. - - On second order differential operators, Ann. of Math. (2) 61 (1951), 90-105.

9. F. Gantmacher and M. Krein, Oscillation matrices and vibrations of mechanical systems, Moscow, 1950.

10. W. Hahn, Uber Orthogonalpolynome, die p-differenzgleichungen genugen, Math. Nachr. 2 (1949), 4-34.

11. I. I. Hirschman, Hankel transforms and variation diminishing kernels, Bull. Amer. Math. Soc. 66 (1960), 40-42.

12. I. I. Hirschman and D. V. Widder, The convolution transform, Princeton Univ. Press, Princeton, N. J., 1955. 
13. S. Karlin, Decision theory for Pólya type distributions, case of two actions. I, Proc. Third Berkeley Symposium on Probability and Statistics, Vol. 1, pp. 115-129. Univ. of California Press, Berkeley, Calif., 1956.

14. - Pólya type distributions. II, Ann. Math. Statist. 28 (1957), 281-308.

15. - Total positivity and applications to probability and statistics, Stanford Univ. Press, Stanford, Calif., (to appear).

16. S. Karlin and J. L. McGregor, The differential equations of birth and death processes and the Stieltjes moment problem, Trans. Amer. Math. Soc. 85 (1957), 489-546.

17. - - The classification of birth and death processes, Trans. Amer. Math. Soc. 86 (1957), 366-401.

18. — Classical diffusion processes and total positivity, J. Math. Anal. Appl. 1 (1960) 163-183.

19. — Random walks, Illinois J. Math. 3 (1959), 66-81.

20. —- Coincidence probabilities, Pacific J. Math. 9 (1959), 1141-1164.

21. - Coincidence properties of birth and death processes, Pacific J. Math. 9 (1959), $1109-1140$.

22. - A characterization of the birth and death processes, Proc. Nat. Acad. Sci. U. S. A. 45 (1959), 375-379.

23. - Total positivity of fundamental solutions of parabolic equations, Proc. Amer. Math. Soc. 13 (1962), 136-139.

24. S. Karlin and F. Proschan, Pólya type distributions of convolutions, Ann. Math. Statist. 31 (1960), 721-736.

25. S. Karlin and $\mathrm{H}$. Rubin, The theory of decision procedures for distributions with monotone ikelihood ratio, Ann. Math. Statist. 27 (1956), 272-299.

26. S. Karlin and G. Szegö, Determinant properties of certain orthogonal polynomials, J. Analyse Math. (1961).

27. P. Levy, Processus stochastiques et mouvement Brownian, Gauthier-Villars, Paris, 1948.

28. H. P. McKean, Elementary solutions for certain parabolic partial differential equations, Trans. Amer. Math. Soc. 82 (1956), 519-548.

29. S. Orey, Sums arising in the theory of Markoff chains, Univ. of Minnesota, Tech. Rep. 3, 1961.

30. G. Pólya and G. Szegö, Aufgaben und Lehrasatze aus der Analysis, Vols. I, II, Berlin, 1925.

31. G. Black and F. Proschan, On optimal redundancy, Operations Res. 7 (1959), 581-588.

32. I. J. Schoenberg, On smoothing operations and their generating functions, Bull. Amer. Math. Soc. 59 (1953), 199-230.

33. — On Pólya frequency functions, J. Analyse Math. 1 (1951), 331-374.

34. G. Szegö, Orthogonal polynomials, Amer. Math. Soc. Colloq. Publ. Vol. 23, Amer. Math. Soz., Providence, R. I., 1939.

35 K. L. Chung and P. Erdös, Probability limit theorems assuming only the first moment. I, Mem. Amer. Math. Soc. No. 6 (1951), 19 pp.

36. G. Pólya, Festschrift, Stanford Univ. Press, Stanford, Calif., 1962.

37. S. Orey, Strong ratio limit property, Bull. Amer. Math. Soc. 67 (1961), 571.

STANFORD UNIVERSITY, Stanford, CALIFornia 